\title{
Salford State of the Voluntary, Community and Social Enterprise Sector 2017
}

\section{$A$ report on social and economic impact}

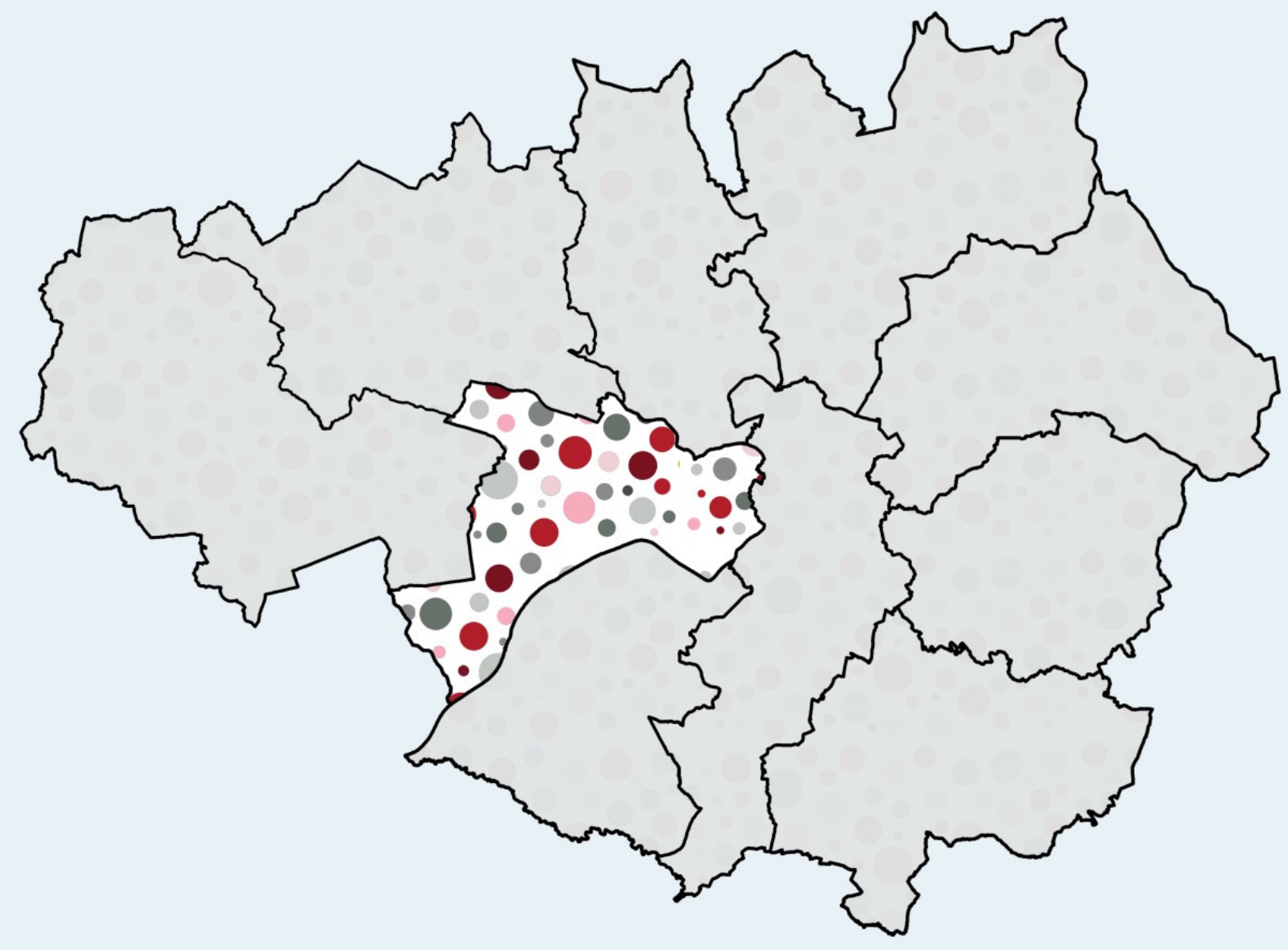

Sheffield Hallam University
Centre for

Regional Economic 


\section{Salford State of the Voluntary, Community and Social Enterprise Sector 2017}

Centre for Regional Economic and Social Research

Sheffield Hallam University

Chris Damm

loannis Prinos

Elizabeth Sanderson

DOI: $10.7190 /$ cresr.2017.6344484429

June 2017 


\section{Acknowledgements}

This research has been commissioned by Salford CVS as part of $10 \mathrm{GM}^{1}$ with GMCVO and undertaken by the Centre for Regional Economic and Social Research (CRESR) at Sheffield Hallam University. This Salford report forms part of a wider collection of reports: there is a Greater Manchester wide report and a report for each of the other local authority areas participating in the study ${ }^{2}$. The full collection of reports can be downloaded from the CRESR website (http://www.shu.ac.uk/research/cresr/reports) and the websites of the project partners.

In completing the report we are particularly grateful to members of the Research Steering Group ${ }^{3}$ and the Salford CVS team for their support in developing and administering the survey and for their input into earlier drafts of the report. We are also grateful to the many employees and volunteers from across the voluntary, community and social enterprise sector who took the time to complete a questionnaire or participate in a focus group.

\section{Contact information}

For CRESR:

$\begin{array}{ll}\text { Name: } & \begin{array}{l}\text { Elizabeth Sanderson } \\ \text { Address: }\end{array} \\ & \text { Research Fellow } \\ & \text { Unit 10 Science Park } \\ & \text { City Campus } \\ & \text { Howard Street } \\ & \text { Sheffield } \\ & \text { S11WB } \\ \text { Tel: } & 01142254814 \\ \text { Email: } & \text { e.sanderson@shu.ac.uk }\end{array}$

For Salford CVS:

\begin{tabular}{|c|c|}
\hline Name: & Marie Wilson \\
\hline & Partnerships and Consortia Manager \\
\hline Address: & $\begin{array}{l}5 \text { Irwell Place } \\
\text { Eccles } \\
\text { Salford } \\
\text { M30 OFN }\end{array}$ \\
\hline $\begin{array}{l}\text { Tel: } \\
\text { Email: }\end{array}$ & 01617877795 \\
\hline & marie.wilson@salfordcvs.co.uk \\
\hline
\end{tabular}

\footnotetext{
${ }^{1} 10 \mathrm{GM}$ is a joint venture by the Greater Manchester Voluntary Sector Infrastructure Organisations (Action Together in Oldham and Tameside, Bolton CVS, CVS Rochdale, Macc, Salford CVS, VCAT, Wigan \& Leigh CVS)

${ }_{2}^{2}$ The other areas are: Bolton, Manchester, Oldham, Rochdale, Stockport, Tameside and Wigan.

${ }^{3}$ The following organisations were represented on the Research Steering Group: CRESR, Salford CVS (lead partner), Greater Manchester Centre for Voluntary Organisation (GMCVO), Bolton CVS, Macc, Action Together in Oldham and Tameside and CVS Rochdale.
} 


\section{Definitions}

This report is about the 'state of the voluntary, community and social enterprise sector in Salford'. At various times the voluntary sector has been known as the 'voluntary and community sector' or the 'third sector' whilst the current Government talks a lot about 'civil society'. In this report, when we talk about the voluntary sector in Salford, we mean voluntary organisations, community groups, the community work of faith groups, and those social enterprises where there is a wider accountability to the public via a board of trustees or a membership and all profits will be reinvested in their social purpose. 


\section{Foreword}

Salford Community and Voluntary Services (Salford CVS) is proud to present this Salford State of the VCSE Sector 2017 report. Following on from similar reports in 2010 and again in 2013 the narrative highlights the social and economic impact of voluntary organisations, community groups and social enterprises in the City of Salford. Salford CVS is pleased that once again we have been able to lead a collaborative approach to this work with partners from other infrastructure support organisations from across Greater Manchester.

Salford CVS' overarching aim is to influence, connect and develop the voluntary, community and social enterprise sector in Salford. Something we have been working hard to do in Salford since 1973! An increasing aspect of Salford CVS' work in recent years has been not just to support individual VCSE organisations but also to help develop strategic partnerships both within and across sectors. One example of this has been the creation of our successful Third Sector Fund, financed by NHS Salford CCG and delivered in partnership with the CCG and Salford City Council, which is making an investment of $£ 1$ million p.a. into the VCSE sector via a number of small grant schemes.

When writing the foreword to this report I had in mind two important mottos that I wanted to highlight:

The welfare of the people is the highest law - from the City of Salford's coat of arms

Deeds Not Words - the motto of the suffragettes' Women's Social and Political Union (WSPU)

These two mottos act as a reminder that the VCSE sector in Salford plays a crucially important role in the civic life of the city - working actively to ensure that the needs and aspirations of Salford citizens are at the forefront of their activities.

This report has been produced at a time when the role of the VCSE sector is becoming increasingly important during this era of immense change, with poverty and inequality rubbing shoulders with significant pockets of growth in Salford, all against the backdrop of a sustained period of austerity, welfare reform, and GM devolution, to name but three influencing factors.

This Salford State of the VCSE Sector 2017 report offers a timely update on the state of the voluntary, community and social enterprise (VCSE) sector in Salford. It outlines the size, diverse scope and amazing breadth of voluntary and community action and activity across the city. It also clearly highlights the significant contribution that the voluntary, community and social enterprise sector makes to the local economy, to people's health and wellbeing, and to local communities themselves. Social value is of course at the heart of VCSE activity in Salford, with VCSE organisations having social aims at the core of their existence. Whilst social value might be a given, the added value that the sector brings to the city is truly impressive.

However, the report also highlights areas for concern, where the sustainability of VCSE organisations is more at risk than ever before, whilst at the same time they struggle to meet the increasing needs of Salford's communities. Income volatility features strongly in this report, which demonstrates that unpredictable funding patterns over the past few years has left many organisations (especially medium-sized organisations) in a position where they now have less than 
three months of running costs in reserve. This is creating growing pressure on the sector and its leadership as it tries to balance the need to income generate alongside the challenge of finding dynamic solutions to address increasing demands on its services.

Understanding our sector is a challenging task. With over 1,500 organisations active across the city, making 2.4 million interventions with beneficiaries in the past year, our scale and reach within communities is unprecedented. This is astounding when you consider that many of these organisations are micro or small, having a turnover of less than $£ 100,000$ per annum. We also continue to have an established culture of volunteering within our city, with over 46,000 people giving their time to benefit others. The research demonstrates that we are a diverse sector, providing a vast range of activities, including 50 per cent of organisations supporting health and wider wellbeing and 44 per cent providing community development work. I'll leave you to explore some more of the highlights in the following executive summary.

This report provides us with a strong incentive to make an urgent call to action. For whilst we currently have a fairly resilient VCSE sector within Salford, despite the challenges of austerity in the context of increasing demands, the picture is also one of fragility. If we are to ensure we have a VCSE sector in Salford that is fit for purpose and equipped to meet increasing need and the looming shift in demand as the public sector transforms, then we need to see continued investment in the VCSE sector by the public sector, including a resurgence in the use of grants (see the Grants for Good campaign at https://www.dsc.org.uk/grantsforgood/ if you aren't convinced!), and a move by more VCSE sector organisations to explore the potential of social investment, working with the private sector, and income generation activities (where feasible of course).

To conclude, I hope that everyone reading this report will value the significant contribution that the VCSE sector makes to the City of Salford. We know that Salford's communities are stronger because of the support and opportunities provided by social enterprises, voluntary organisations, and community groups across Salford. Please share this report with whoever might find it interesting; and come and talk to us at Salford CVS if you would like to find out more about this report or the work we do, both in Salford and alongside partners in Greater Manchester.

Finally, I would like to thank our 10GM partners (fellow infrastructure, support and development organisations in the neighbouring boroughs in Greater Manchester), GMCVO and Sheffield Hallam University for their collaboration in making this happen. I also particularly want to thank all those Salford VCSE organisations who took time to complete the survey, despite other competing pressures for their time. Without you there would be no report!

\title{
Enjoy reading :;
}

\author{
Alison Page \\ Chief Executive \\ Salford CVS
}




\section{Contents}

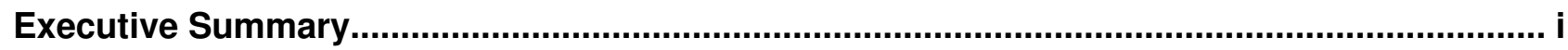

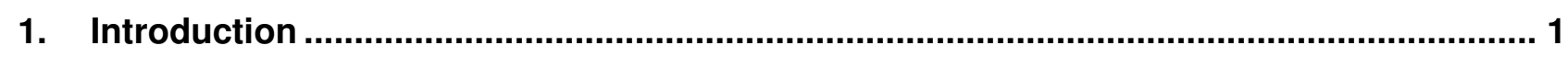

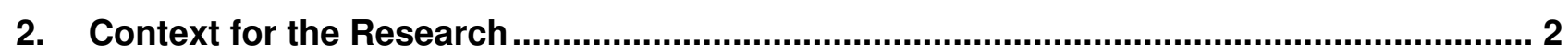

3. What the Voluntary Community and Social Enterprise Sector in Salford does............... 4

4. Who the Voluntary Community and Social Enterprise Sector in Salford works with......11

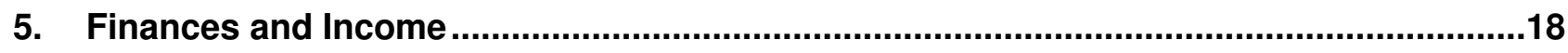

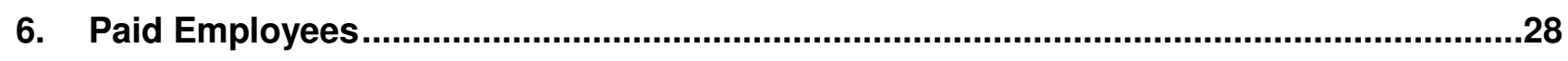

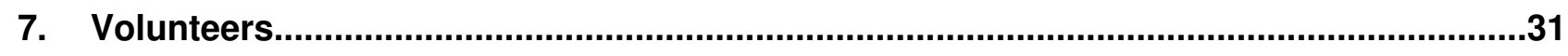

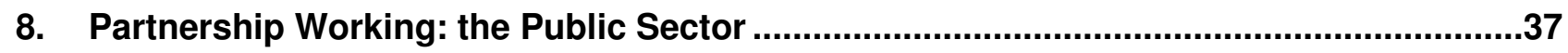

9. Partnership Working: the Private Sector ........................................................................

10. Partnership Working: Voluntary Community and Social Enterprise Organisations........50

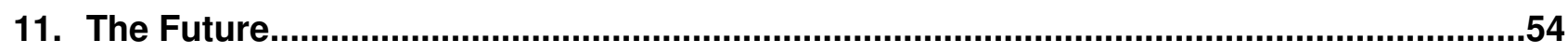

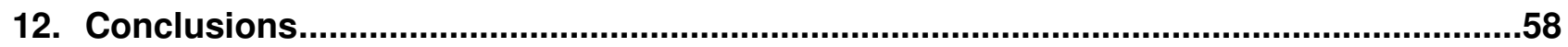

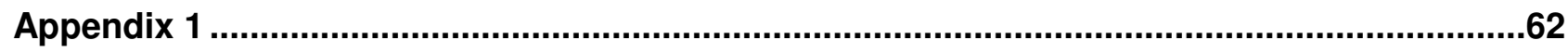




\section{Executive Summary}

This report provides the main findings of research aimed at improving the understanding of the social and economic impact of the voluntary, community and social enterprise (VCSE) sector in Salford. The key objective of the research was to provide a comprehensive overview of the sector in Salford at the start of 2017. Where possible the report compares results from the latest survey and an earlier 2012/13 study. Revisions to the questionnaire and methodology between these studies however mean that comparisons are not always possible or appropriate (see appendix 1).

In this summary we answer eleven key questions about the sector and its role across Salford.

\section{Q1. How many organisations are there?}

There are an estimated $\mathbf{1 , 5 1 3}$ organisations working in the VCSE sector in Salford and the vast majority of organisations are micro or small (87 per cent less than $£ 100,000$ ). This estimate includes formally registered organisations, such as charities, social enterprises and co-operatives, but it also includes a large number of 'below the radar' organisations that are not formally registered or incorporated.

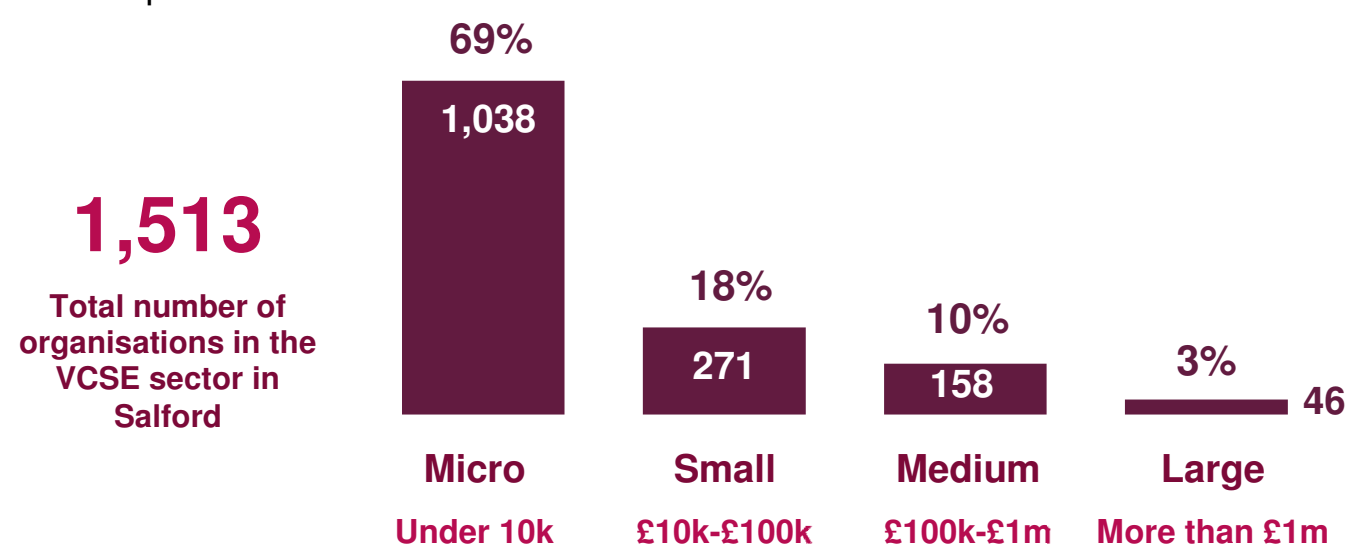

\section{Q2. Who benefits from their work?}

The overall client group is diverse and wide ranging. The client groups served by the largest proportions of organisations can be broadly characterised as being demographic. Just over a quarter of organisations surveyed identified 'everyone' as their main clients, users or beneficiaries.

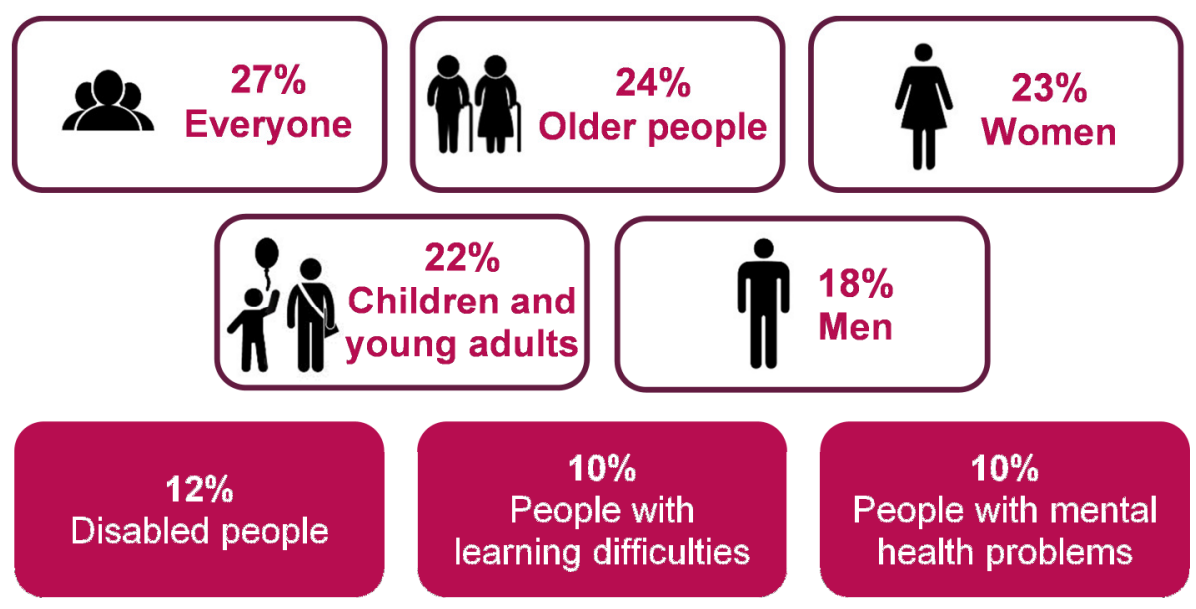


It is estimated that the VCSE sector in Salford made:

\section{4 million interventions}

\section{with clients, users or beneficiaries in the past year}

The VCSE sector works at a

Particular Salford neighbourhoods and range of different geographical levels, both across and beyond Salford. The local authority area, and specific communities and neighbourhoods within it, are the main focus for a majority of organisations. communities

Across the whole

Salford Local

Authority area
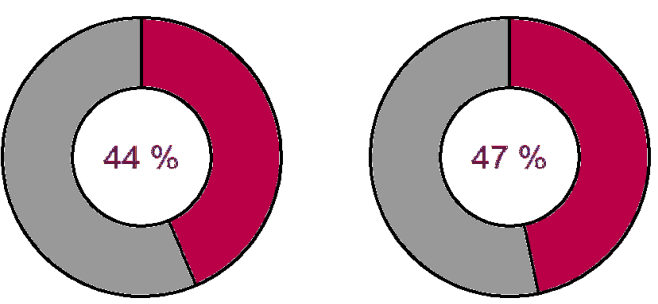

\section{Q3. What does the VCSE sector in Salford do?}

The VCSE sector in Salford works across a diverse range of thematic service areas. However, the proportion of responding organisations working in each area varies. This most likely reflects local needs and funding opportunities. The areas with the greatest proportion of organisations working in them are:
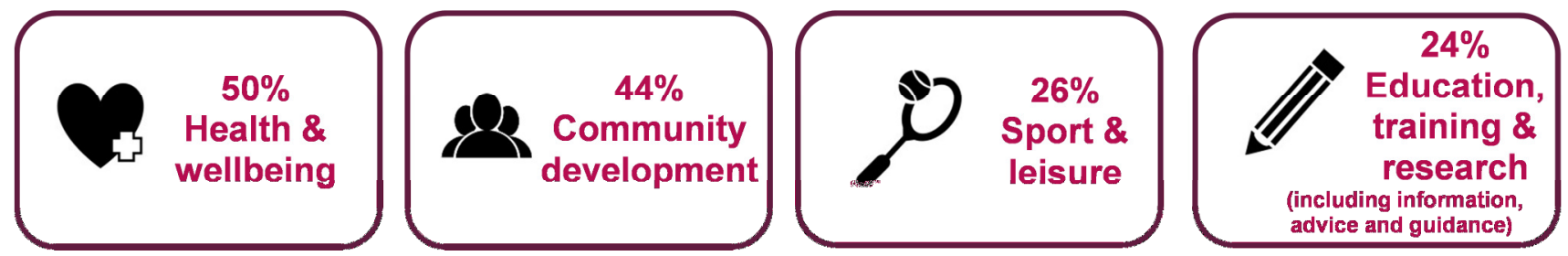

MAIN AREAS IN 2012/13:

HEALTH AND WELLBEING - 47\%

EDUCATION, TRAINING \& RESEARCH - 34\%

COMMUNITY DEVELOPMENT - 41\%

SPORT \& LEISURE- $26 \%$

\section{Q4. What is the income of the VCSE sector in Salford?}

Total income in $2014 / 15$ is estimated to be $£ 165 \mathrm{~m}$, an increase of three per cent compared to 2013/14.

$\%$ change

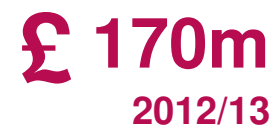

Micro and small organisations account for almost nine out of ten organisations in the VCSE sector in Salford but less than ten per cent of total income.

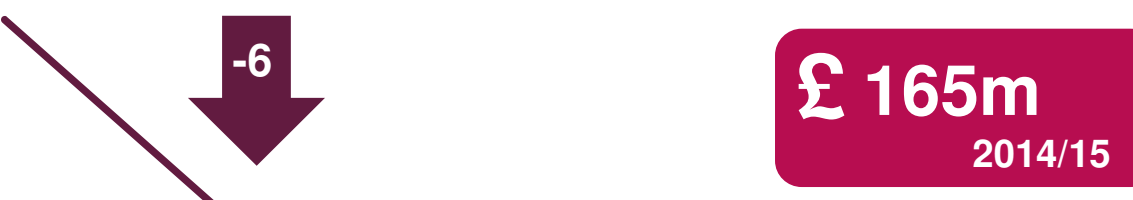

reductions in total income between 2012/13 and 2014/15.

By contrast medium and large organisations saw a reduction in total income between 2012/13 and 2013/14 but then an increase between 2013/14 and 2014/15. However, income is still below 2012/13 levels. 


\section{Q5. Where does the VCSE sector in Salford receive its funding from?}

\section{$79 \%$ have at least one source of non-public sector funds \\ $90 \%$ IN 2012/13}

INCLUDING:

Fundraising (received by 40 per cent of respondents)

Membership fees and subscriptions (32 per cent)

Grants from charitable trusts and foundations (32 per cent)

MAIN SOURCES OF FUNDING IN 2012/13:

FUNDRAISING - 45\%

GRANTS FROM CHARITABLE TRUSTS

\& FOUNDATIONS - $39 \%$

MEMBERSHIPS FEES \& SUBSCRIPTIONS- $31 \%$

\section{$75 \%$ have at least one source of public sector funds \\ $62 \%$ IN 2012/13}

\section{INCLUDING:}

Grant funding administered by Salford CVS on behalf of a public sector body (received by 36 per cent of respondents)

\section{Salford City Council (33 per cent)}

NHS Salford CCG (20 per cent)

MAIN SOURCES OF FUNDING IN 2012/13:

SALFORD CITY COUNCIL - 53\%

NHS SALFORD - $18 \%$

\section{Q6. How sustainable is the VCSE sector in Salford?}

\section{The survey highlights some areas for concern:}

- 56 per cent of respondents reported increasing their expenditure but only 48 per cent had experienced an increase in income

- 24 per cent of respondents reported a decrease in income but only 14 per cent reduced their expenditure

- 25 per cent reported a reduction in their financial reserves compared to 27 per cent reporting an increase.

21 per cent of respondents provided an expenditure figure for 2014/15 that was greater than their income (lower however than the 2012/13 figure of 34 per cent). This means that there were a sizeable number of organisations that spent more money than they received in the past 12 months.

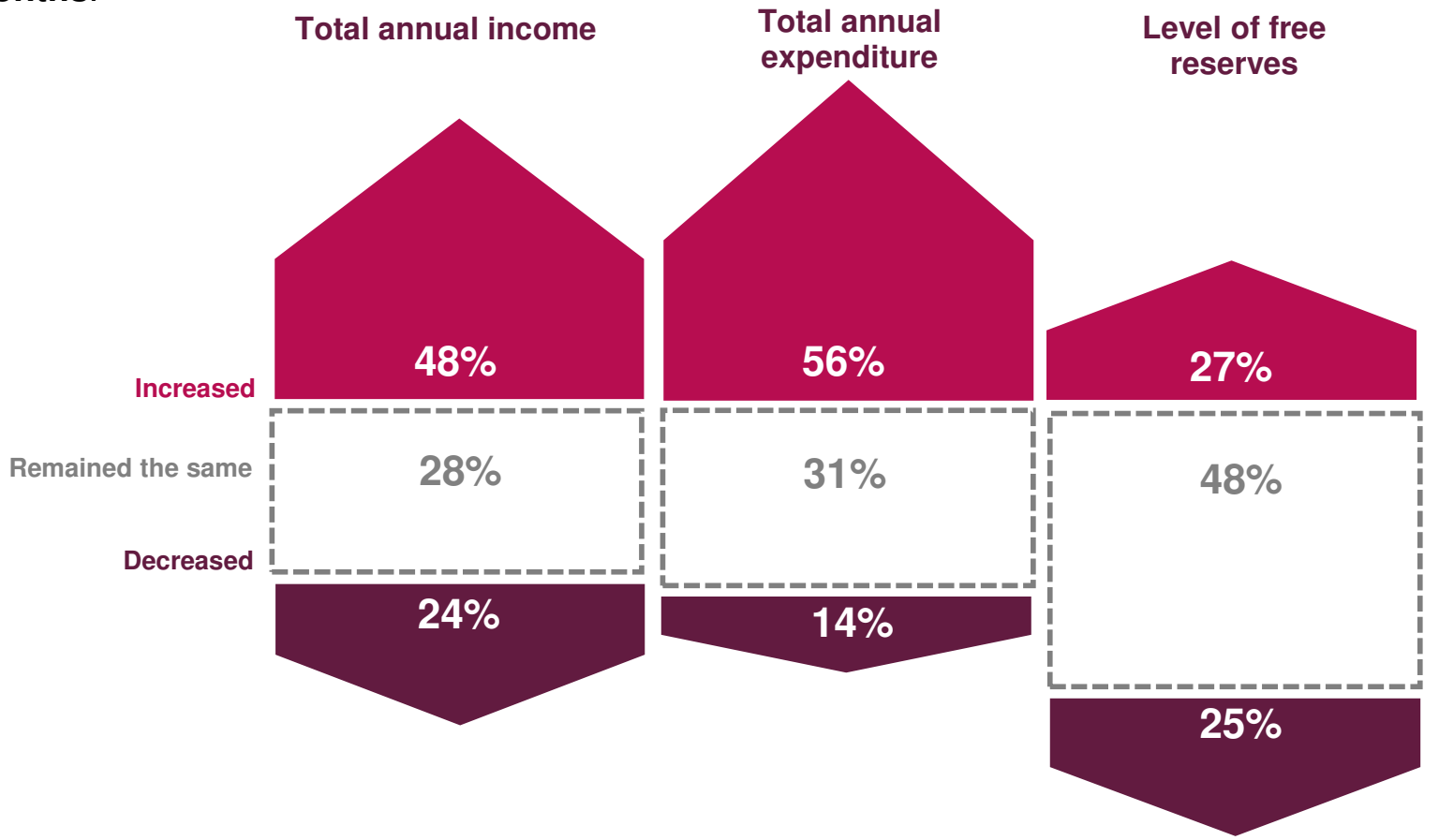


The precarious financial situation of some organisations is further emphasised by the state of their reserves. The figures below suggest that a number of organisations that are dependent on external funding could be particularly vulnerable should their funds be severely reduced or withdrawn.

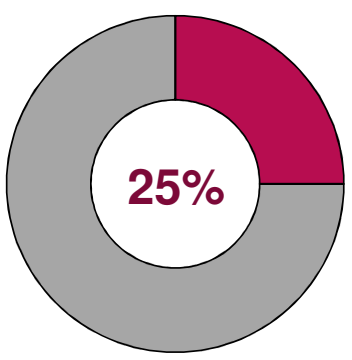

Proportion of organisations with reserves less than one month's expenditure

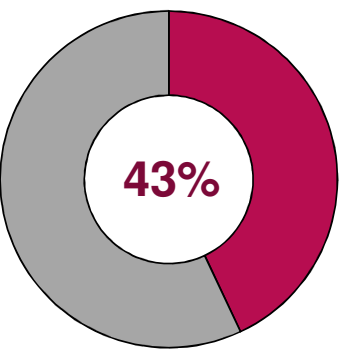

Proportion of organisations with reserves less than

25 per cent of annual expenditure

\section{Q7. Who works and volunteers in the VCSE sector?}

Based on responses to the survey across Greater Manchester on the numbers of volunteers and committee/board members and the hours which they contribute, and drawing on the assumptions used to estimate the total number of organisations in Salford, the following are estimated:

\section{6,800 volunteers $\&$ committeel board members $(36,500$ volunteers and $10,400 \mathrm{committee/board} \mathrm{members)}$}

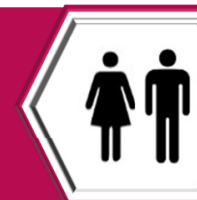

who donated

115,400 hours

of their time per week

$£ 104.4$ million per annum

= estimated economic contribution

of volunteers

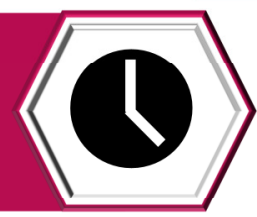

These estimates indicate the ongoing importance of the sector in the City. The VCSE sector is also a significant employer. There are an estimated:

\section{3,500 FTE paid staff $(5,300$ employees in total) employed in the VCSE sector in Salford}

\section{111.2 million per annum contributed to the economy by paid employees of Salford VCSE sector organisations}




\section{Q8. How good are relationships with public sector bodies?}

Survey respondents had dealings with a range of local public sector bodies. The three most prominent were:

\section{$78 \%$ had some dealings with Salford City Council \\ $58 \%$ had some dealings with Salford schools \\ $50 \%$ had some dealings with Salford University}

This highlights the continuing importance of the City Council, Salford schools, and the University to the VCSE sector's work. The relationship between the sector and its local public sector partners is therefore crucial to its ability to operate effectively. To this end survey respondents were asked about the quality and effectiveness of their relationships with key public sector bodies:

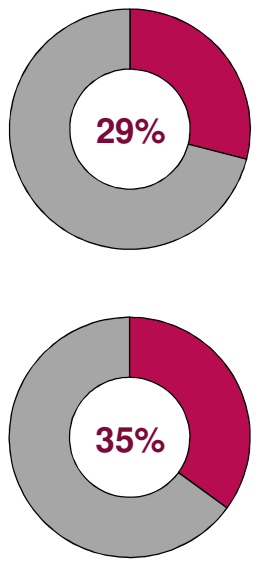

... of VCSE organisations were satisfied with their ability to influence Salford City Council, up from just 16 per cent in 2012/13; more respondents (41 per cent) were satisfied with their ability to influence their most frequent other public sector contact, however this is likely to be a chosen relationship, fulfilling a different role to any relationship with the Council

... of VCSE organisations felt Salford City Council is a positive influence on their success, identical to the proportion in 2012/13. More respondents (57 per cent) felt their most frequent other public sector contact was a positive influence on their success, again perhaps reflecting the chosen nature of this relationship

Respondents were also asked to consider the extent to which their organisation has direct dealings with any emerging Greater Manchester structures (e.g. Greater Manchester Combined Authority, The Office of the Police and Crime Commissioner, The Health and Social Care Devolution Team etc.).

$35 \%$ had some dealings with any emerging Greater Manchester structures

\section{Q9. How well does the VCSE sector work with private businesses?}

Working with private businesses still appears to be new territory for some VCSE organisations, however perceptions of private businesses appears to have improved over time.

\section{$55 \%$ had some dealings with local private businesses}

... 33 per cent of respondents felt that the private business community in Salford was a positive influence on their organisation's success - this is an increase of 12 percentage points since $2012 / 13$

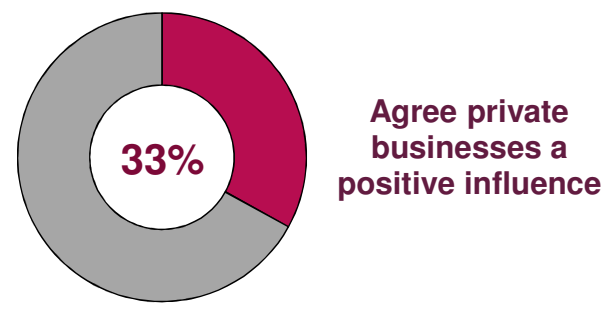

\section{$2 \%$ are members of a private sector-led Consortium}




\section{Q10. How well does the VCSE sector work together?}

The VCSE sector in Salford continues to be well connected internally although most contact appears to be informal.

91\% had some contact with other VCSE organisations in Salford (40 per cent with VCSE organisations in Greater Manchester). $67 \%$ had a 'great' or 'fair amount' of contact.
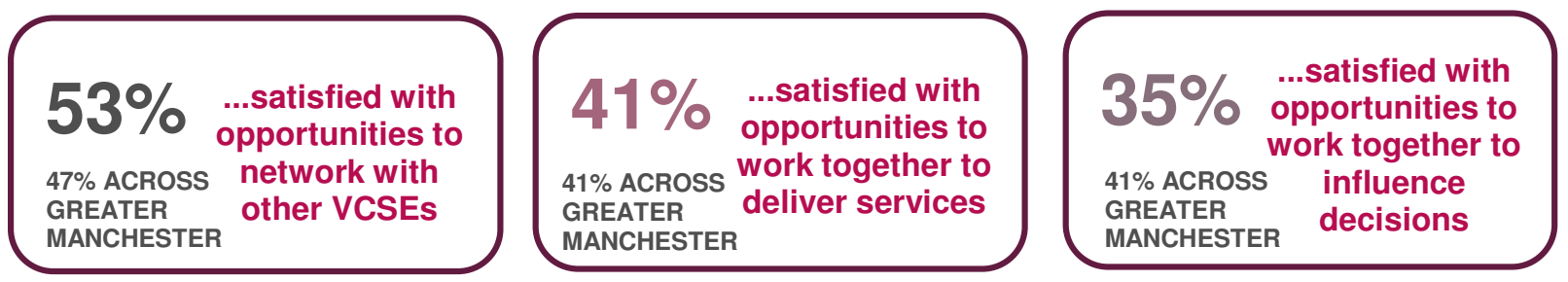

$28 \%$ are members of a formal VCSE consortium

\section{Q11. What are the key issues facing the VCSE sector in the future?}

Respondents were asked about the strategies they are actively pursuing or planning to pursue. Over half of respondents were already doing or planning to do the following:

\section{$59 \%$ work more closely with another voluntary/not-for-profit organisation $57 \%$ increase earned income $52 \%$ change the way they run services or activities}

Respondents were also asked to consider the factors they anticipated assisting or constraining their organisation over the next 12 months:

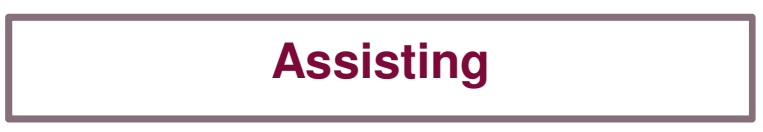

TOP FACTORS:

Engagement with other VCSE organisations (53 per cent assisting or greatly assisting)

Ability to retain staff/staff turnover (53 per cent)

Ability to retain volunteers/volunteer turnover (51 per cent)

\section{Constraining}

\section{TOP FACTORS:}

The local economy (40 per cent constraining or seriously constraining)

Ability to secure contracts from public sector bodies (32 per cent) 


\section{Introduction}

This report provides the main findings of research aimed at improving the understanding of the social and economic impact of the voluntary, community and social enterprise (VCSE) sector in Salford. The research was commissioned by Salford CVS as part of $10 \mathrm{GM}^{4}$ with GMCVO and undertaken by the Centre for Regional Economic and Social Research (CRESR) at Sheffield Hallam University.

The key objective of the research was to provide a comprehensive overview of the sector in Salford at the start of 2017.

The research involved a web-based survey of organisations supporting the people and communities of Salford and focus groups with VCSE organisations. The research took place between September 2016 and January 2017.

Appendix 1 provides further detail on the research methodology.

${ }^{4} 10 \mathrm{GM}$ is a joint venture by the Greater Manchester Voluntary Sector Infrastructure Organisations including Salford CVS (lead partner on this research), Action Together in Oldham and Tameside, Bolton CVS, CVS Rochdale, Macc and Wigan and Leigh CVS. 


\section{Context for the Research}

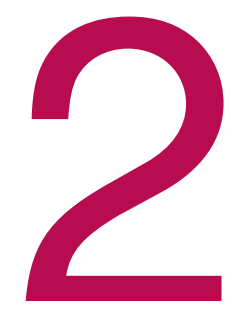

This research comes during both a period of slow economic recovery following the recent long-term economic downturn and a rapidly changing political backdrop as the UK prepares to exit the European Union and the devolution agenda gains pace.

NCVO report that between 2012/13 and 2013/14 the income and spending of the voluntary and community sector in the UK increased, the first notable net growth since the peaks of 2007/08 and 2009/10 respectively. ${ }^{5}$ Total income has increased by just over £2.4bn to £43.8bn and now exceeds the 'peak income' seen in 2007/08 (£43.2bn). NCVO also report that following a decrease in income from government after 2009/10, income from government increased between 2012/13 and 2013/14 by around £0.5bn, although this remains below 2009/10 levels. The majority of this increase was in the largest charities which means the impact might not be felt as keenly at a local level. Income from individuals has also increased by just over £1bn between 2012/13 and 2013/14 and is now at its highest ever level.

However, while the figures above provide reasons to be optimistic there is still need for caution. With the election of the Conservative Government in May 2015, austerity measures are set to continue for the foreseeable future and VCSE organisations are likely to feel the impact of these measures. This includes the Government's commitment to a continuing programme of welfare reform which is likely to result in increasing demand for some services as benefits are restricted or withdrawn. The total anticipated reduction by $2020 / 21$, from both pre and post-2015 welfare reforms in Salford, is predicted to be $£ 135 \mathrm{~m}$ per year or equivalent to $£ 850$ per working age adult per year. ${ }^{67}$ These reforms are likely to continue to put pressure on VCSE organisations both in terms of their financial health and the need to meet greater levels of need from existing and new beneficiaries.

Locally, the reductions in public expenditure have been felt acutely in Salford. As part of the Coalition Government's plan to reduce the deficit, it reduced funding for local government in England. Across Greater Manchester local authorities have experienced, and are continuing to experience, a decline in Government funding. Salford City Council has made $£ 186$ million of savings since 2010 , due to an overall 47.4 per cent reduction in government funding ${ }^{8}$.

\footnotetext{
${ }^{5}$ UK Civil Society Almanac (2016) NCVO.

${ }^{6}$ Beatty and Fothergill (2016) The Uneven Impact of Welfare Reform: The financial losses to places and people.

${ }^{7}$ Note: These figures are based on HMRC Budgets and Autumn Statements from between 2010 and 2015. In the 2016 Autumn statement the Pay-to-stay measure was scrapped and so this has been taken account of in the figures. The estimate of cuts due to the LHA Cap in social housing was increased by a further $£ 160 \mathrm{~m} \mathrm{p.a.} \mathrm{which}$ is not taken account of in the figures. The Universal Credit Taper was also increased by $2 p$ in the pound, an increase in funding of $£ 570 \mathrm{~m}$ p.a., which is not included in the figures presented here.

${ }^{8}$ Source: Salford City Council - website
} 
At a local level there have also been significant changes which have impacted on the environment in which the sector operates. May 2012 saw the election of Salford's first directly elected City Mayor and following this the cabinet system of local Government was replaced by a Mayoral Team.

In 2016 Paul Dennett was elected as the second Mayor of Salford. Salford also has a City Partnership which comprises numerous partners from across different sectors, with thematic groups having VCSE sector reps on them. There is a small City Leaders Group, convened by the City Mayor of which Salford CVS' CEO is a member.

December 2012 saw the establishment of a Third Sector Consortium, supported by Salford CVS. This now comprises of 85 VCSE organisations. Salford CVS employs its staff and provides financial and management services to the consortium, which has annual contracts approaching $£ 500,000$. Salford is also a Social Enterprise City and the first place in the North West to be an accredited place by Social Enterprise UK. Salford CVS has successfully worked in partnership with NHS Salford CCG since its creation and established the CCG funded Third Sector Fund, which provides grants totalling $£ 1 \mathrm{~m}$ to local VCSE organisations and primary schools.

Salford has a Poverty Truth Commission, comprising 'lived experience' and 'public life' commissioners, including Salford CVS, CAB, Credit Union, RSLS, FE college, CCG, Council, business. In February 2017 Salford launched a new strategy 'Tackling Poverty: No One Left Behind' which includes commitments around social housing, investing in the safety net (welfare rights, debt advice, etc.) and the local credit union, challenging welfare reform, etc.

There are 12,667 Salford children estimated to be living in poverty in 2016 (23 per cent of children). According to the national Index of Multiple Deprivation (IMD) Salford is ranked as the 22nd most deprived local authority area in England (out of 326). Levels of poverty and deprivation across Salford also vary significantly, with the most deprived wards being Langworthy, Broughton, and Little Hulton, and the least deprived being Worsley, Boothstown and Ellenbrook, and Claremont. The link between poverty and a higher risk of illness and premature death is well established. In Salford, people living in some of our poorest areas are living up to 14 years less than those in more affluent ones. 20,200 people aged 16 and over in Salford (10 per cent of population) belong to an ethnic minority and 16,085 people do not speak English as their main language (with over 70 languages being spoken in total). This continues to be a significant barrier in reducing poverty for this vulnerable group.

Homelessness in Salford is also increasing. Since 2013, Salford has seen a 72 per cent increase in the number of people presenting as homeless (from 741 to 1273), and a 73 per cent increase in the number of homeless acceptances e.g. those with a priority need (from 203 to 352 ).

Despite this, Salford continues to receive the lowest Homeless Prevention Grant in the whole of Greater Manchester. Research undertaken by Salford's Financial Inclusion Practitioners Group and the University of Salford also shows that rent arrears across all housing providers are on the increase, with the implementation of Universal Credit seen as a key driver with payments now being monthly in arrears, and housing benefit going directly to the claimant and not the landlord.

Against this background this research provides in depth data about the 'state of the VCSE sector' in Salford at the start of 2017. The research provides a comprehensive overview of the sector in Salford for partners to draw upon and help harness the potential of the sector. 


\section{What the Voluntary Community and Social Enterprise Sector in Salford does}

This chapter develops a picture of the core features of the voluntary, community and social enterprise (VCSE) sector in Salford. It focuses on a series of general questions in which respondents were asked about their group or organisation: what it is and what it does.

\subsection{How many VCSE organisations are there in Salford?}

Estimating the number of organisations represents a major challenge. This is because a large proportion of organisations are small, local and not formally constituted as charities, limited companies or other recognised forms which require registration (e.g. industrial and provident societies). As a result they do not appear on formal central records such as those held by the Charity Commission or Companies House so are considered 'below the radar' (BTR). Any estimate of the total number of organisations in an area therefore requires information on the numbers of registered and unregistered (i.e. BTR) organisations.

In estimating the total number of organisations in Salford we drew on information from the following sources:

- The Register of Charities in England and Wales, which indicated 479 registered charities with postcodes in Salford.

- The ratio of charities to non-charities provided in the 'National Survey of Charities and Social Enterprises' (NSCSE), undertaken by Ipsos MORI for the Cabinet Office in 2010. This was used to gross the estimate upwards to a total of 614 registered organisations, to take account of non-charitable social enterprises.

- Research by NCVO and the University of Southampton ${ }^{9}$ which found that on average there are 3.66 BTR organisations per 1,000 population. If this figure is applied to Salford ${ }^{10}$, it can be estimated that there are 899 BTR organisations in the City. ${ }^{11}$

\footnotetext{
${ }^{9}$ Mohan, J et al. (2010). Beyond 'flat-earth' maps of the third sector: enhancing our understanding of the contribution of 'below-the-radar' organisations. Northern Rock Foundation Briefing Paper

${ }_{11}^{10}$ Based on Office for National Statistics 2015 population estimates

${ }^{11}$ It is important to note that the BTR figure is an estimate based on an average across 46 local authorities. The BTR research found significant variability, with some local authorities reaching over seven BTR organisations per 1,000 population, and in one case exceeding 10 .
} 
Summing the estimated numbers for both registered and BTR organisations produces an estimated figure of:

\section{1,513 organisations in total operating in the VCSE sector in Salford.}

This is higher than the estimate produced for the 2013 report $(1,364)$. Whilst this may reflect a genuine increase in the number of voluntary organisations between the two surveys this could also in part be due to unavoidable differences in the estimation methodology.

For the 2013 report, the sampling frame for the NSCSE was used to provide the estimates for the number of formally registered organisations. Unfortunately this survey was subsequently cancelled and has not been repeated. As such, only the ratio of charities to non-charities was taken from the sampling frame for the NSCSE for the latest study. This ratio was used to gross up the number of charities from the charity register.

\subsection{What size are organisations in Salford?}

The size of organisations is traditionally measured using their annual income ${ }^{12}$. When the distribution of organisations across Salford was explored by size category based on income for 2014/15, it showed that the majority of organisations were either micro or small. But the survey was under-representative of BTR organisations (only 42 per cent of survey respondents were identified as BTR), so this did not present an accurate picture of the actual distribution. The figures were therefore adjusted based on the assumption that the estimated 649 organisations not included in the survey sample were BTR and micro in size ${ }^{13}$.

Exploring the distribution by size category based on income for 2014/15 across Greater Manchester also showed some inconsistency with the distribution found in the earlier 2013 study. Therefore, in order to provide the most robust estimate of the distribution of organisations in the VCSE sector by size, data from both the 2012/13 and 2016/17 waves of the survey have been used to calculate the proportion of organisations estimated to be in each size category.

The outcome of this process is shown in figure 3.1 , which demonstrates that an estimated: 69 per cent of the VCSE sector (1,038 organisations) are micro in size, 18 per cent are small (271 organisations), 10 per cent are medium (158 organisations), and three per cent are large (46 organisations).

Introducing the BTR figure produces a much higher estimate for the number and proportion of micro organisations and emphasises the finding that a large proportion of organisations in the VCSE sector in Salford are very small (87 per cent micro or small). This is similar to the national picture: $\mathrm{NCVO}^{14}$ estimate that 83 per cent of the VCSE sector is made up of micro or small organisations, 14 per cent are medium, and three per cent are large. Results are also consistent with the pattern across Greater Manchester as whole, where 90 per cent of organisations are micro or small, eight per cent are medium and two per cent are large.

\footnotetext{
${ }^{12}$ In exploring organisation size we used the categories developed by NCVO for use in their Almanac series (see e.g. Clark, J et al., 2010)

${ }^{13}$ The basis for these assumptions is discussed in more detail in the methodological annex

${ }^{14}$ UK Civil Society Almanac (2016) NCVO.
} 


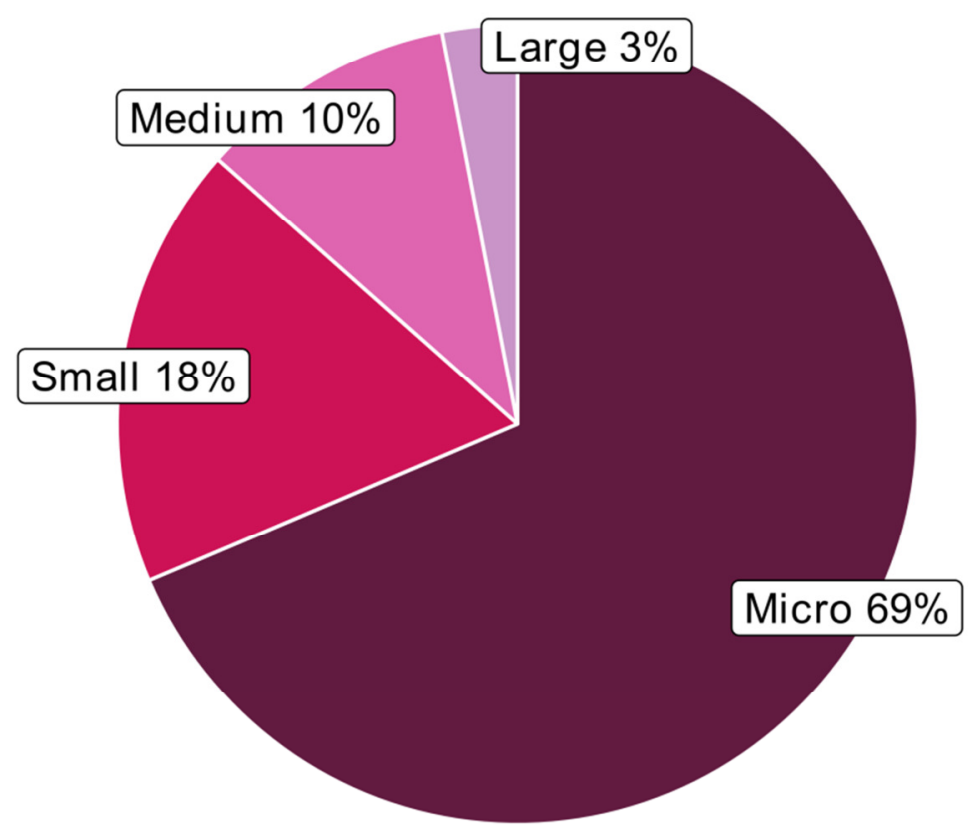

Source: Salford State of the VCSE sector survey 2016/17

Base: 115

\subsection{What types of organisations operate in the VCSE sector in Salford?}

Respondents to the questionnaire were asked to identify which category from a list of 'organisation types' best described their organisation. The results indicate that many organisations in the VCSE sector are likely to have a local focus. Figure 3.2 shows that the largest proportion, 21 per cent, identified their organisation as being a local voluntary organisation. There were three other types of organisation which over one in ten respondents identified themselves as: community or neighbourhood group (16 per cent); social enterprise (14 per cent); and sport, leisure or social group (11 per cent). National organisations were less common: just over one in ten organisations stated they were either a national voluntary organisation (three per cent), a branch of a national voluntary organisation (five per cent) or an affiliated member of a national voluntary organisation (five per cent).

This breakdown of organisations by type followed a similar pattern to that in the 2012/13 and 2010 surveys, where the two largest categories were: local voluntary organisation (31 per cent and 27 per cent) and community or neighbourhood group (13 per cent and 14 per cent respectively). National organisations were also less common in 2012/13 and 2010.

The proportion identifying as a social enterprise has increased from just six per cent in 2012/13 to 14 per cent in the latest survey. This is also higher than the Greater Manchester figure of eight per cent. This could potentially be a reflection of Salford now being a Social Enterprise City and the first place in the North West to be an accredited place by Social Enterprise UK. 
The analysis across Greater Manchester found a similar picture with local voluntary organisations (22 per cent) and community or neighbourhood groups (15 per cent) accounting for 38 per cent of respondents. Only 13 per cent of respondents indicated they were either a national voluntary organisation (two per cent), a branch of a national voluntary organisation (six per cent) or an affiliated member of a national voluntary organisation (four per cent).

Figure 3.2: Type of organisations

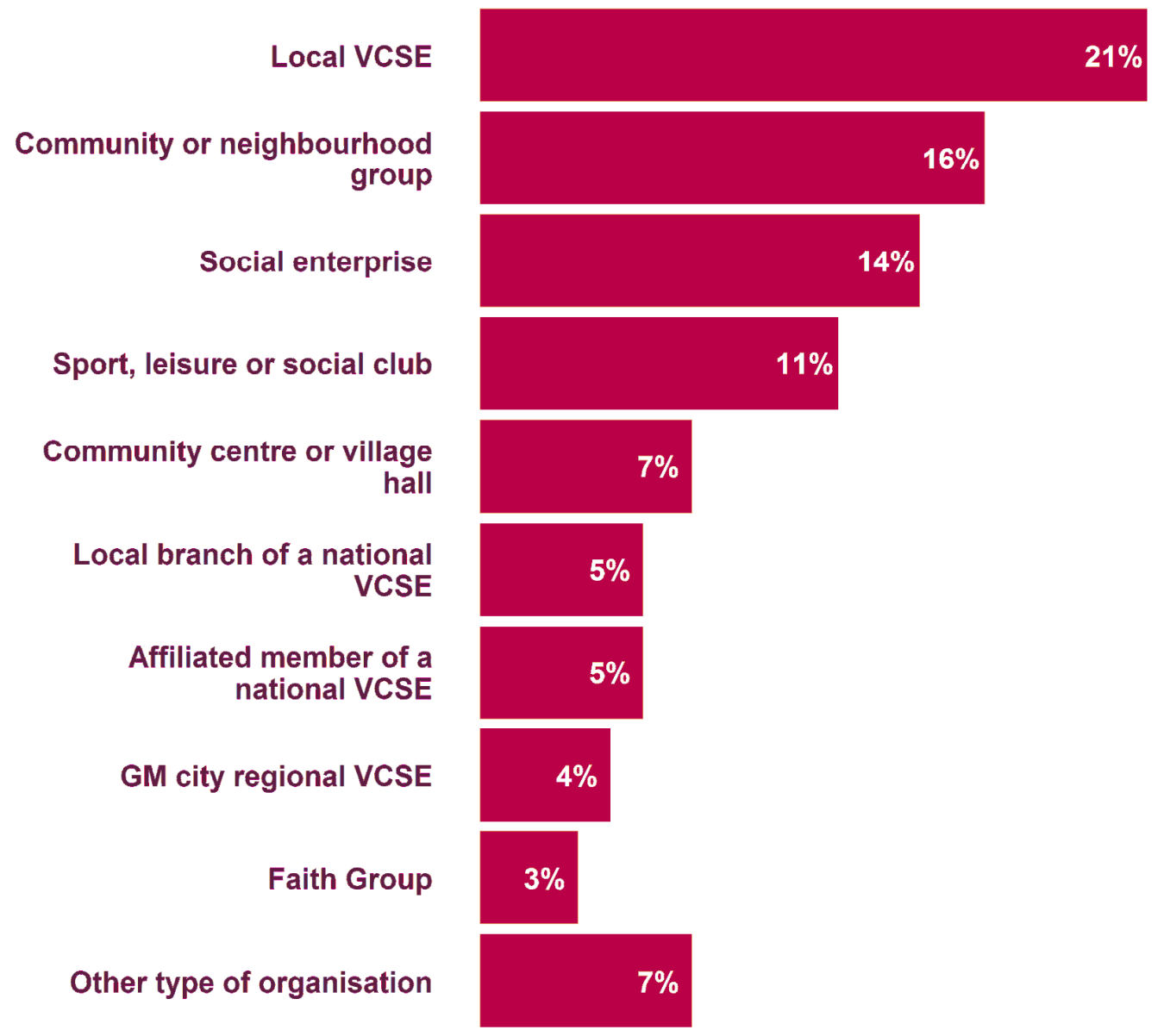

Source: Salford State of the VCSE sector survey 2016/17

Base: 197

\subsection{How long have organisations in the VCSE sector been operating?}

The questionnaire asked respondents to indicate when their organisation was formed. Assessment of organisations by the year in which they were formed provides an indication of how established the VCSE sector is in Salford.

The responses received build a picture of a VCSE sector that has a fairly well established core. However, the VCSE sector in Salford has also seen the formation of many new organisations since 2001. Figure 3.3 shows that 62 per cent of organisations responding to the survey had been formed since 2001, including 49 per cent in the past 10 years (i.e. since 2006). Furthermore, an additional 13 per cent were formed between 1991 and 2000; this means three-quarters (75 per cent) of organisations were formed in the last 25 years. At the other end of the spectrum 11 per cent of organisations had been formed before 1971, including three per cent formed in 1910 or before. 
In the 2012/13 survey, 64 per cent of organisations were formed since 1991 (55 per cent in the 2010 survey), including 37 per cent, which had been formed in the past 10 years (36 per cent in 2010). Four per cent of organisations in 2012/13 had been formed in 1910 or before, compared with three per cent in the 2016/17 survey.

The pattern for organisations responding to all of the Greater Manchester surveys was broadly similar. 43 per cent of respondents had been formed in the past 10 years, although a lower figure, 29 per cent of organisations had been formed since 2011. Six per cent of Greater Manchester organisations had been formed before 1911.

Figure 3.3: Year in which organisations were formed

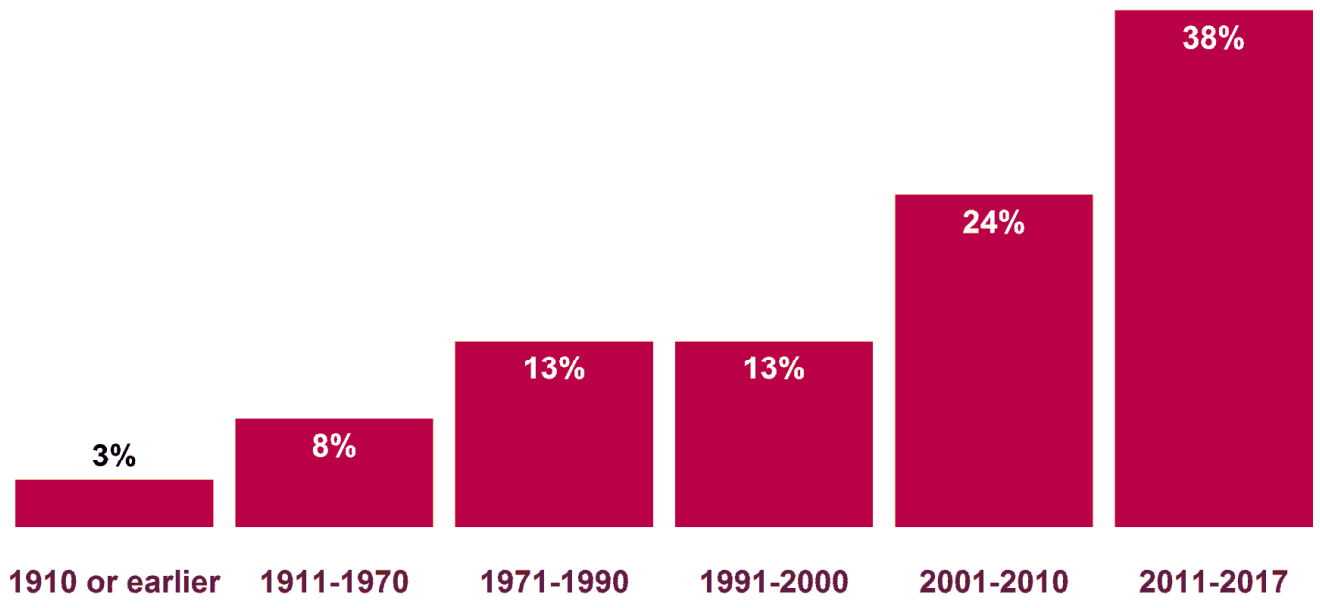

Source: Salford State of the VCSE sector survey 2016/17

Base: 178

It is important to conclude this section by drawing an important qualification. Although the results suggest that it is likely that the VCSE sector in Salford has experienced growth in the number of organisations established in the last 20 years or so, it may not be as dramatic as the figures suggest. By definition, the survey is of organisations still operating in Salford in 2016/17, not those which have closed down or ceased operations. Of the organisations which have survived through to 2016/17, the results suggest that a high proportion were established in the last 20 years. But some of the organisations established before, and since, may have subsequently closed down. Because we do not know the rate of closure over time we cannot be certain that the aggregate number of organisations being established or surviving is increasing.

\subsection{What does the VCSE sector in Salford do?}

To elicit a picture of what the VCSE sector in Salford does, the survey asked respondents to identify up to three main areas in which their organisation operates. Figure 3.4 presents the top ten main areas selected and confirms the message that the VCSE sector in Salford works in a diverse range of thematic service areas. However, the proportion of responding organisations working in each area varies. This is most likely dependent on need and funding opportunities. 
Figure 3.4 shows:

- 50 per cent of organisations worked in the area of health and wellbeing; in 2012/13 and 2010 this area was also the most common main area (47 per cent and 42 per cent of organisations respectively)

- 44 per cent worked in community development, similar to the $2012 / 13$ survey (41 per cent, which was some 11 percentage points higher than the 2010 survey)

- $\quad$ over one fifth of organisations worked in each of the following themes:

- $\quad$ sport and leisure (26 per cent; identical to the $2012 / 13$ proportion)

- education, training and research (24 per cent compared to 34 per cent in 2012/13).

Across Greater Manchester as a whole the same four areas of work were reported as being the most common to work within:

- $\quad$ health and wellbeing (46 per cent)

- community development (39 per cent)

- $\quad$ education, training and research (26 per cent)

- $\quad$ sport and leisure (25 per cent).

Figure 3.4: Top 10 main areas in which organisations work

Health and well-being
Community development

Sport and leisure

Education, training and research (including information, advice and guidance)

Economic well-being

Arts, heritage and culture

Capacity building and other support for voluntary or community organisations

Environment and sustainability

Social care

Other charitable, social or community purpose

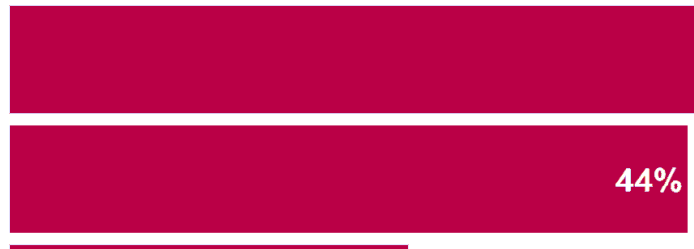

$26 \%$

$24 \%$

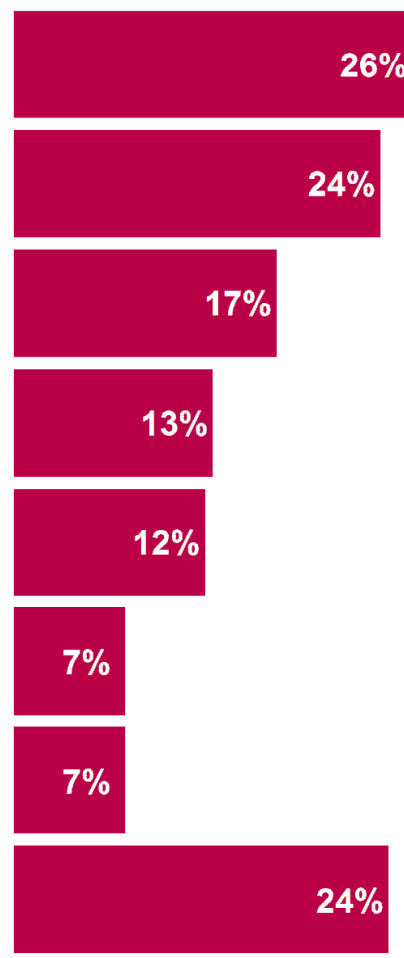

Source: Salford State of the VCSE sector survey 2016/17

Base: 195 
In the latest survey respondents who indicated they worked in the area of health and wellbeing were asked to specify the specific areas in which they operate. The majority (84 per cent) stated they worked in health and wellbeing in general. Over half (54 per cent) indicated they worked in mental health. In addition over one third said they worked in healthy living (food \& lifestyle, sexual health) (38 per cent) or disability or sensory impairment (34 per cent). Responses were very similar for Greater Manchester overall.

In a similar vein, respondents who identified education, training and research as a main area of work were asked to specify the areas they worked within this theme. Of the 43 respondents who answered this question 29 (67 per cent) stated they worked in education, while 28 (65 per cent) said they worked in information, advice and guidance. 23 respondents (53 per cent) indicated they worked in the area of employability skills. Responses were again very similar at the Greater Manchester level.

The four per cent of organisations responding to the survey who indicated that equalities and civil rights was a main area of their work were also asked to identify the specific areas within this category in which they operate. The majority (six respondents) stated they worked in the area of disability. All other options received a lower response. The area of disability was also the most common response across Greater Manchester. 


\section{Who the Voluntary Community and Social Enterprise Sector in Salford works with}

This chapter focuses on who the VCSE sector in Salford works with and where.

\subsection{Who are the clients, users or beneficiaries of the VCSE sector in Salford?}

The questionnaire asked responding Salford based organisations to provide the total number of individual clients, users or beneficiaries that their organisation had supported in the last year, both overall (i.e. individuals resident both within Salford and beyond) and just within Salford. Analysis of responses to this question by size and type of organisation revealed that in many cases organisations had provided the number of 'interventions' or 'contacts' that they had had with clients, users or beneficiaries. So for example an individual who visited a community centre once a week would have been counted 52 times within the year. Whilst some organisations will have provided the number of unique clients, users or beneficiaries, so as not to overestimate, in our analysis we have assumed the number provided represents the total number of interventions.

Summing across the 173 organisations that responded gives a total of 914,000 interventions overall. Doing the same for the 174 organisations who provided a figure for Salford gives a total of 576,000 interventions in Salford. The responses received can be extrapolated for the estimated 1,513 organisations thought to be operating in the VCSE sector in Salford to provide an estimate of the total number of interventions by Salford organisations. Working through the calculation it is estimated that Salford organisations had:

\section{4 million interventions with clients, users or beneficiaries in the past year overall}

\section{1 million interventions with clients, users or beneficiaries in the past year in Salford}

The 2012/13 study estimated that Salford organisations made 2.7 million interventions with clients, users or beneficiaries overall.

The questionnaire also asked respondents to identify up to three groups that make up the main clients, users or beneficiaries of their organisation. 
Figure 4.1 shows that, as might be expected, the VCSE sector in Salford serves a diverse and wide population. In many cases, client groups are served by relatively small numbers of organisations: 10 per cent of organisations or fewer serve 16 of the client groups listed.

Figure 4.1 shows that the client groups served by the largest proportions of organisations can be broadly characterised as being demographic: gender - women (23 per cent) and men (18 per cent) - and age - older people (24 per cent) and children and young adults (22 per cent).

General and demographic client groups were also the most common groups identified in both the 2012/13 and 2010 surveys, although the ordering was different. In 2012/13 the most common client groups were women (32 per cent) and men (30 per cent); while in 2010 young people (28 per cent) and children (27 per cent) were the most commonly listed main groups worked with.

Just over one quarter (27 per cent) of organisations identify 'everyone' as their main clients, users or beneficiaries. Individuals with health issues are also served by relatively high proportions of organisations. Disabled people are a main client, user or beneficiary group for 12 per cent of organisations and people with mental health problems make up a main client, user or beneficiary group for 10 per cent of organisations. In the 2012/13 survey these proportions were 18 per cent for disabled people and 16 per cent for people with mental health problems respectively (compared to 15 per cent and 12 per cent in 2010).

Analysis of responses to the Greater Manchester survey found a broadly similar pattern with general and demographic client groups also being the most common beneficiary groups identified:

- $\quad$ everyone: 33 per cent

- $\quad$ children and young adults: 23 per cent

- $\quad$ older people: 17 per cent

- $\quad$ women: 15 per cent

- men: 12 per cent. 
Figure 4.1: Top 10 main client groups of Salford organisations

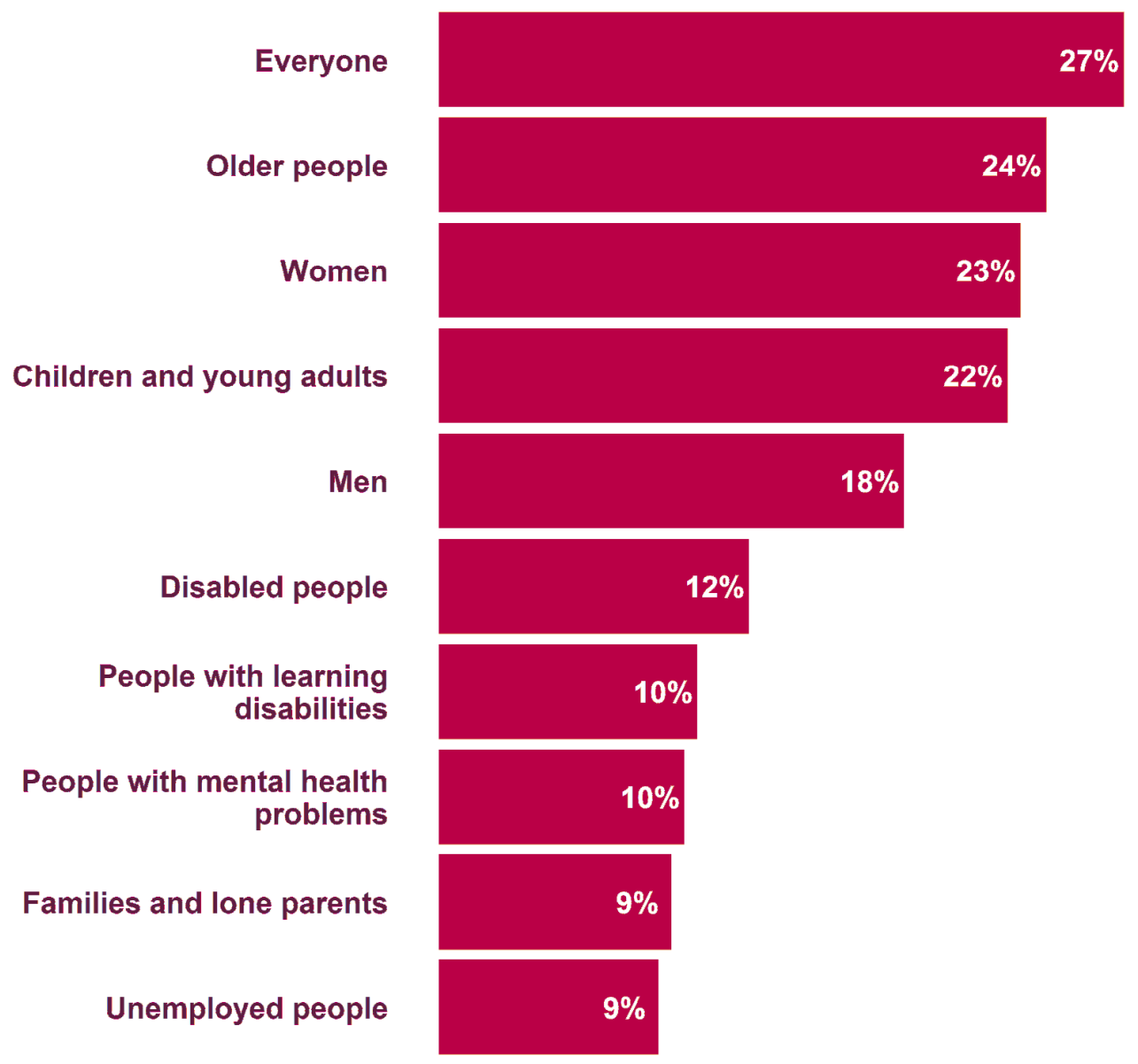

Source: Salford State of the VCSE sector survey 2016/17

Base: 196

Respondents to the 2016/17 survey were asked to identify the ways in which their organisation makes a difference for its service users/client group(s). This question demonstrates the key role that the VCSE sector has in fostering strong and cohesive communities within Salford and highlights the importance of the VCSE sector as an essential part of the social fabric of the City. As figure 4.2 shows, over three-quarters (77 per cent) of organisations felt they are improving people's mental wellbeing while two-thirds (65 per cent) stated they are increasing people's skills. Improving people's mental wellbeing was also the most common response across Greater Manchester. Just over three-fifths also either said they are improving people's physical wellbeing (63 per cent); addressing the needs of disadvantaged members of the community (62 per cent); or encouraging people to get involved in activities and events that are happening in their local area (62 per cent). 
Figure 4.2: Top 10 ways in which organisations make a difference

Improving people's mental wellbeing

Increasing people's skills

Improving people's physical wellbeing

Encouraging people to get involved in activities and events that are happening

Addressing the needs of disadvantaged members of the community

Helping people to feel that they belong to their neighbourhood

Helping people from different backgrounds to get on well together

Encouraging people to be involved in regular volunteering

Helping people feel that Salford is a good place to live

Helping people to feel safe

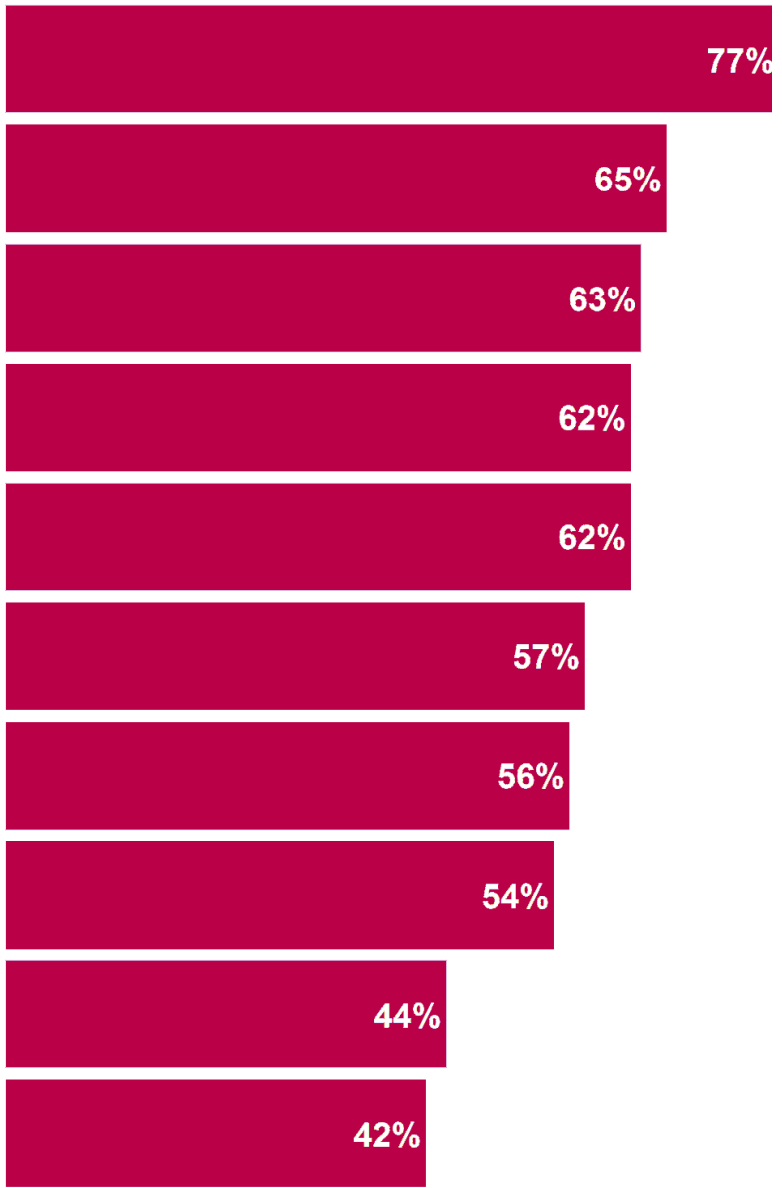

Source: Salford State of the VCSE sector survey 2016/17

Base: 197

\subsection{What geographical levels does the VCSE sector operate at?}

The survey asked respondents to identify the main geographical levels at which they operate - this ranged from the neighbourhood level, to those operating across England, the UK or overseas ${ }^{15}$. In this question respondents were asked to pick out up to three main geographic levels, the results of which are presented in figure 4.3. This shows that the local area is a main focus for a majority of organisations:

- almost half (47 per cent) identified the whole of the Salford local authority area as a main focus of their work; similar to the proportion of organisations in both the 2012/13 and 2010 surveys (both 51 per cent)

- a further 44 per cent identified particular Salford neighbourhoods or communities as a main focus; a similar proportion of organisations (46 per cent) identified this focus in the 2012/13 survey and also the 2010 survey (42 per cent).

${ }^{15}$ This question was asked slightly differently in the latest survey compared to 2012/13. Two additional options ('Across more than one Greater Manchester Local Authority area' and 'Across the whole of Greater Manchester') were included. 
A modest proportion of organisations cited that a main geographic area at which they work was either national (13 per cent) or international (three per cent). In many cases those organisations that work internationally will reflect their main clients, users and beneficiaries.

The picture for Greater Manchester organisations shows that 44 per cent also identified particular neighbourhoods and communities as a main geographic focus, the same proportion as in Salford. The percentage of organisations who said they worked nationally and internationally was very similar within Salford and Greater Manchester.

Figure 4.3: Main geographic focus

\section{Particular Salford neighbourhoods and communities}

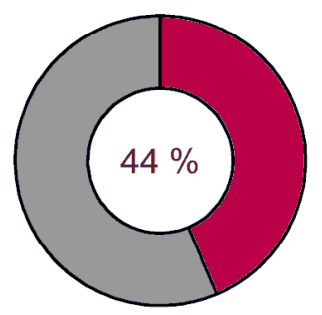

Regionally across the North West

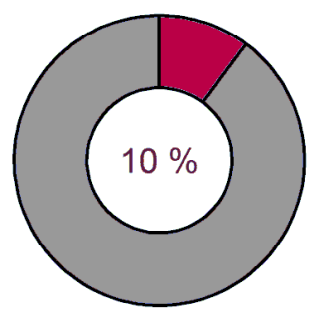

Across the whole Salford Local Authority area

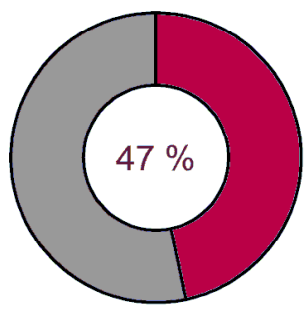

Nationally

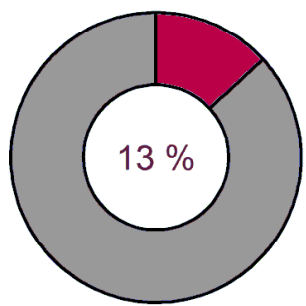

Across more than one Greater Manchester Local Authority area

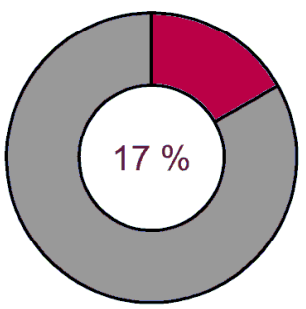

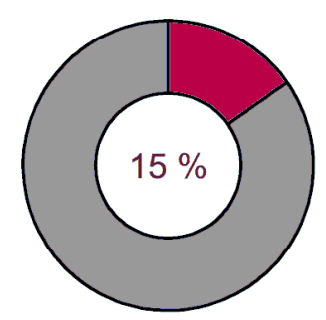

Across the whole of Greater Manchester

Internationally

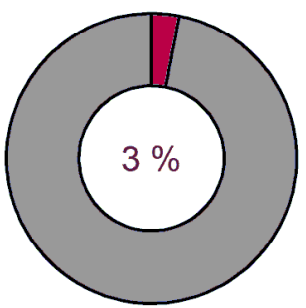

Source: Salford State of the VCSE sector survey 2016/17 Base: 197

Using the responses to this question it is also possible to identify the highest main geographic area that an organisation carries out its activities (see figure 4.4 below). The highest geographic area that could be identified was internationally.

This analysis finds that for over one third (35 per cent) their highest main geographic focus was particular Salford neighbourhoods or communities; similar to the proportion in 2012/13 (33 per cent). This is almost identical to Greater Manchester as a whole where 34 per cent of organisations indicated their highest main geographic focus was particular neighbourhoods and communities. 
Figure 4.4: Highest geographic focus

Particular Salford neighbourhoods and communities

Across the whole Salford Local Authority area

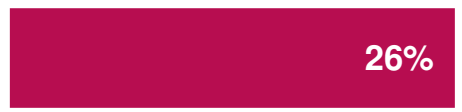

Nationally

$12 \%$

Across more than one Greater Manchester Local Authority area

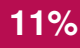

Regionally across the North West

Across the whole of Greater

Manchester

\section{$6 \%$}

Internationally

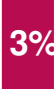

Source: Salford State of the VCSE sector survey 2016/17

Base: 197

Respondents who reported that the whole Salford local authority area or particular Salford neighbourhoods or communities were a main geographic focus of their organisation, were asked to identify in which wards their work focused on. Map 4.1 shows the percentage of all organisations that identified each of Salford's wards as a main focus of their work.

28 per cent of Salford organisations identified Eccles as a main focus of their work. In addition between 20 and 25 per cent of Salford organisations identified Worsley, Swinton South, Kersal, Irlam and Langworthy as a main focus.

The three wards which were a main focus for the lowest proportions of Salford organisations were:

- $\quad$ Boothstown \& Ellenbrook (10 per cent)

- Claremont (11 per cent)

- $\quad$ Irwell Riverside (12 per cent). 
Map 4.1: Percentage of organisations that identify Salford's wards as a main focus of their work

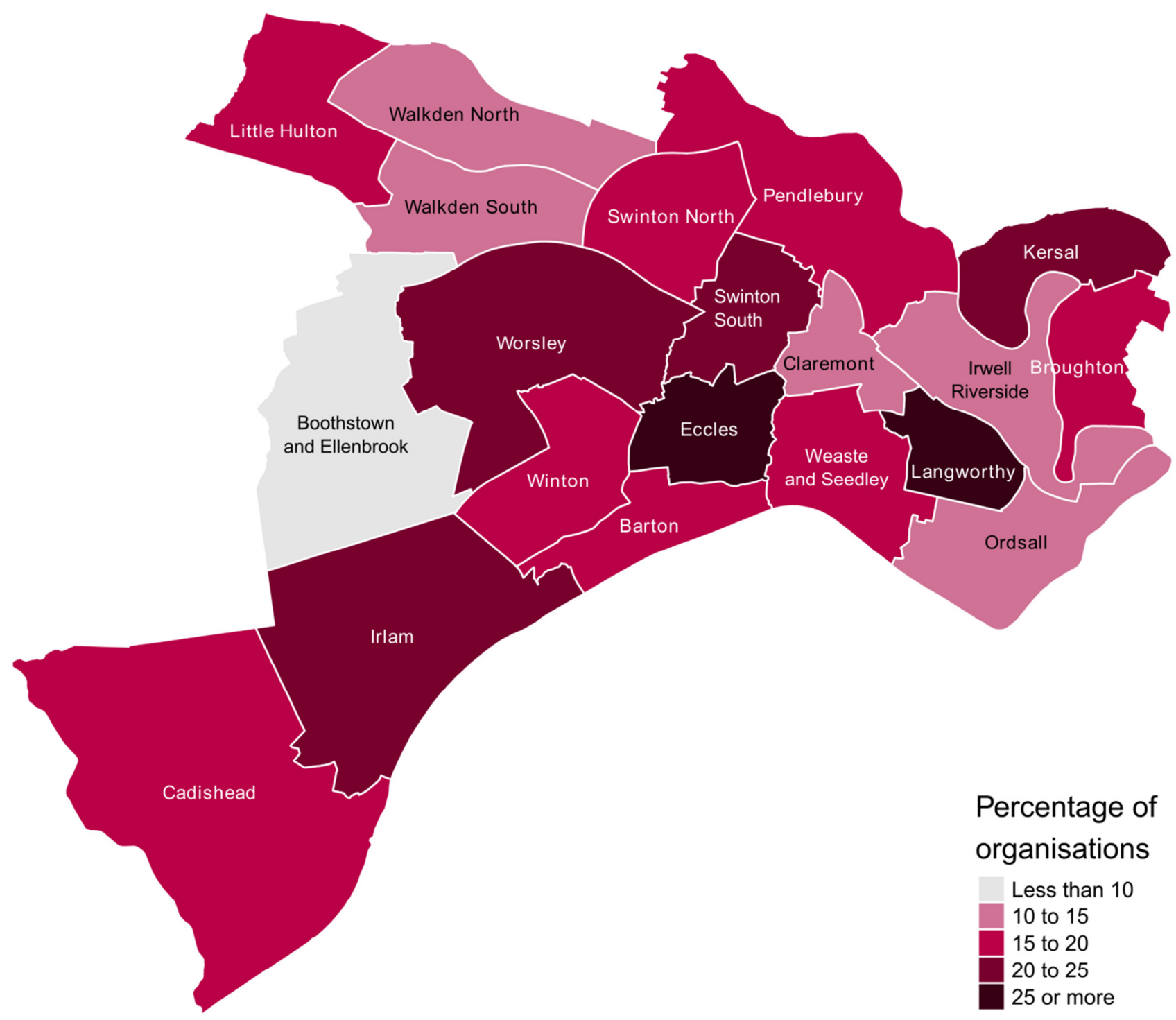

Source: Salford State of the VCSE sector survey 2016/17

Base: 83 


\section{Finances and Income}

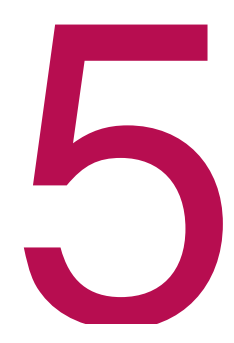

This chapter provides an overview of the finances and income of the VCSE sector in Salford. It includes estimates of the overall income received by the sector between 2012/13 and 2014/15, analysis of the different sources of income received (public sector and non-public sector) and their relative contribution, and an assessment of the financial sustainability of the VCSE sector.

Where possible this chapter compares results from the latest survey and the 2012/13 study. Revisions to the questionnaire and methodology between these studies, however, mean that comparisons are not always possible or appropriate and that caution should be applied when comparing across the two waves (see Appendix 1 for more detail).

\subsection{Income}

Based on the average (mean) income of respondents to the survey across Greater Manchester, and drawing on the assumptions used to estimate the total number of organisations in Salford, the following is estimated ${ }^{16}$ -

\section{$£ 165$ million the total income of the VCSE sector in Salford in 2014/15}

This total income estimate is higher than the figure of $£ 145$ million estimated as being the total income for the sector in 2011/12 from the 2012/13 survey. It also represents an increase of three per cent compared to 2013/14 when the total income of the VCSE sector was an estimated $£ 160$ million. This follows a reduction between $2012 / 13$ and $2013 / 14$ of an estimated six per cent in the total income of the sector, indicating instability in the current financial picture. This data is outlined in more detail in figure 5.1.

\footnotetext{
${ }^{16}$ This figure is based on a weighted average (mean) for each size category for respondents from across Greater Manchester. The methodology is explained in more detail in the methodological appendix.
} 
Figure 5.1: Estimated annual income of the VCSE sector in Salford (2012/132014/15)

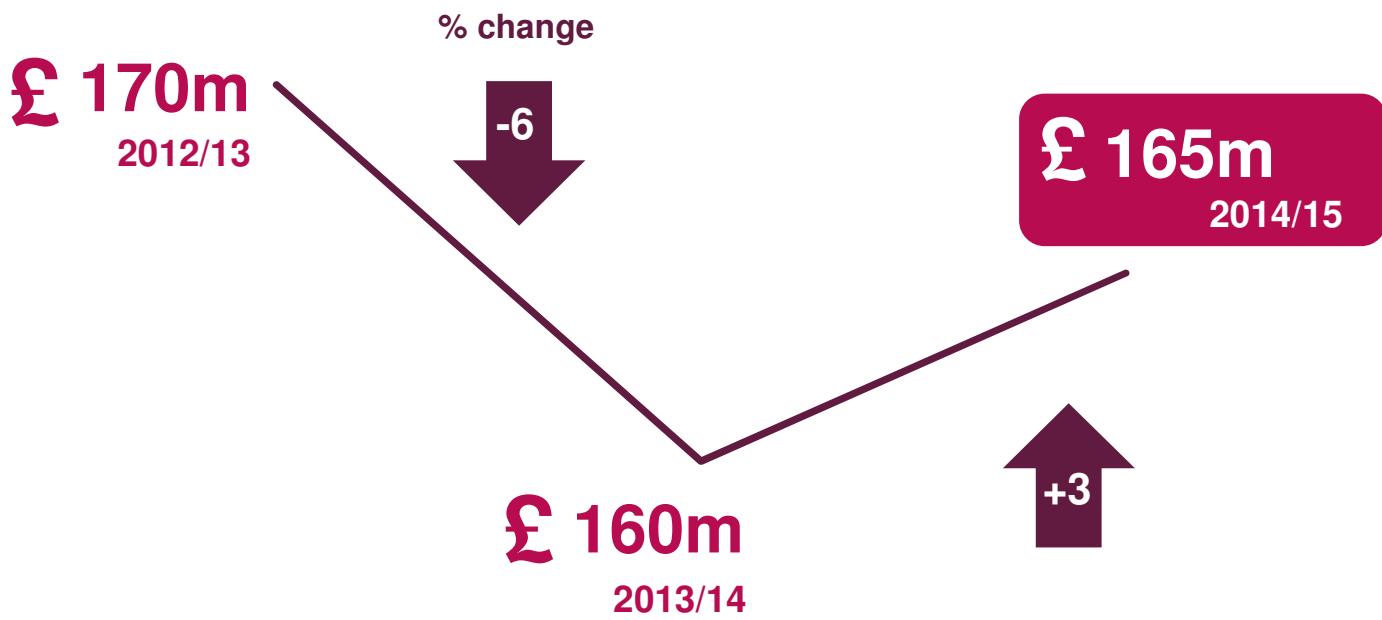

Source: Salford State of the VCSE sector survey 2016/17

Base: 115 All figures are in 2014/15 prices

This change in income should be viewed in the wider national context discussed in Chapter two. The picture is somewhat more positive than in the previous 2012/13 study. Between 2012/13 and 2013/14 the income and spending of the VCSE sector in the UK increased, representing the first notable net growth since the peaks of $2007 / 08$ and 2009/10 respectively. While the data above shows a decrease in Salford between these two years results indicate a more recent upturn in the local area. However, with austerity measures set to continue for the foreseeable future and public sector funding for the sector continuing to be squeezed, there is still need for caution.

When the VCSE sector's income is explored in more detail it shows notable variations according to organisation size ${ }^{17}$. In 2014/15, the majority of income was concentrated in large and medium sized organisations even though the majority of organisations were micro or small. This is outlined in more detail in figure 5.2.

\footnotetext{
${ }^{17}$ In exploring organisation size we used the categories developed by NCVO for use in their Almanac series (see e.g. Clark et al., 2010)
} 
Figure 5.2: Proportion of organisations and proportion of income by organisation size $(2014 / 15)$

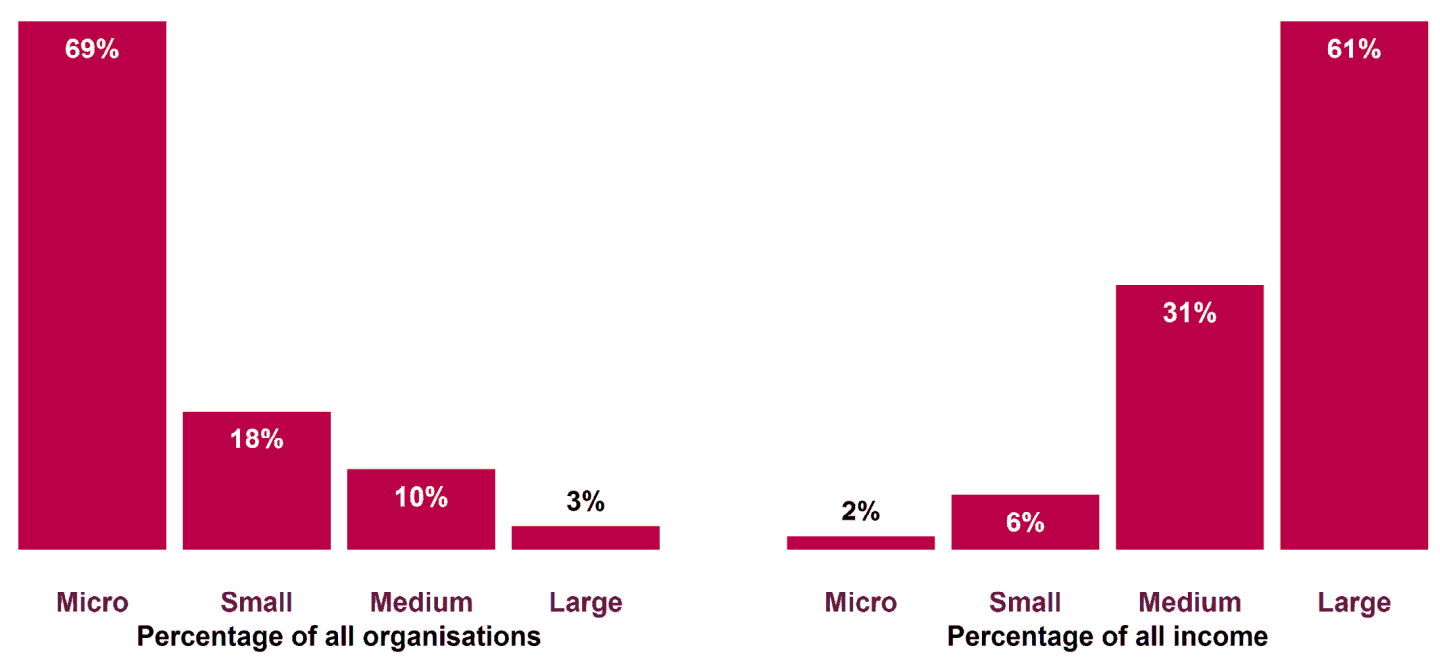

Source: Salford State of the VCSE sector survey 2016/17

Base: 115

This shows that micro and small organisations account for almost nine out of ten organisations in the VCSE sector but less than ten per cent of total income. By contrast medium and large organisations account for just 13 per cent of the VCSE sector's organisations but receive more than 90 per cent of its income. Income is concentrated particularly in the largest organisations, with around three fifths of all income ( 61 per cent) into the VCSE sector received by only 46 organisations.

Analysis of income data from survey respondents across Greater Manchester ${ }^{18}$ identified further variations according to organisation size when we explored how income levels had changed between 2012/13 and 2014/15. These are summarised in table 5.1 .

Table 5.1: Estimated change in annual income by organisation size (all Greater Manchester organisations: 2012/13-2014/15)

\begin{tabular}{|c|c|c|c|c|c|c|c|c|}
\hline & \multicolumn{2}{|c|}{$\begin{array}{c}\text { Micro } \\
\text { (under } £ 10 k \text { ) }\end{array}$} & \multicolumn{2}{|c|}{$\begin{array}{c}\text { Small } \\
(£ 10 k-£ 100 k)\end{array}$} & \multicolumn{2}{|c|}{$\begin{array}{c}\text { Medium } \\
(£ 100 k-£ 1 m)\end{array}$} & \multicolumn{2}{|c|}{$\begin{array}{c}\text { Large } \\
\text { (more than } £ 1 \mathrm{~m})\end{array}$} \\
\hline & Income & $\begin{array}{c}\% \\
\text { change }\end{array}$ & Income & $\begin{array}{c}\% \\
\text { change }\end{array}$ & Income & $\begin{array}{c}\% \\
\text { change }\end{array}$ & Income & $\begin{array}{c}\% \\
\text { change }\end{array}$ \\
\hline $2012 / 13$ & $£ 32.3 \mathrm{~m}$ & & $£ 84.9 \mathrm{~m}$ & & $£ 413.9 \mathrm{~m}$ & & $£ 829.2 \mathrm{~m}$ & \\
\hline $2013 / 14$ & $£ 31.0 \mathrm{~m}$ & -4 & $£ 82.4 \mathrm{~m}$ & -3 & $£ 382.8 \mathrm{~m}$ & -8 & $£ 785.1 \mathrm{~m}$ & -5 \\
\hline 2014/15 & $£ 30.0 \mathrm{~m}$ & -3 & $£ 77.0 \mathrm{~m}$ & -7 & $£ 391.5 \mathrm{~m}$ & 2 & $£ 822.6 \mathrm{~m}$ & 5 \\
\hline
\end{tabular}

Source: Greater Manchester State of the VCSE sector survey 2016/17

Base: 720 All figures are in 2014/15 prices

This shows that across Greater Manchester the micro and small organisation categories experienced year on year reductions in total income between 2012/13 and 2014/15. For micro organisations this is a continuation of a trend identified in the 2012/13 survey where these organisations experienced a reduction of more than 10

\footnotetext{
${ }^{18}$ It was not possible to undertake sufficiently robust analysis of these trends at a local authority level
} 
per cent between 2010/11 and 2011/12. In contrast the 2012/13 survey identified a small increase in income between 2010/11 and 2011/12 for small organisations.

By contrast medium and large organisations saw a reduction in total income between $2012 / 13$ and 2013/14 but then an increase between 2013/14 and 2014/15. For medium organisations this could indicate the start of a reversal in a trend identified in both the 2010 and 2012/13 surveys where year-on-year reductions in income were identified. This income volatility is a significant challenge in the operating context for medium and large organisations.

\subsection{Sources of Income}

\subsubsection{Public sector income}

Survey respondents were asked to identify the public sector bodies from which they received funding in their most recent financial year. Overall, 75 per cent of respondents reported having at least one source of public sector funds. This is higher than both the 62 per cent who reported having public sector funds in the 2012/13 survey and the figure for Greater Manchester as a whole (68 per cent).

Grant funding administered by Salford CVS on behalf of a public sector body was the most frequently identified source of public sector funding (36 per cent). This was a new option added to the 2016/17 survey and may explain the increase in the percentage of respondents reporting having at least one source of public sector funds identified above. The second most common source of public sector funding was Salford City Council (33 per cent) which was the most frequently identified source in 2012/13. One fifth (20 per cent) of organisations also indicated they received funding from NHS Salford Clinical Commissioning Group. Only five per cent or fewer organisations stated they received public sector funding from any of the other public sector bodies listed. Across Greater Manchester local authorities consistently emerged as the most frequent source of public sector funds.

Respondents were also asked to estimate the proportion of their group or organisation's total income that each source of public sector income represented. Figure 5.3 shows the three most common sources of public sector funding received and the estimated proportion of total income this represents.

Figure 5.3 shows that for two fifths (40 per cent) of those receiving grant funding administered by Salford CVS, this represented less than 10 per cent of their total income. At the other end of the spectrum for 10 per cent of organisations this represented at least 75 per cent of their total income.

For those receiving funding from Salford City Council this funding represented less than 10 per cent of their total income for almost two fifths (39\%) of organisations. In contrast for over one quarter (27 per cent) this funding represented at least 75 per cent of income overall.

The survey also asked respondents with public sector income whether they had received a formal funding agreement for each source. Of the three most frequently identified sources, 98 per cent of grant funding administered by Salford CVS on behalf of a public sector body, 88 per cent of Salford City Council funding, and 81 per cent of NHS Salford Clinical Commissioning Group funding, was made with a formal agreement.

Where formal agreements are in place for 35 per cent of organisations at least one of these agreements is on a payment by results basis. 
Figure 5.3: Public sector funds received by Salford respondents (2014/15)

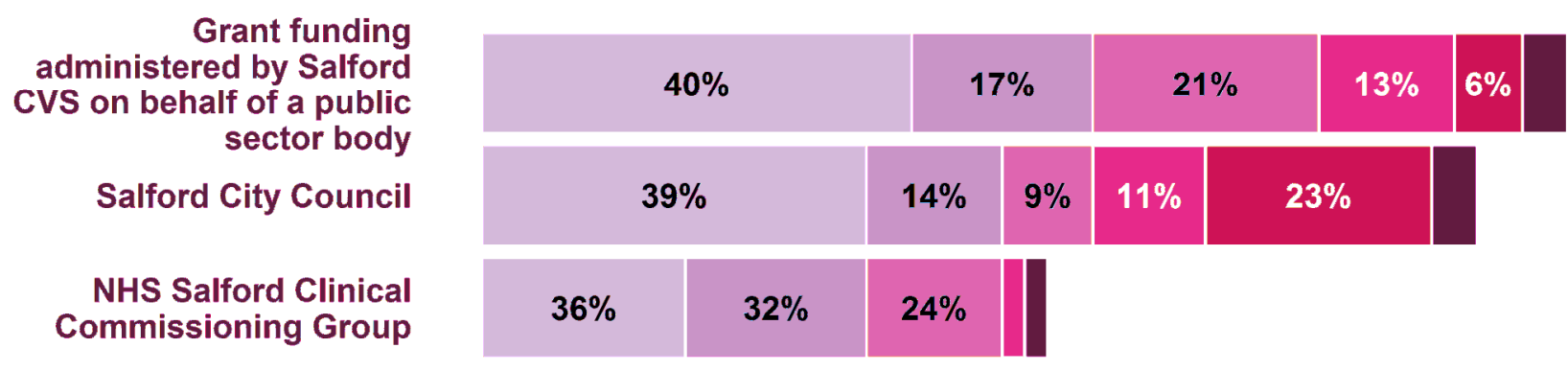

Less than $10 \%$

The survey also revealed notable variations in public sector income received by organisations of different sizes. Similar to 2012/13 and 2010, micro organisations were less likely than small, medium and large organisations to have at least one source of public sector income. This is outlined in more detail in figure 5.4.

This shows that only 63 per cent of micro organisations that responded to the survey received public sector funding compared to 87 per cent of small organisations, 82 per cent of medium organisations and 100 per cent of large organisations. The percentage of small organisations receiving public sector income is 37 percentage points higher than in 2012/13.

Figure 5.4: Proportion of Salford organisations in receipt of public sector funds by organisation size (2014/15)

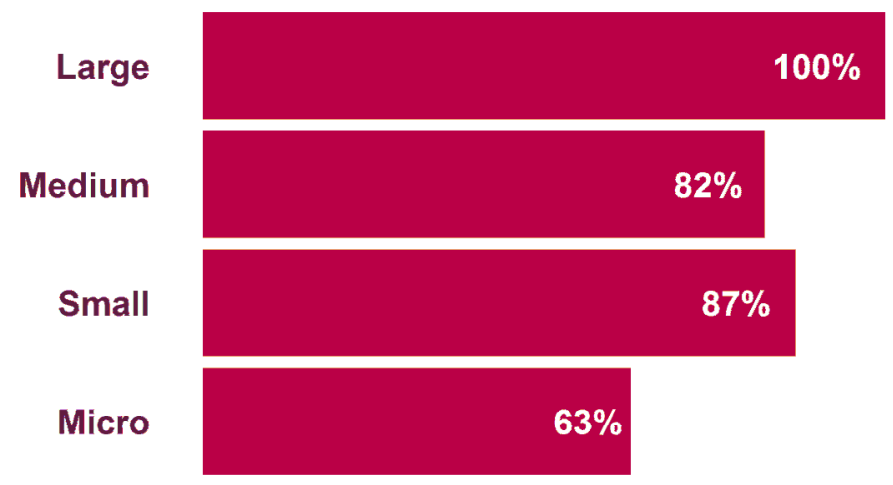

Source: Salford State of the VCSE sector survey 2016/17

Base: 112

\subsubsection{Other sources of income}

Survey respondents were also asked to identify any other sources of income (i.e. non-public sector) they received in 2014/15. Overall, 79 per cent of respondents received funds from at least one non-public sector source. This is decrease of 11 percentage points compared to 2012/13 following an increase of 10 percentage points between 2010 and 2012/13. Across Greater Manchester 84 per cent of respondents received non-public sector income. 
Fundraising was the most frequently identified source of other funds (40 per cent of respondents) followed by membership fees and subscriptions (32 per cent) and grants from charitable trusts and foundations (32 per cent). Fundraising was also the most common type of other funding received in 2012/13 and across Greater Manchester as a whole.

Respondents were also asked to estimate the proportion of their group or organisation's total income received from each of the non-public sector funding sources. Figure 5.5 shows the most prominent sources of non-public sector funding received and the estimated proportion of total income this represents.

Figure 5.5 shows that for over half ( 57 per cent) of those receiving income from fundraising, this funding represented less than 10 per cent of their total income. At the other end of the spectrum for 13 per cent of organisations this represented at least 50 per cent of their total income.

Figure 5.5: Other funds received by Salford respondents (2014/15)

Fundraising (e.g. crowdfunding events, donations etc.)

$$
\begin{array}{r}
\begin{array}{r}
\text { Membership fees I } \\
\text { subscriptions }
\end{array} \\
\text { Grants from charitable trusts } \\
\text { and foundations } \\
\text { Charging for goods and } \\
\text { services } \\
\text { Grants from National Lottery } \\
\text { distributors (e.g. BIG) } \\
\text { Business donations or } \\
\text { sponsorship } \\
\text { Interest (e.g. bank, }
\end{array}
$$
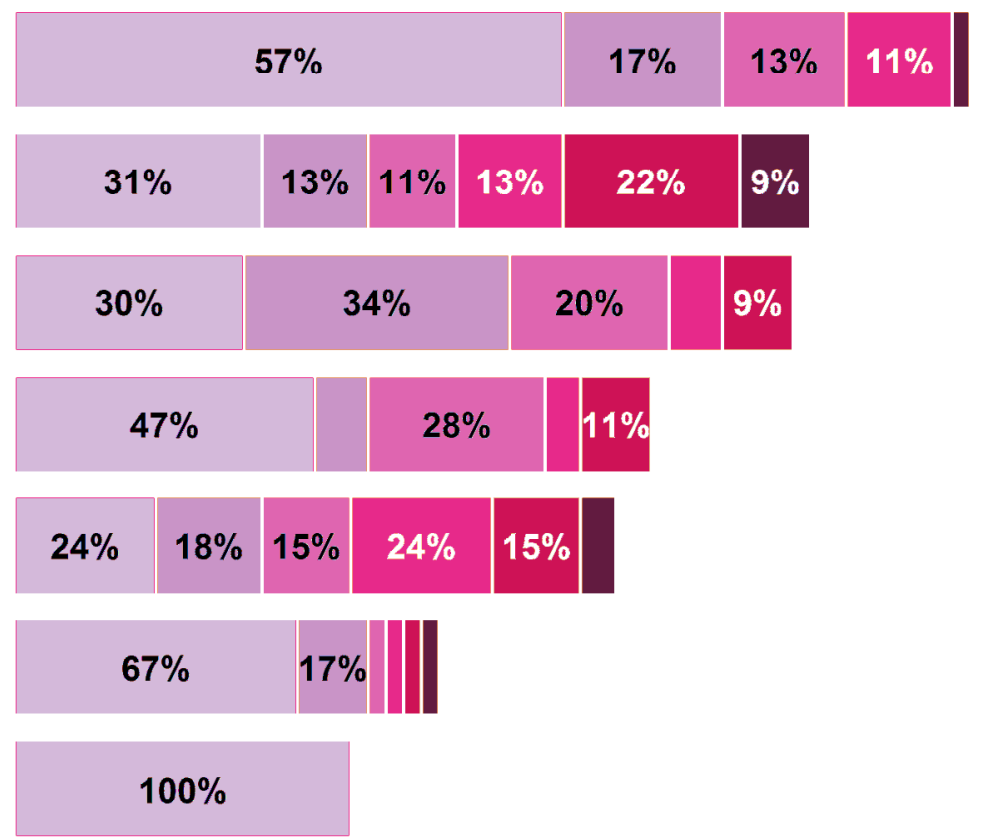

Less than $10 \%$ At least $10 \%$ but
less than $20 \%$ At least $20 \%$ but less than $50 \%$ At least $50 \%$ but
less than $75 \%$ At least $75 \%$ but less than $100 \%$ $100 \%$

Source: Salford State of the VCSE sector survey 2016/17

Base: $19-54$

In line with public sector income, micro organisations were less likely than small, medium and large organisations to have income from non-public sector sources. This is demonstrated by figure 5.6. As in 2012/13 a majority (around three-quarters or more) of each size of organisation had income from non-public sector sources. 
Figure 5.6: Proportion of organisations in receipt of other funds by organisation size (2014/15)

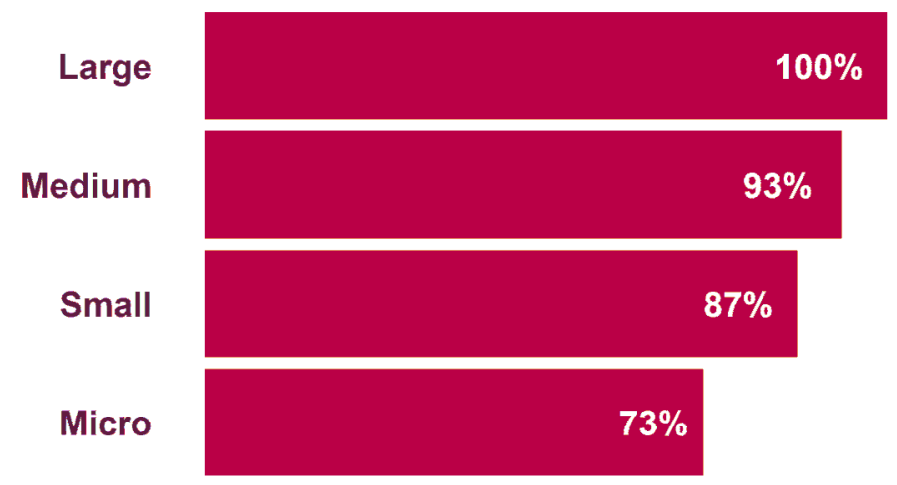

Source: Salford State of the VCSE sector survey 2016/17

Base: 111

\subsection{Financial Sustainability}

The survey asked respondents about how their organisation's financial situation had changed in the past 12 months (i.e. during the current financial year). The results are outlined in figure 5.7 .

Figure 5.7: Change in financial circumstances in the last 12 months

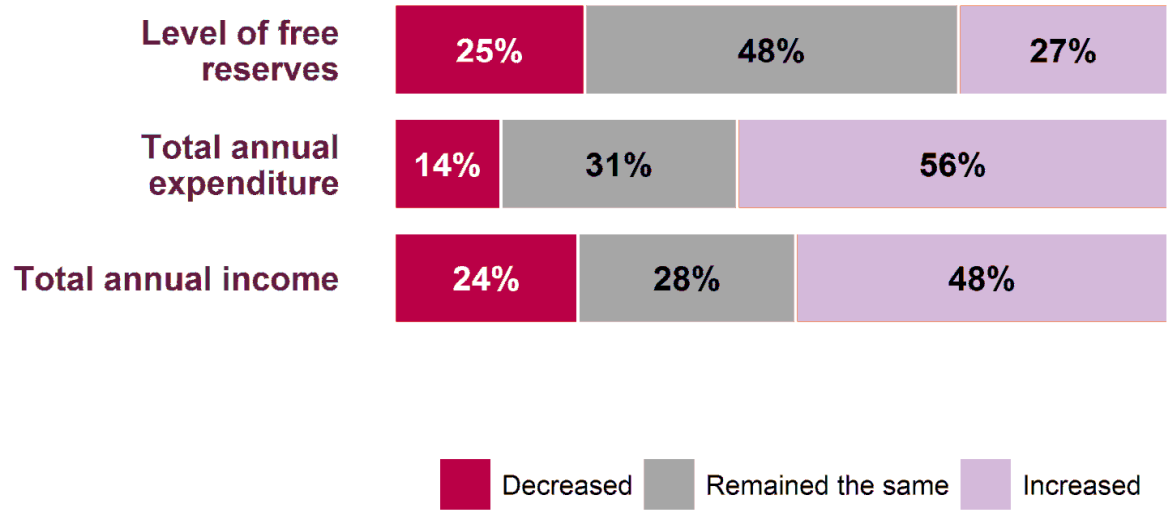

Source: Salford State of the VCSE sector survey 2016/17

Base: 135 (income), 131 (expenditure), 118 (free reserves)

Note: 'cannot say' response has been excluded from the analysis

This raises some concerns: 56 per cent of respondents reported increasing their expenditure but only 48 per cent had experienced an increase in income and only 27 per cent reported an increase in reserves. In addition, 24 per cent of respondents reported a decrease in income but only 14 per cent reduced their expenditure.

21 per cent of respondents provided an expenditure figure for 2014/15 that was greater than their income. This means that there were a notable number of organisations that spent more money than they received in the past 12 months. This figure is, however, lower than the 34 per cent of organisations who provided an expenditure figure in the 2013 study that was greater than their income, nevertheless 
it still appears that the sustainability of a sizeable number of organisations could be under threat.

Explored by organisation size, collectively, the data indicates that the sustainability of medium sized organisations is of particular concern: 71 per cent of medium organisations reported increasing their expenditure in the past 12 months but only 41 per cent increased their income. This is outlined in more detail for all sizes of organisations in figures $5.8 \mathrm{a}$ and $5.8 \mathrm{~b}$ below.

Figure 5.8a: Change in income in the last 12 months by organisation size

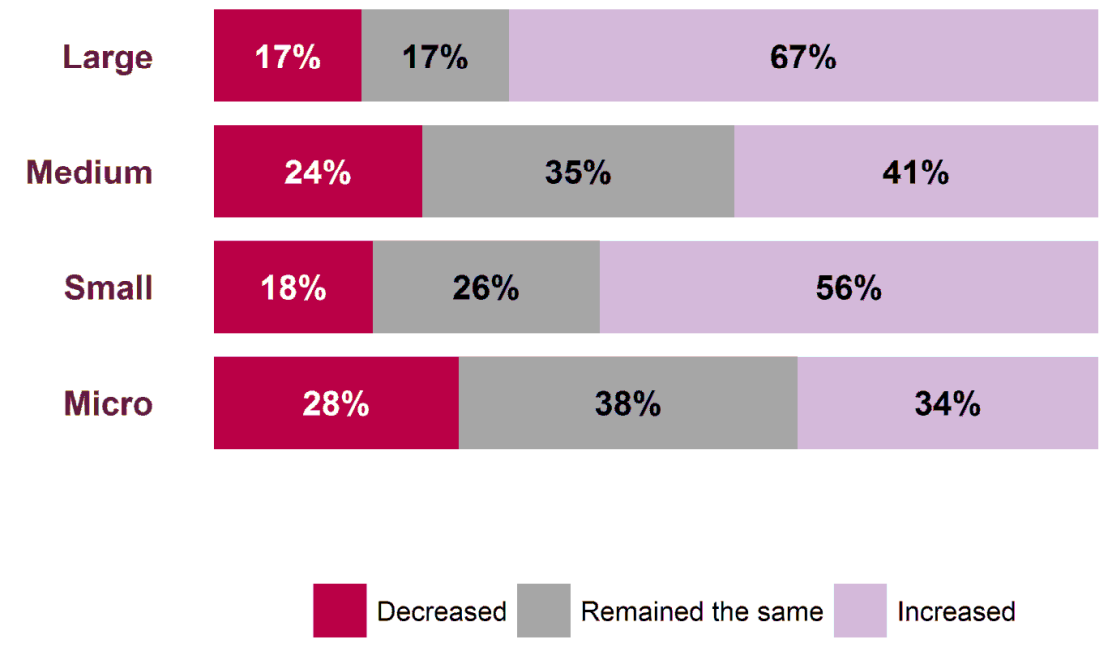

Source: Salford State of the VCSE sector survey 2016/17

Base: 109

Note: 'cannot say' response has been excluded from the analysis

Figure 5.8b: Change in expenditure in the last 12 months by organisation size

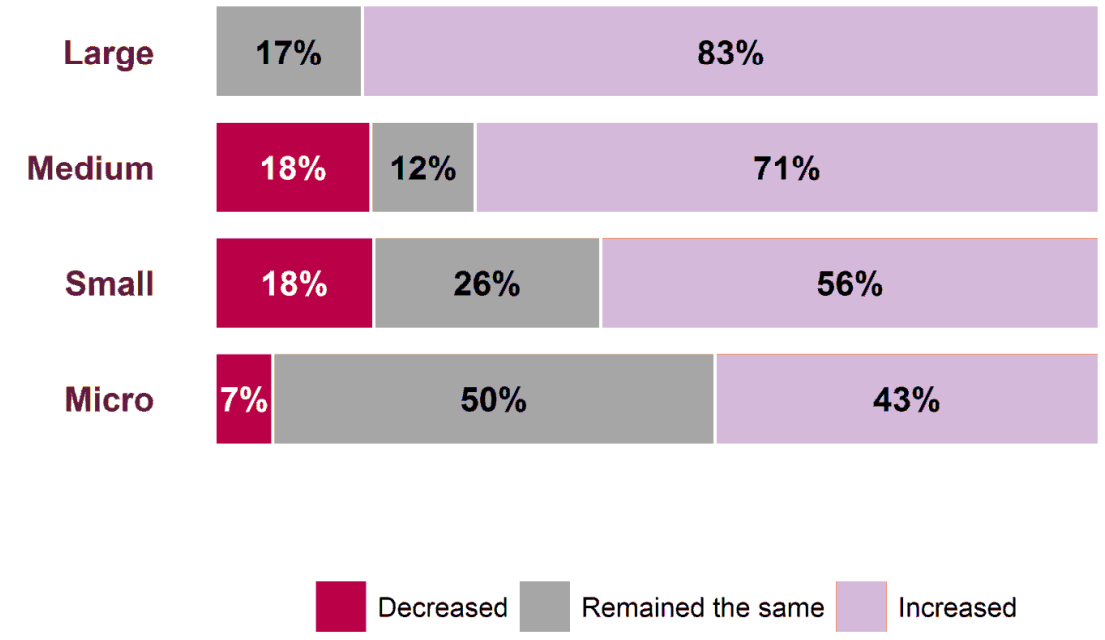

Source: Salford State of the VCSE sector survey 2016/17

Base: 108

Note: 'cannot say' response has been excluded from the analysis

Further analysis of the financial reserve levels reported by respondent organisations provides an additional insight in to the financial health of the VCSE sector. Reserves are important as they provide organisations with funds to fall back on in the short term should other sources of funding reduce or be withdrawn. They also provide 
organisations with the flexibility to develop new and innovative activity that might not have attracted external funding from the outset. Organisations with low reserves relative to expenditure are therefore more likely to be restricted in their ability to adapt if key external funding is lost. In order to explore this issue in more detail reserves (2014/15) were calculated as a proportion of expenditure $(2014 / 15)$ for each respondent. The results are shown in figure 5.9.

\section{Figure 5.9: Financial vulnerability of organisations in Salford}

Less than 1 month expenditure in reserve

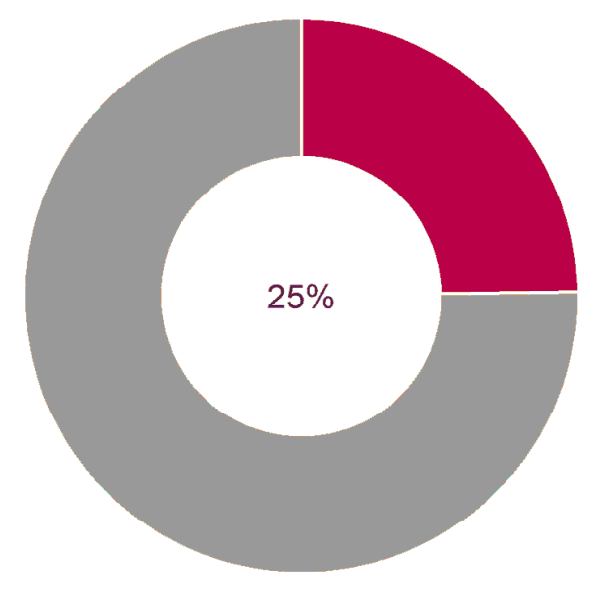

Less than 3 months of expenditure in reserve

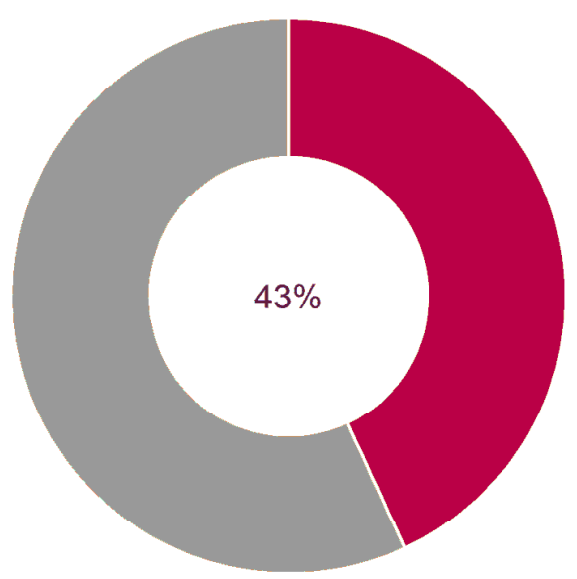

Source: Salford State of the VCSE sector survey 2016/17

Base: 109

This shows that 25 per cent had reserve levels of less than one month's expenditure, and a further 18 per cent had reserves that covered less than three month's expenditure. This suggests that over two-fifths of all organisations in the VCSE sector could be vulnerable should their funds be severely reduced or withdrawn. This is in line with 2012/13 survey when 40 per cent of organisations reported reserves equalling less than three month's expenditure and very similar to the pattern identified at the Greater Manchester level.

Survey respondents were also asked how they thought the environment for funding/income for the VCSE sector will change over the next year. Figure 5.10 shows the responses received to this question. This shows that over two fifths (43 per cent) of organisations in Salford thought the environment will deteriorate compared to just 12 per cent who felt the environment is set to improve. One fifth saw the environment for funding/income staying the same. These results were slightly more positive than across Greater Manchester as a whole where 56 per cent thought the environment will deteriorate and just seven per cent saw the environment improving. 
Figure 5.10: Change in the environment for funding/income in the next year

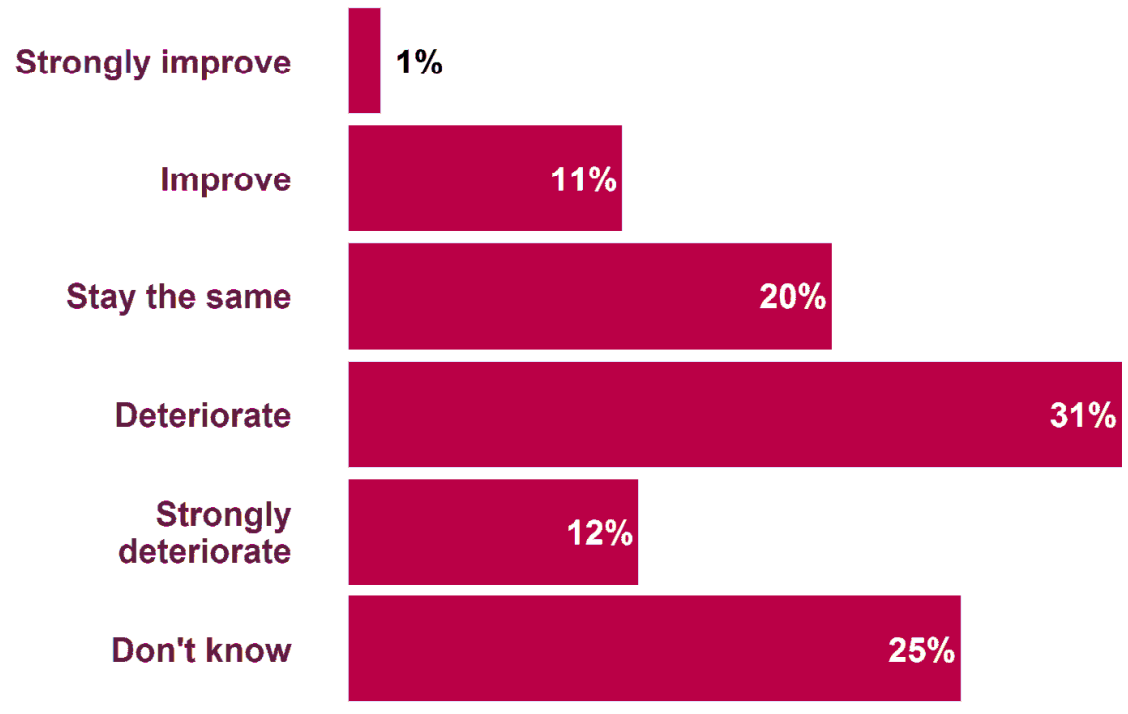

Source: Salford State of the VCSE sector survey 2016/17

Base: 153 


\section{Paid Employees}

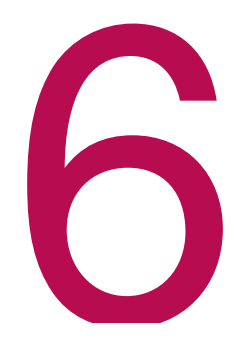

This chapter looks at the paid workforce of the VCSE sector in Salford.

\subsection{How many FTE paid staff are employed in the VCSE sector in Salford?}

Based on the average number of FTE paid staff employed by organisations responding to the survey across Greater Manchester, and drawing on the assumptions used to estimate the total number of organisations in Salford, it is estimated that:

\section{3,500 FTE paid staff were employed in the VCSE sector in Salford in 2016/17}

This represents 5,300 employees.

This was 12 per cent of the estimated total number of FTE paid staff working within the VCSE sector in Greater Manchester.

This is a lower figure than the 3,800 FTE paid staff estimated to work in the sector in the 2013 study.

Gross Value Added (GVA), the value of goods and services produced, is a key measure of the economic contribution of organisations or sectors. It can be estimated for paid employees working in Salford organisations by multiplying the number of FTE paid staff by the estimated gross value added (GVA) per FTE employee ${ }^{19}$. From this calculation it is estimated:

\section{$£ 111.2 \mathrm{~m}$ contributed to the economy per annum by paid employees of Salford VCSE sector organisations}

Over half (55 per cent) of FTE paid staff were employed in large organisations with an income of at least one million pounds. In comparison the 1,309 micro and small organisations employed just 13 per cent of FTE paid staff. The remaining 31 per cent were employed in medium sized organisations. As in 2012/13, large and medium sized organisations especially continue to be significant employers in Salford.

\footnotetext{
${ }^{19}$ This study used Greater Manchester GVA per employee averaged across the following two VCSE sectors: education and human health and social work activities.
} 
Figure 6.1 presents a breakdown of responding organisations by the number of FTE paid staff they employed. Just over four fifths (81 per cent) of organisations employed less than five FTE paid staff members. Included in this figure were 56 per cent of organisations that did not employ any paid staff. Further analysis reveals that the majority of these were micro organisations with income of less than $£ 10,000$. At the other end of the spectrum seven per cent of organisations employed 20 or more FTE paid members of staff. This pattern is broadly equivalent to that identified in both the $2012 / 13$ and 2010 surveys.

Compared with the Greater Manchester sample as a whole, a lower proportion of organisations within Salford appeared to have FTE paid staff: 44 per cent in Salford compared with 49 per cent in Greater Manchester.

Figure 6.1: Organisations by numbers of FTE paid staff

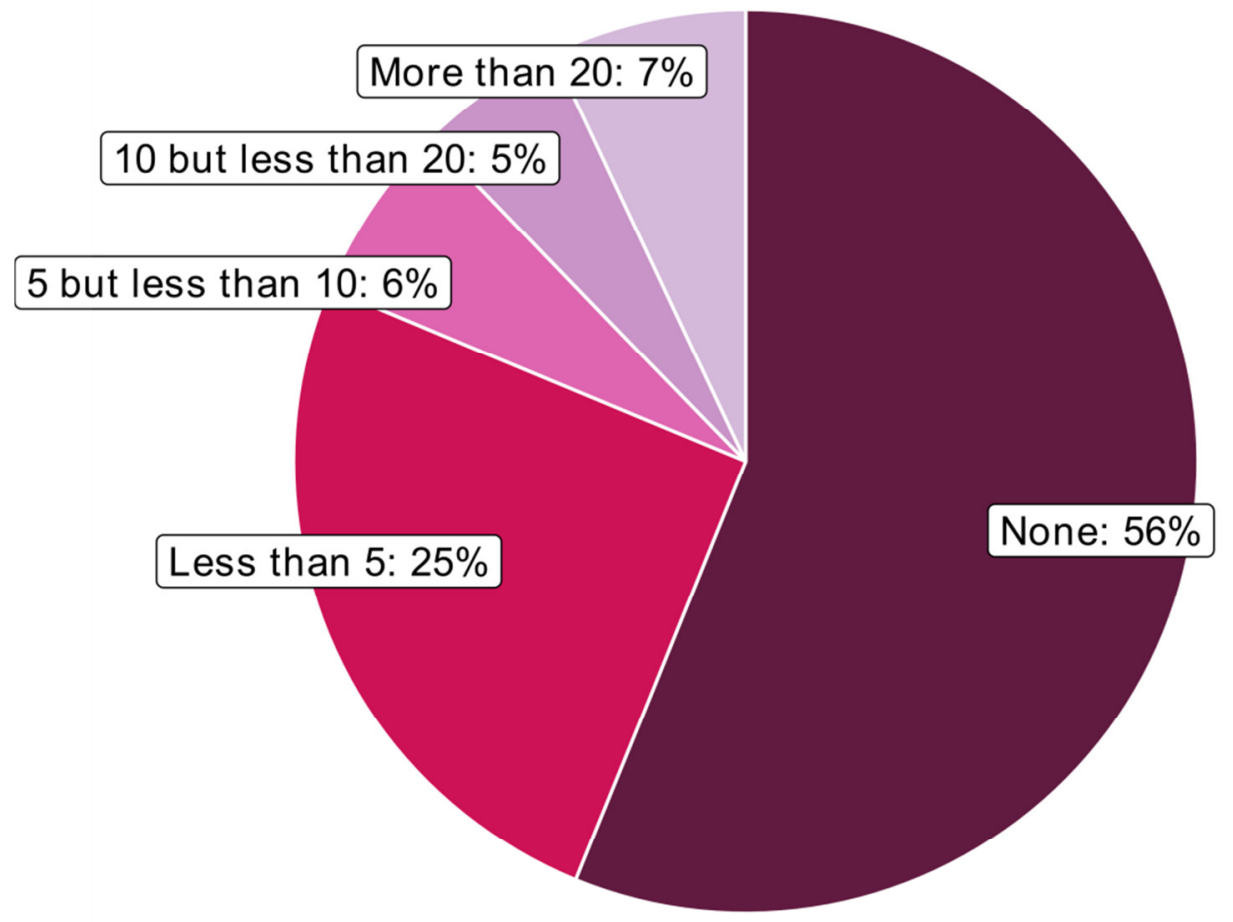

Source: Salford State of the VCSE sector survey 2016/17

Base: 171

\subsection{How has the VCSE sector's workforce changed in the last 12 months?}

The survey asked respondents whether the number of staff in their organisation's workforce had 'increased', 'remained the same' or 'decreased' in the last year compared to the previous year. Figure 6.2 presents the results to this question, the key findings of which are:

\section{Paid employees:}

- 67 per cent of organisations employed a similar number of paid employees compared to a year ago

- 23 per cent of organisations reported an increase in paid staff which was over double the percentage that reported a decrease (10 per cent)

- an equivalent percentage of organisations reporting a change in 2012/13, reported an increase in paid staff as a decrease ( 21 per cent respectively) while 
in 2010,28 per cent reported an increase in the past year which was double the percentage that reported a decrease (14 per cent)

- $\quad$ across Greater Manchester 22 per cent of organisations reported an increase in their number of paid employees, while 16 per cent reported a decrease.

Figure 6.2: Change in aspects of the workforce (paid staff) in the last 12 months

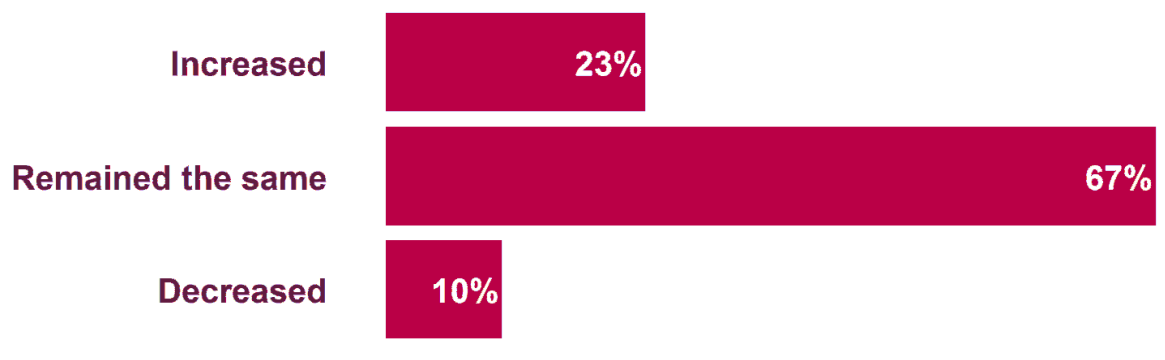

Source: Salford State of the VCSE sector survey 2016/17

Base: 145

Note: 'cannot say' response has been excluded from the analysis 


\section{Volunteers}

This chapter looks at the volunteers within the VCSE sector in Salford.

7.1. How many volunteers are part of the VCSE sector workforce in Salford and what is their economic contribution?

Based on responses to the survey across Greater Manchester on questions exploring the numbers of volunteers and committee/board members and the hours which they contribute, and drawing on the assumptions used to estimate the total number of organisations in Salford, it is estimated there are:

\section{6,800 volunteers or committee/board members in the VCSE sector's workforce in Salford in 2016/170}

This includes:

\section{6,500 volunteers in the VCSE sector's workforce in Salford in 2016/17}

\section{0,400 committee/board members in the VCSE sector's workforce in Salford in 2016/17}

This figure for volunteers represents 15 per cent of Salford's total population $(245,600)$ and 10 per cent of the estimated total for Greater Manchester organisations.

It is also estimated that:

\section{5,400 hours of their time provided by these volunteers and committee/board members per week}

This represents 11 per cent of the estimated number of volunteer and committee/board member hours for all Greater Manchester organisations.

\footnotetext{
${ }^{20}$ It is possible in cases where a person is volunteering for more than one organisation they could have been counted more than once; additionally, there will be residents from outside of Salford volunteering within Salford; and conversely there will be Salford residents volunteering for organisations outside of Salford
} 
The $2012 / 13$ study estimated there were 37,300 volunteers in Salford who provided 137,000 hours per week. The previous study did not ask for volunteers and committee/board members to be recorded separately so caution should be applied when making comparisons.

There are two broad approaches to valuing the contribution of volunteers. One method, and this study's preferred approach, is to value the output that they produce. In effect this is the value to society of the goods and services that volunteers produce. This can be estimated by multiplying the number of FTE volunteers by the estimated gross value added (GVA) per FTE employee. ${ }^{21}$

From this calculation:

\section{$£ 104.4$ million per annum estimated as the economic contribution of volunteers and committee/ board members in Salford organisations}

The use of estimated GVA per FTE employee to measure the value of the output produced by volunteers assumes that paid employees would not be used in the absence of volunteers to produce the same level of goods and services. In such a situation the value of output is the value of the labour input (wages and benefits) plus the value of the capital input (for example office space and computers). If paid employees would be used to produce the same level of goods and services then the value of capital input would be borne whether or not volunteers were used. Therefore the value of the output from volunteers would be just the value of the labour input. This value would be roughly equivalent to the value estimated from the input method of valuation which is outlined in the next paragraph.

In the second method, the value of the input of volunteers is used to value the contribution of volunteers ${ }^{22}$. This is the amount that it would cost to pay employees to do the work carried out by volunteers. As such, this can be considered to be the benefit to organisations ${ }^{23}$. However, this benefit might also be passed onto society via lower prices for goods and services due to lower costs of production. The input value of volunteers can be calculated by multiplying the number of hours that volunteers give per week by an estimate of how much it would cost to employ someone to do that work. There are a number of widely accepted hourly rates that could be used to estimate this value; these include: the national minimum wage or national living wage, the local median wage, the local mean wage and the reservation wage. The preference in this study has been to provide a range using the national living wage (low estimate) and the local median wage (high estimate). In reality the true value of the input provided by volunteers will lie between the two estimates. It is estimated that:

- $\quad$ assuming the national living wage for adults ${ }^{24}$ it would cost $£ 43.2$ million annually to employ staff to do the work provided by volunteers in Salford organisations

\footnotetext{
${ }^{21}$ This study used Greater Manchester GVA per employee averaged across the following two VCSE sectors: education and human health and social work activities.

22 This is the approach recommended by Volunteering England

${ }^{23}$ This assumes that there are no additional costs faced by organisations in using volunteers: for example extra management costs

${ }^{24} £ 7.20$ for 25 years and older in 2016
} 
- $\quad$ assuming the median gross hourly wage for full time employees in Greater Manchester ${ }^{25}$ it would cost $\mathfrak{£ 7 7 . 2}$ million annually to employ staff to do the work provided by volunteers in Salford organisations.

Figure 7.1 presents a breakdown of responding organisations by the number of volunteers that they use. Six per cent of respondents indicated they had no volunteers. This is in contrast to the $2012 / 13$ survey when no organisation reported having no volunteers but lower than in 2010 when 12 per cent reported no volunteers. The same proportion of organisations had 50 or more volunteers in the latest survey and in 2012/13 (13 per cent).

This pattern was largely representative of the picture for organisations across Greater Manchester as a whole.

Figure 7.1: Organisations by numbers of volunteers

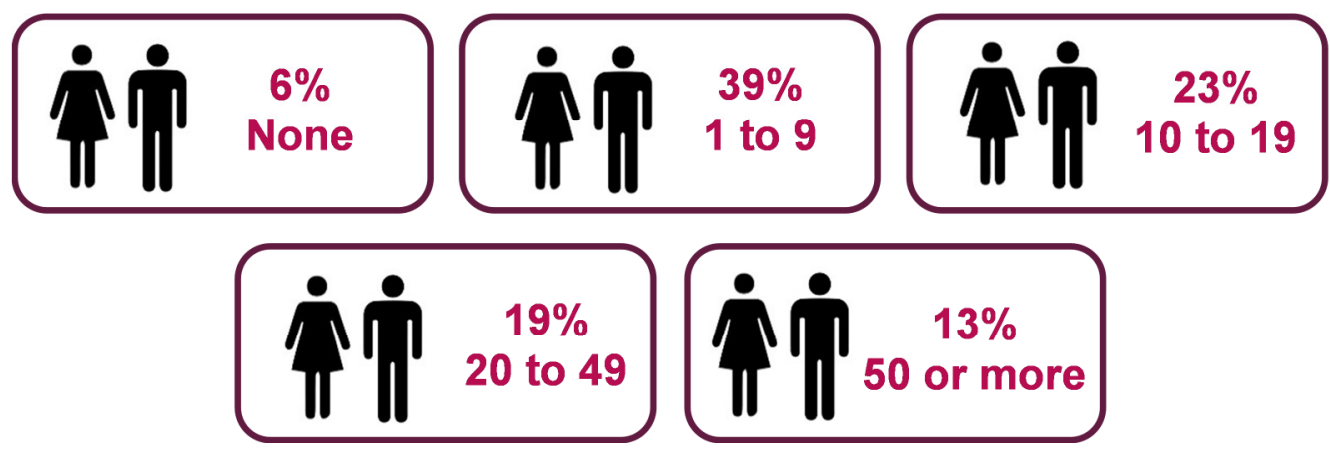

Source: Salford State of the VCSE sector survey 2016/17

Base: 192

\subsection{How has the VCSE sector's workforce changed in the last 12 months?}

The survey asked respondents whether the number of volunteers in their organisation's workforce had 'increased', 'remained the same' or 'decreased' this year compared to the previous year. Figure 7.2 presents the results to this question, the key findings of which are:

- 39 per cent of respondents reported increased numbers of volunteers now compared to a year ago

- in comparison 12 per cent of organisations reported a decrease in volunteer numbers.

The 2012/13 survey found similar results, suggesting the number of volunteers within the VCSE sector has grown consistently over recent years:

- 36 per cent of respondents reported increased volunteer numbers

- about half (49 per cent) reported that volunteer numbers remained the same

- $\quad$ while 15 per cent reported that numbers of volunteers decreased.

Just over two fifths (42 per cent) of Greater Manchester organisations reported an increase in their number of volunteers over the previous year, compared with 13 per cent who reported a decrease.

${ }^{25} £ 12.86$ for 2016 
Figure 7.2: Change in aspects of the workforce (volunteers) in the last 12 months

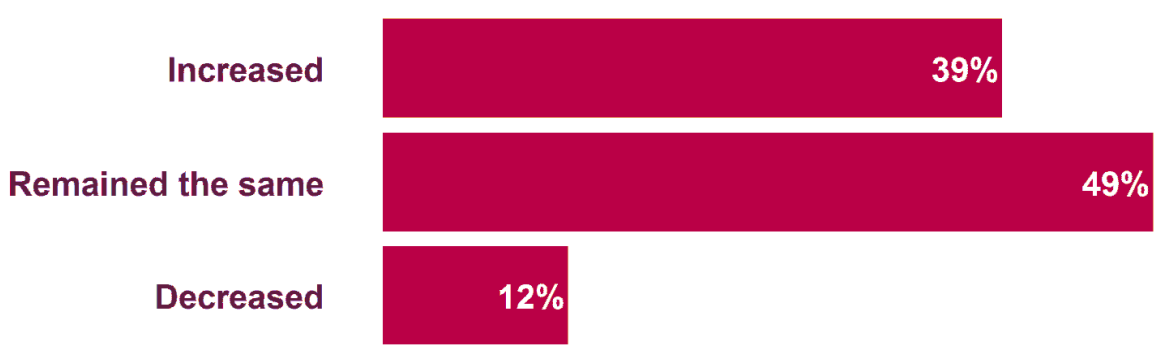

Source: Salford State of the VCSE sector survey 2016/17

Base: 168

Note: 'cannot say' response has been excluded from the analysis

\subsection{Qualitative responses on volunteering}

Focus group participants from registered charities and small VCSE organisations were asked to discuss changes associated with volunteering in recent years. There was a mixed picture in terms of the number of volunteers: for many organisations of all types and sizes numbers had remained relatively unchanged, but some reported increases and others reported reductions.

"We use many volunteers. But I don't think there is a significant increase or decrease. Once we get them, we do keep them."

"The number of volunteers has been reduced because of funding cuts and we also have less time to work with them and really recruit and support them."

"For us, volunteering has increased. We have 10 volunteers and I would say ages range between mid-30s to 60 s and they are all basically assisting with the classes."

"I don't think that it has changed significantly. We still have the same number of inquiries, but what I have seen is that with the employability agenda, we have seen more people coming in to get their work trials; so the flow peaks at certain times and the challenge has been to manage these expectations."

"We have a steady flow, but in the old days we could have a volunteer coordinator and we could spend more time in supporting them, training them, and now there is no funding for that anymore."

Volunteering was regarded by focus group participants as a core and unique feature of their work with a range of benefits for the organisations. Volunteers brought new skill-sets and fresh ideas; enabled new services to be developed and existing services to be improved; improved organisational democracy; increased the visibility of the organisation in the community; and raised diversity and cultural awareness, ensuring accountability and staff responsibility. Participants from small voluntary groups in particular highlighted the role of volunteers as role models of active citizenship, arguing that their efforts have a positive snowballing effect with other people in the community being inspired by them, becoming volunteers themselves, and so on.

"We've done some different work using volunteers which we wouldn't have done; actually couldn't have done without them and got into areas which we wouldn't be 
able to reach. It's also a great route to this service for people who want to do that kind of work."

"We have been able to setup successful programmes just from the support of the volunteers. We have established ourselves more strongly in the community; we have covered skill-gaps; we have been greatly improved by them in areas such as communication, marketing, IT, PR, photography, etc., I can't stress their importance enough."

"For me volunteers bring this added value which you can't really quantify; it's the rapport they can build with service-users, for example. That's huge for us; they are absolutely invaluable."

Participants also identified a number of challenges associated with volunteering that had affected their organisations in recent years. In particular they consistently argued that recruiting volunteers had become a costly and demanding process: the number of volunteers is not the issue most of the time, but the 'quality' and 'type' of volunteers. Increasingly volunteer roles required a much broader and higher level skill-set. This combined with the absence of any funding or support by the public sector had made it very difficult to be able to effectively and consistently screen, train, support, coordinate and incorporate volunteers in an organisation's work.

"For us, sometimes there is a mismatch between what volunteers want to do and the nature of what we need from volunteers. We need people with higher skills and in the centre, we have many people coming in just to do nice things towards others, which is fine, but what we really need is very skilled, very committed volunteers who will give many hours per week to the organisation and it's hard to manage that."

The lack of funds and resources to handle DBS screening and other administrative tasks was a major concern of the participants. Ideally, these should be handled by a volunteer manager or co-ordinator within the organisations but many did not have the resources to cover these roles when the priority was frontline service delivery.

"In our organisation, we have a problem taking new volunteers on. Many problems with the application forms and references, as we don't have a volunteer coordinator. For example, we've had problems with funding bids when they asked us how many volunteers are from Salford and we didn't know, so we need a database but can't pay anyone to make it."

A final challenge identified by participants was the impact of the employability agenda which placed growing emphasis on volunteering as a condition of benefit entitlements. Consequently, many VCSE organisations reported sometimes being 'flooded with volunteers' with limited understanding of what volunteering entailed and the level of commitment required.

"Exactly, and l've also found that for us it's hard to manage these people since when we do get volunteers, we spend so much time training them and then you lose them and the energy you put into that detracts from running the organisation. We need people who are IT-literate, who can organise events, who can fund-raise, are capable in bid-writing and so on. So you ask them to work on a bid, and then they come back and say this is too hard and they get frustrated and that's how you lose them."

The numbers of young people, particularly students, was also reported to have increased in recent years but was not seen as particularly problematic; with many 
participants highlighting benefits in terms of the ideas and enthusiasm they brought to the role.

"We have lots of younger volunteers, we have many students and for about a year now, we have no paid staff whatsoever which I think is a fantastic place to be as a voluntary organisation, when you manage to bring in and keep people with skills and goals who want to volunteer." 


\section{Partnership Working: the Public Sector}

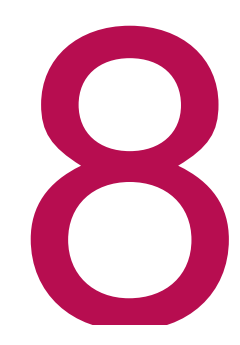

This chapter considers the relationship between the VCSE sector and the public sector, exploring organisations experiences of partnership working with Salford City Council and other public sector bodies.

\section{1. $\quad$ Dealings with local public sector bodies}

Survey respondents were asked about the extent of their dealings with each of the main public sector bodies covering the city of Salford. An overview of their responses is provided in figure 8.1, along with the local authority figure for Greater Manchester combined.

This shows that survey respondents had dealings with a range of local public sector bodies. The three most prominent were Salford City Council, Salford schools and Salford University:

- Salford City Council: 78 per cent had some dealings with the Council; including 14 per cent who had a 'great amount' of dealings and 32 per cent who had a 'fair amount' of dealings

- Salford schools: 58 per cent had some dealings with Salford schools; including 10 per cent who had a 'great amount' of dealings and 14 per cent who had a 'fair amount' of dealings

- University of Salford: 50 per cent had some dealings with the University; including four per cent who had a 'great amount' of dealings and 17 per cent who had a 'fair amount' of dealings.

Salford City Council was also the organisation respondents had the most dealings with in the 2012/13 survey (77 per cent had some dealings) while a similar proportion in 2012/13 had dealings with the University of Salford when compared to the latest survey (46 per cent). Salford schools were a new option added in 2016/17.

Local authorities consistently emerged as the most prominent public sector contact for respondents to this study across Greater Manchester. Overall, 16 per cent of respondents said they had a 'great amount' of dealings with their local authority and 36 per cent said they had a 'fair amount'. 
Figure 8.1: Dealings with local public sector bodies

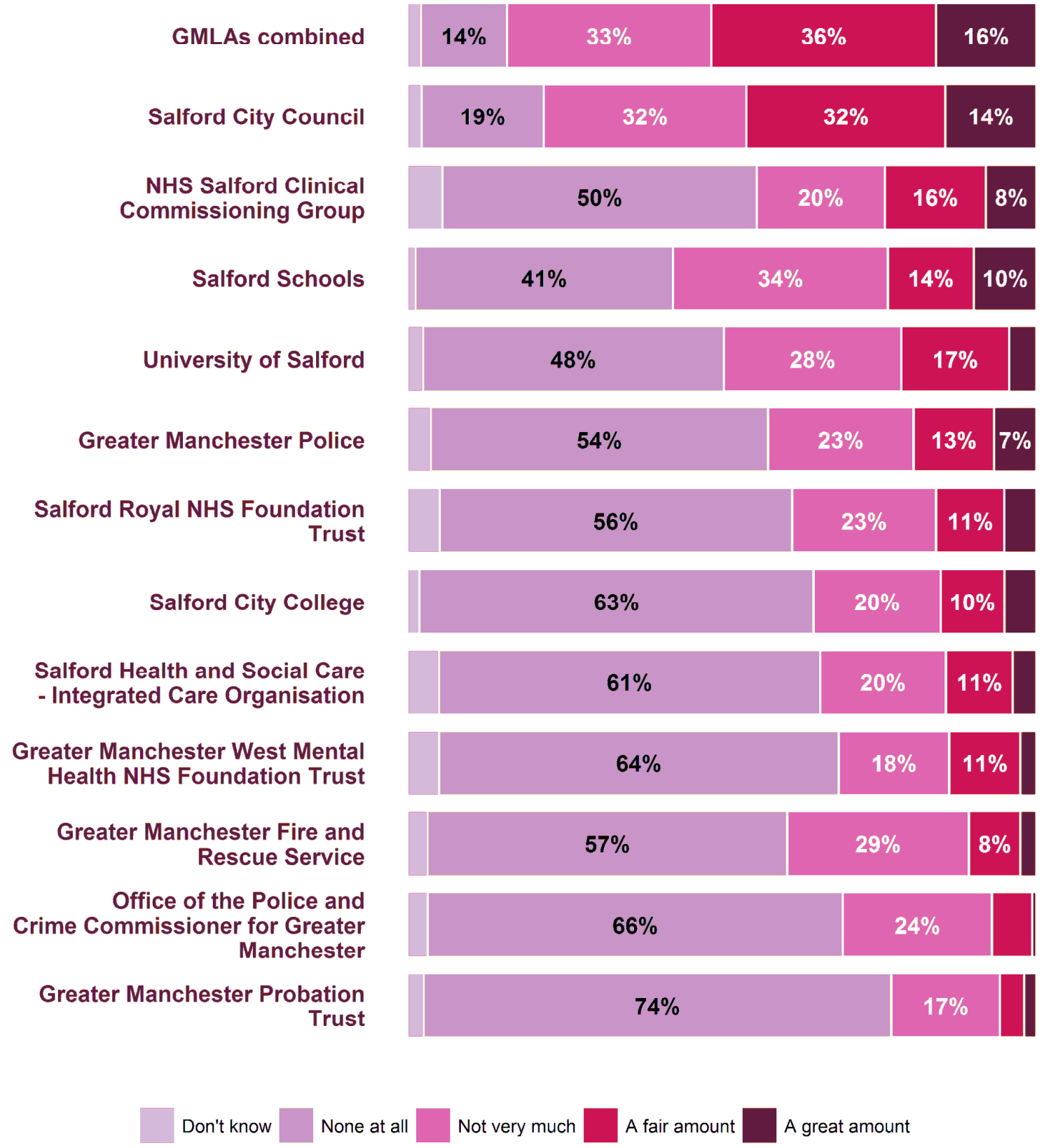

Source: Salford / Greater Manchester State of the VCSE sector survey 2016/17

Base: 156-180 (Salford), 1,080 (Greater Manchester)

Survey respondents were asked to indicate their most frequent public sector contact other than their local authority. Figure 8.2 shows the responses received to this question with Salford schools the most commonly cited followed by, NHS Salford Clinical Commissioning group, the University of Salford and Salford Health and Social Care - Integrated Care Organisation. 
Figure 8.2: Most frequent public sector contact other than local authority

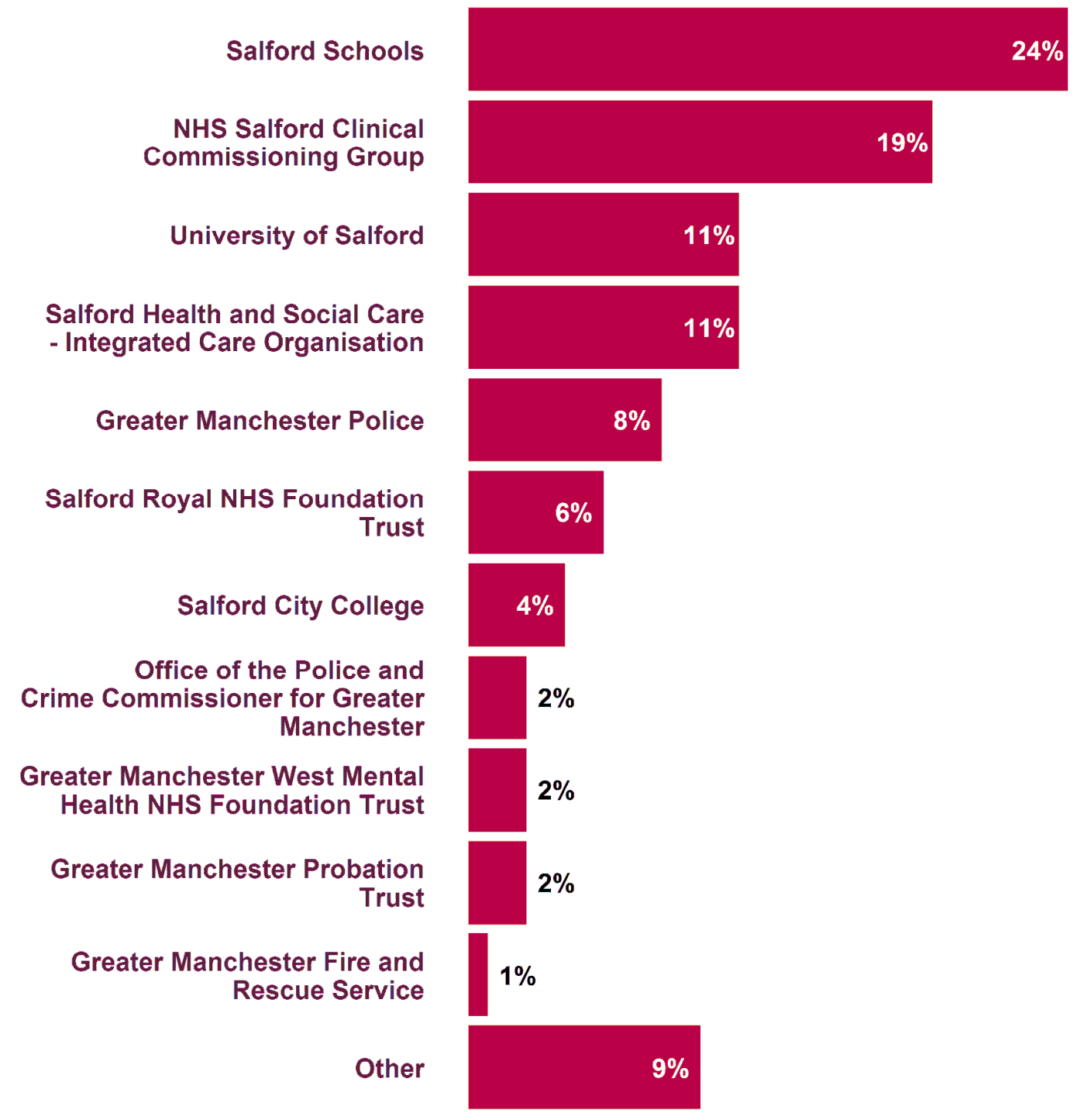

Source: Salford State of the VCSE sector survey 2016/17

Base: 127

Respondents were also asked to consider the extent to which their organisation has direct dealings with any emerging Greater Manchester structures (e.g. Greater Manchester Combined Authority, The Office of the Police and Crime Commissioner, The Health and Social Care Devolution Team etc.). Figure 8.3 presents the results to this question.

Just over one third (35 per cent) had some dealings with any of the emerging structures including just two per cent who had a 'great amount' of dealings and six per cent who had a 'fair amount' of dealings. Results were similar across Greater Manchester where 38 per cent had some dealings, including two per cent who had a 'great amount' of dealings and 10 per cent who had a 'fair amount' of dealings. 
Figure 8.3: Dealings with emerging Greater Manchester structures

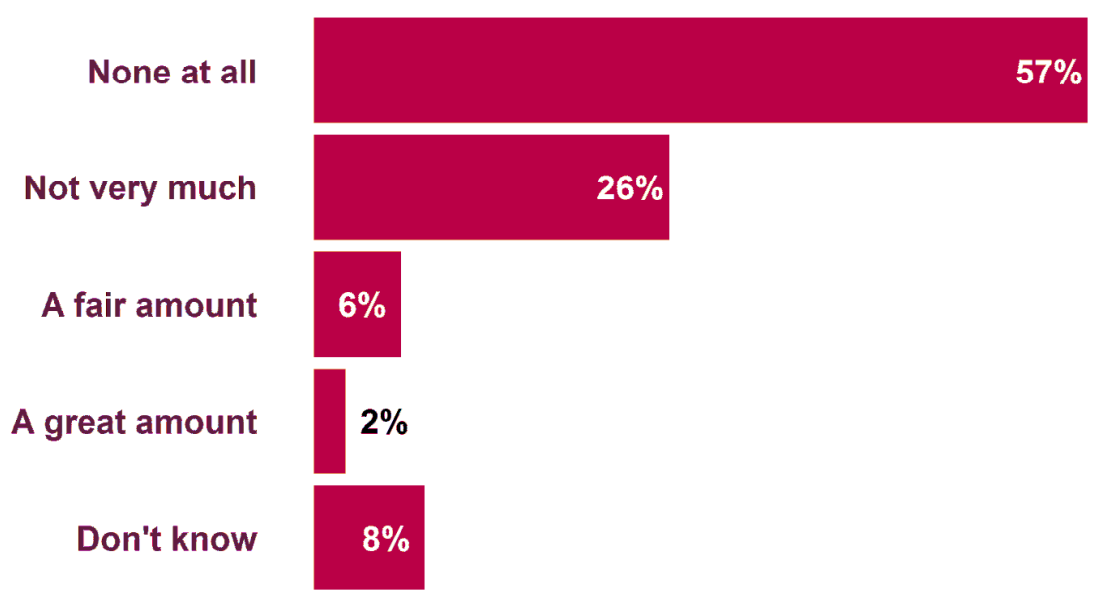

Source: Salford State of the VCSE sector survey 2016/17

Base: 172

\subsection{Relationships with local public sector bodies}

Survey respondents were also asked two further questions about the extent to which their organisations were satisfied with their ability to influence public sector decisions of relevance to their organisation and the extent to which they thought local statutory bodies influenced their success ${ }^{26}$. The results of these questions are summarised in figure 8.4. A comparison with the Greater Manchester average is also provided.

Figure 8.4 shows that 29 per cent of respondents were satisfied with their ability to influence Salford City Council decisions of relevance to their organisation and 35 per cent said that the council had a positive influence on their organisation's success. Results are similar to the Greater Manchester combined figures (30 per cent were satisfied with their ability to influence their local authority and 38 per cent agreed their local authority has a positive influence on their success). Results are more positive than in 2012/13 when just 16 per cent of respondents were satisfied with their ability to influence Salford City Council decisions of relevance to their organisation and 35 per cent said that the council had a positive influence on their organisation's success.

In addition, 41 per cent of respondents said they were satisfied with their ability to influence the key decisions of their most frequent other public sector contact and 57 per cent said this contact had a positive influence on their success. These are slightly higher than the Greater Manchester combined figures (36 per cent and 51 per cent respectively).

\footnotetext{
${ }^{26}$ This latter measure was used in 2008 and 2010 to provide evidence of local authority performance against 'National indicator 7: the environment for a thriving third sector'.
} 
Figure 8.4: Proportion of organisations who said they were satisfied with their ability to influence public sector decisions of relevance to their organisation and who said local public sector bodies influence their organisation's success

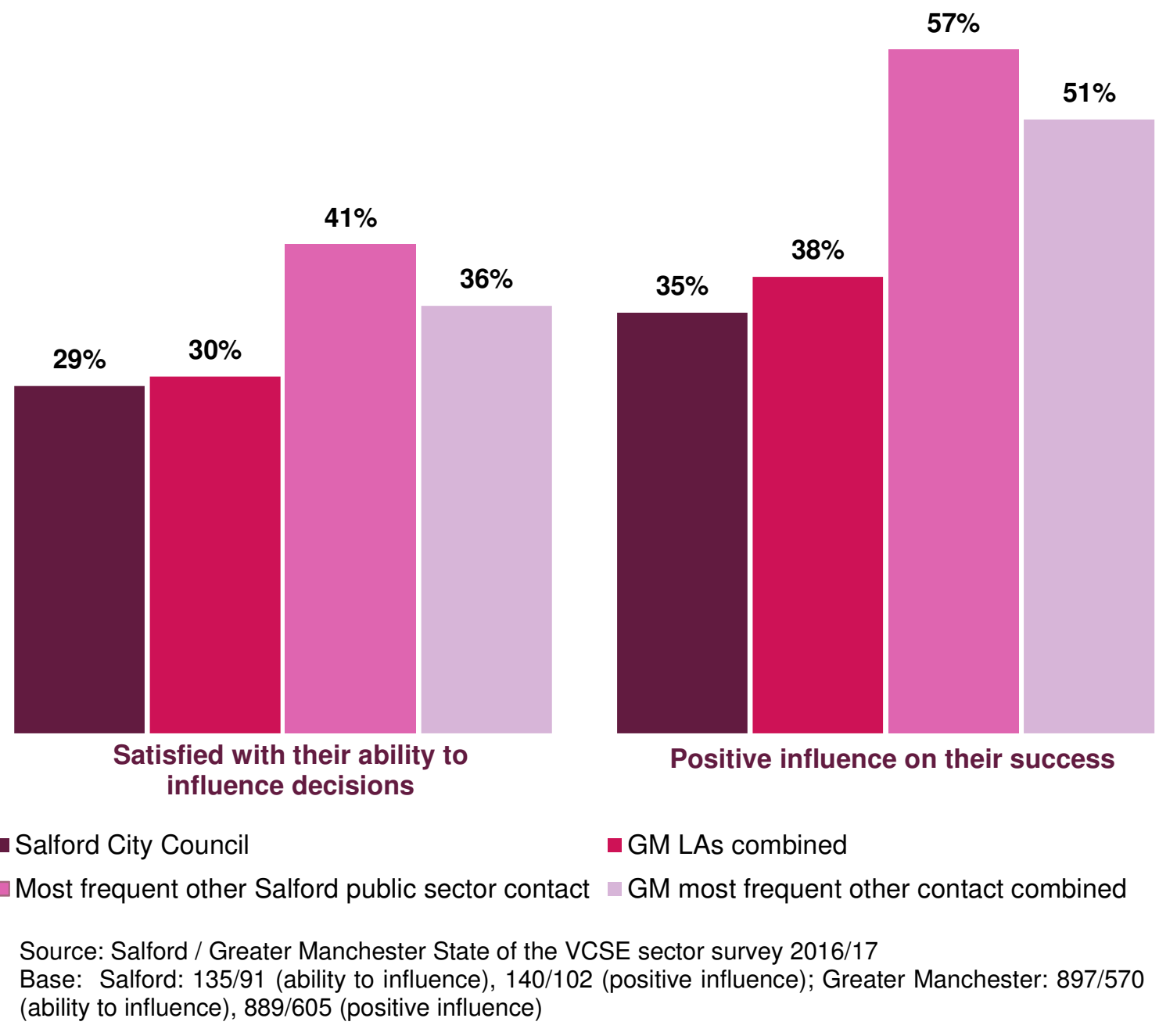

\subsection{Funding from local public sector bodies}

Respondents were also asked to reflect on their experiences of public sector funding in terms of how successful they had been, how satisfied they were with bidding arrangements, and how satisfied they were with the level of opportunity to bid for long-term funding.

Figure 8.5 shows responses to the question which asked organisations to consider how successful they had been in applying for funding or bidding for contracts. Results are split between perceptions of Salford City Council and of other public sector bodies. A comparison with the Greater Manchester average is also provided.

This shows that 42 per cent of respondents were successful in bidding for funding or contracts with Salford City Council compared to a 48 per cent success-rate with other public sector bodies. At the Greater Manchester level, a similar proportion (45 per cent) had been successful in bidding for funding or contracts from their local authority, but the figure was lower for other public sector bodies (40 per cent).

In 2012/13 a higher proportion indicated they had been successful in bidding for funding or contracts from Salford City Council (53 per cent) but the figure for other public sector bodies was lower (43 per cent). 
Figure 8.5: Success bidding for funding and contracts

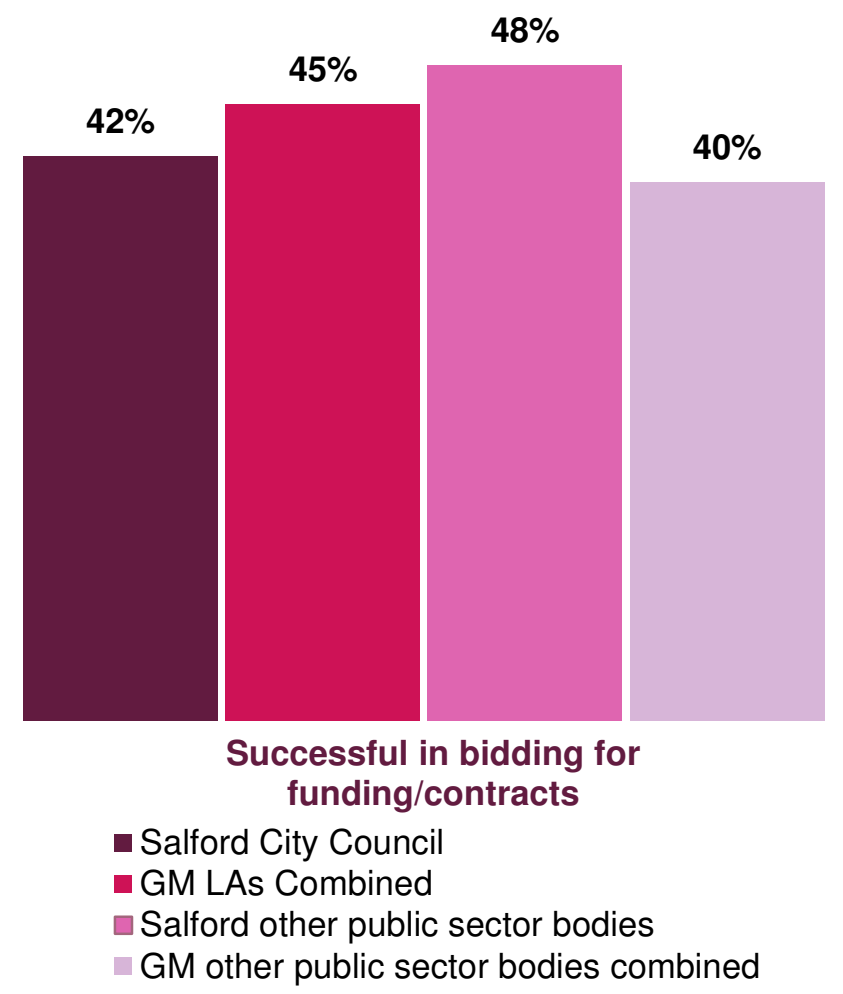

Source: Salford / Greater Manchester State of the VCSE sector survey 2016/17 Base: Salford: 178/170; Greater Manchester: 1,060/1,036

Respondents were asked specifically about Salford City Council and how satisfied they were with their grant funding and contract bidding arrangements and opportunities for funding and contracts lasting three years or longer. The responses are illustrated in figure 8.6. A comparison with the Greater Manchester local authority average is also provided.

Almost two-fifths (37 per cent) were satisfied with grant funding arrangements. Satisfaction with contract bidding arrangements was slightly lower at 31 per cent. Satisfaction with opportunities for both funding and contracts lasting three years or longer was lower (both below 20 per cent). The pattern was similar among the Greater Manchester combined figures. In 2012/13 respondents were not asked separately about grant funding and contracts. Just over one third (36 per cent) of respondents were satisfied with Salford City Council's funding/bidding arrangements in 2012/13 and 16 per cent were satisfied with their opportunities for funding/contracts which lasted three years or longer. 
Figure 8.6: Experiences of bidding for funding and contracts with local authorities

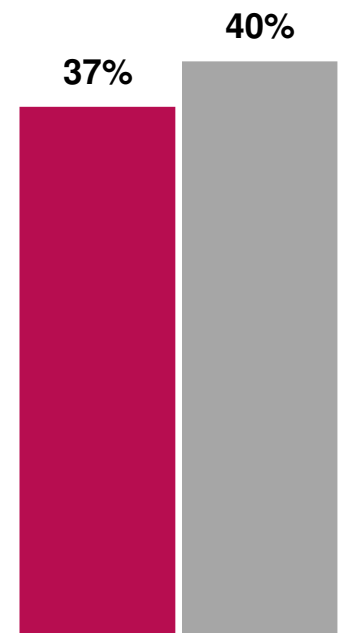

Satisfied with grant funding arrangements

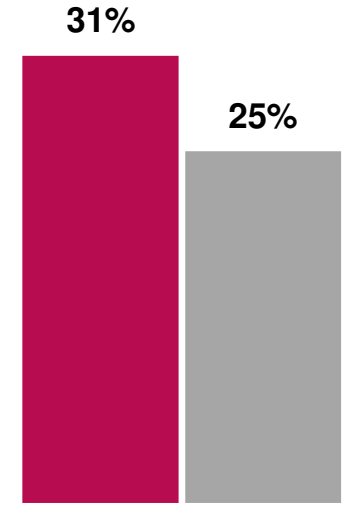

Satisfied with contract bidding arrangements

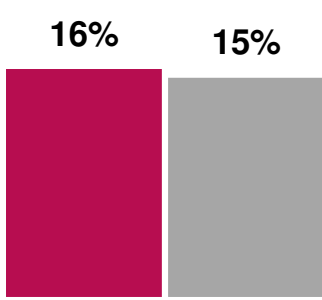

Satisfied with opportunities for funding that lasts 3yrs+ contracts that last 3yrs+

\section{- Salford City Council $\quad$ GM LAs Combined}

Source: Salford / Greater Manchester State of the VCSE sector survey 2016/17

Base: Salford: 116 (grant funding arrangements), 99 (contract bidding arrangements), 106 (opportunities for funding), 94 (opportunities for contracts); Greater Manchester: 808 (grant funding arrangements), 631 (contract bidding arrangements), 703 (opportunities for funding), 605 (opportunities for contracts)

Survey respondents were asked to consider how satisfied they were with the grant funding and contract bidding arrangements of their most frequent other public sector contact. As figure 8.7 shows, one third indicated they were satisfied, higher than across Greater Manchester as a whole (27 per cent).

They were also asked about their satisfaction with opportunities for funding and contracts longer than three years. Just 12 per cent were satisfied, almost identical to the Greater Manchester combined figure (11 per cent). 
Figure 8.7: Experiences of bidding for funding and contracts with other public sector bodies

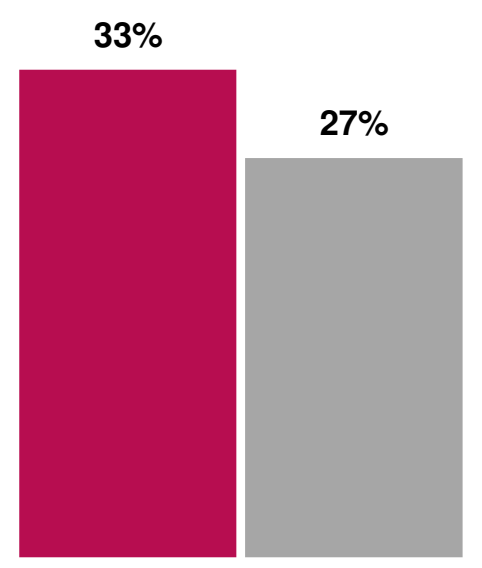

Satisfied with grant funding / contract bidding arrangements

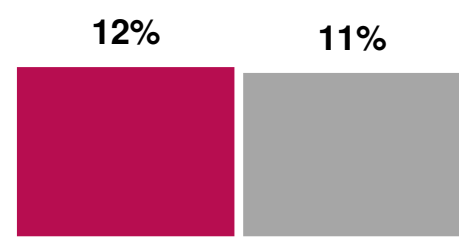

Satisfied with opportunities for funding / contracts that last 3yrs+

- Most frequent other Salford public sector contact

- GM other most frequent contact combined

Source: Salford / Greater Manchester State of the VCSE sector survey 2016/17

Base: Salford: 118 (funding/bidding arrangements), 113 (opportunities for funding/contracts); Greater Manchester: 705 (funding/bidding arrangements), 687 (opportunities for funding/contracts)

\subsection{Qualitative responses on relationships between the VCSE sector and local public sector bodies}

The focus groups discussed participants' views about and experiences of working with public sector bodies in Salford and more widely. Two key issues dominated these discussions: the prospects of devolution for VCSE organisations, and issues associated with funding from the public sector.

On devolution, there was widespread uncertainty amongst participants about what it would mean for VCSE organisations in the city. They were all aware of it, but said they had limited information on what it involved or how it would impact on all types of VCSE organisations.

"I think it will have an impact, but I don't know what the consequences will be for the sector."

"I have been to a couple of conferences and meetings and I think it will have a very big impact."

Overwhelmingly, participants did not view devolution as a positive development for their organisations or the community. They believed that the VCSEs were not seen as a key part of devolution plans, their voice would not be heard during discussions, and expressed their frustration with the apparent inability of Salford's VCSE sector to meaningfully influence devolution policy-development and decision-making.

"Yeah, the voluntary sector will be the last to know probably. I don't think that the sector is considered a key partner in the health and social care devolution." 
Overall, participants felt that the VCSE sector in Salford was better prepared than other areas for devolution due to the strong relationships and collaborative culture across the city in the last 30 years. They also felt that the amount of re-organising and re-evaluating across Greater Manchester would result in a huge shift towards the unknown, and that this was more likely to benefit the largest, well established Manchester-based charities.

"You have to sit in various meetings and groups and such, but I don't think we are truly influencing things, although Salford is in a better position than other Manchester areas."

"I'm concerned with how and where the funding will be given though. It could be a case of giving the power but not giving enough money and potentially I'm afraid that big, Greater Manchester charities will again run in and get the funding to fill some gaps, while other voluntary and community organisations miss out."

On the issue of public sector funding and their relationship with specific public sector bodies, VCSEs of all types highlighted that, after years of cuts, there was now very limited funding available from local public sector bodies. This meant many organisations now struggled to sustain key services and activities even though demand was reportedly rising. Furthermore, participants highlighted the bureaucratic maze and complexity involved in bidding for, allocating and using the funding that is available.

"Yes, for us too, now just about $10 \%$ of our income comes from the public sector, while in past years it was more than $80 \%$; the cuts have been massive."

"Yeah, if you don't tick the right box, or fill in the right information you don't get the funding. Also, there's this whole new jargon you have to get used to; it's so bloody hard to speak to people at times."

However, a number of participants highlighted the potential of greater funding from health bodies, whereas in the past the local authority had been the main source of funding.

"In the last 3 years I have very little funding from local authorities, but more recently we have created relationships with health and the CCG; but that has been a battle to be honest, a real battle. We purposefully changed our focus to be more in line with CCGs because they can commission differently."

One participant illustrated this point through an example in which the CCG paid a hospital, to pay a voluntary group, to develop a service. A further challenge was public bodies apparent unwillingness to fund long-term projects, favouring short term interventions instead.

On a more positive note, a number of participants expressed the view that Salford public sector bodies were much more accessible and open than other areas in Greater Manchester, and stressed the importance of trust-based personal relationships for determining the quality of their dealings with a public body at any given time. When it came to engaging and working with individuals in the public sector, participants suggested that, in recent years, many of the public agencies with which they had established good working relationships now had fewer officers with strong knowledge and experience of the VCSE sector, acknowledging the effects of public funding cuts and on public sector employment levels. It was mentioned that often, when the person whose input you trusted and whom you relied upon to be available to contact is removed (or retires), the associated project can collapse or be seriously undermined. 
"We work quite a lot with the Salford City College, they are very open about working with the voluntary sector but it again depends on specific people; it can vary."

"Access is good in comparison to Manchester. Here we can at least have a conversation; Salford is really special in that regard"

"With the CCG is also good although, you have to work a bit more; it's still learning."

"We work with probation quite consistently and I find them really open in Salford." 


\section{Partnership Working: the Private Sector}

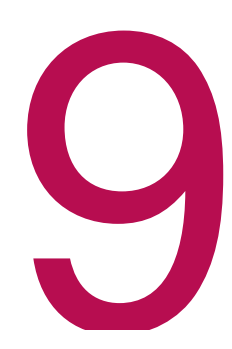

The previous chapter explored respondents' experiences of partnership working with public sector bodies. This chapter moves on to explore their experiences of working with private sector organisations. Only 19 per cent of survey respondents received any income through business donations. While this is an increase since the 2012/13 survey when just 13 per cent received this type of income, this area still appears to be new territory for many VCSE organisations. Survey respondents were asked about their direct dealings and experiences of working with private businesses in Salford.

\section{1. $\quad$ Working with private businesses}

Survey respondents were asked to indicate the extent to which they had direct dealings with private businesses in Salford. 55 per cent reported that they had some direct dealings, with 20 per cent having a 'great' or 'fair' amount of contact (figure 9.1). This is almost identical to the average for Greater Manchester as a whole (21 per cent 'great' or 'fair' amount of contact) and very similar to the 2012/13 survey where 55 per cent of respondents also reported some direct dealings, including 23 per cent having a 'great' or 'fair' amount of contact.

Figure 9.1: Extent of direct dealings with private businesses

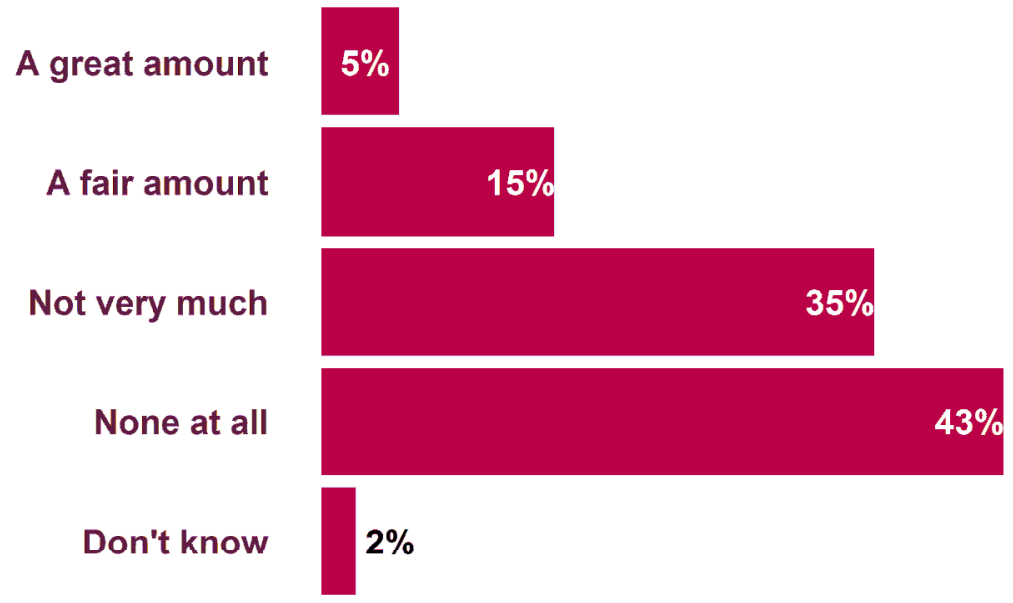

Source: Salford State of the VCSE sector survey 2016/17

Base: 183 
Just two per cent of organisations indicated they are members of a formal private sector-led consortium (the same as the Greater Manchester average), while nine per cent of respondents said their organisation is in formal partnership with any private sector organisations, slightly higher than the seven per cent of organisations across Greater Manchester.

Respondents were asked to comment on the influence private businesses have on their organisation's success. Taking all things into account, one third (33 per cent) of survey respondents felt that the private business community in Salford was a positive influence on their organisation's success. This is similar to the proportion for Greater Manchester as a whole (31 per cent) and an increase since the 2012/13 survey when just 21 per cent of survey respondents felt that the private business community in Salford was a positive influence on their organisation's success.

Figure 9.2: Private business community's influence on VCSE sector organisations' success

$$
\begin{array}{c|c}
\text { Very negative influence } & 1 \% \\
\text { Negative influence } & 4 \%
\end{array}
$$

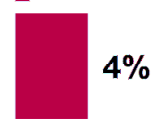

Neither positive nor negative influence

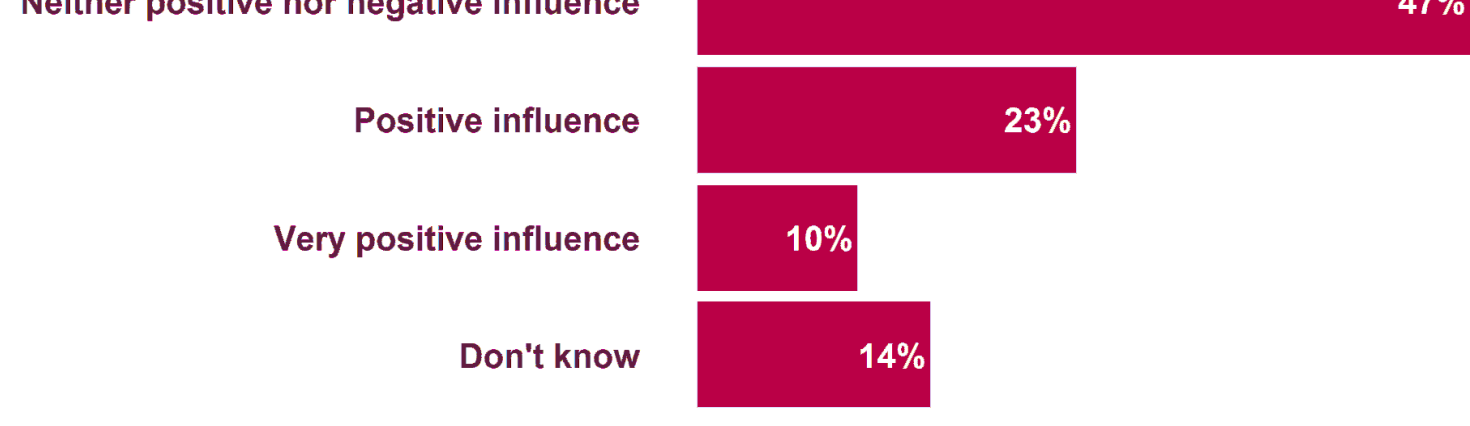

Source: Salford State of the VCSE sector survey 2016/17

Base: 112

\subsection{Qualitative responses on working with private sector businesses}

Focus group participants discussed their views about and experiences of working with private businesses. Overall, participants were positive about working with the private business sector and several mentioned that significant proportions of their income came from these collaborations and relationships. However, these relationships were often 'passive' - characterised by donations from local businesses

- rather than 'active' support or engagement in their work.

"We were put into contact with this big business and they appeared on Christmas Eve and brought us hundreds of toys and they asked 'will you distribute them?' So it's good, but there are bumps because business is always business and they fail to see the actual work we are doing and the actual issues in the community we try to address."

"It's very important for us and it is almost $70 \%$ of our income. Donating items and trading services even on sustainable businesses for example." 
A common reflection was that long-term, more formal collaborations with the private sector were difficult to achieve and would not necessarily work due to the 'cultural gap' that exists between the two sectors. Despite this gap some participants felt it was important for VCSEs to make the effort to understand the language and outlook of businesses and develop stronger, long-standing relationships with the private sector, especially locally. Through such collaborations it was hoped that VCSE organisations could raise awareness of social issues within businesses, and maybe change the way they worked.

"It's a huge shift in the culture though, because you have to understand their language and your staff must adapt quickly to that, but if you build some trust with them, we can be very productive."

"It is challenging to approach and communicate with them sometimes though. Having the confidence and the skills to do it well is not easy. So what we are trying to do, is to lay a foundation for the future of the organisation through these contacts with the corporate sector."

Participants with the most positive experiences of working with the private sector reported that, once a relationship had been established with a private business, things can move along in a much more straightforward and smooth manner in comparison to the public sector.

"Once you got the relationship it can be more stable than what you have with a public sector agency; it's more direct."

"Yeah, it can vary really, but generally I agree; it's more straightforward and we, as a sector, could involve them in the community; even if just by communicating with them and maybe organising events."

The challenge, they argued, was to build a foundation of trust with the appropriate person in a business, who had a sincere desire and the openness to learn, and understand what the VCSE sector does and how it impacts the community. 


\section{Partnership Working: Voluntary Community and Social Enterprise Organisations}

The previous two chapters have explored respondents' experiences of working with organisations from the public and private sectors. This chapter discusses survey respondents' views on their work with other VCSE sector organisations.

\subsection{Working with other VCSE organisations}

Survey respondents were asked about the extent to which they had direct dealings with other VCSE sector organisations in both Salford and Greater Manchester.

The vast majority (91 per cent) had some direct dealings with other VCSE sector organisations in Salford, and as figure 10.1 illustrates, 67 per cent had a 'great' or 'fair amount' of contact. Results are almost identical, both across Greater Manchester, where 90 per cent had some direct dealings with other VCSE sector organisations in their local area and 67 per cent had a 'great' or 'fair amount' of contact, and also when compared to the 2012/13 survey, when 89 per cent had some direct dealings with other VCSE organisations in Salford and 66 per cent had a 'great' or 'fair amount' of contact.

The proportion of respondents reporting they had direct dealings with other VCSE sector organisations in Greater Manchester was lower (69 per cent), along with the proportion who had a 'great' or 'fair amount' of contact ( 40 per cent). Results were similar at the Greater Manchester level (70 per cent direct dealings and 37 per cent with a 'great' or 'fair amount' of contact). Survey respondents were only asked about their dealings with other VCSE organisations across Greater Manchester in 2016/17. 
Figure 10.1: Extent of direct dealings with VCSE organisations

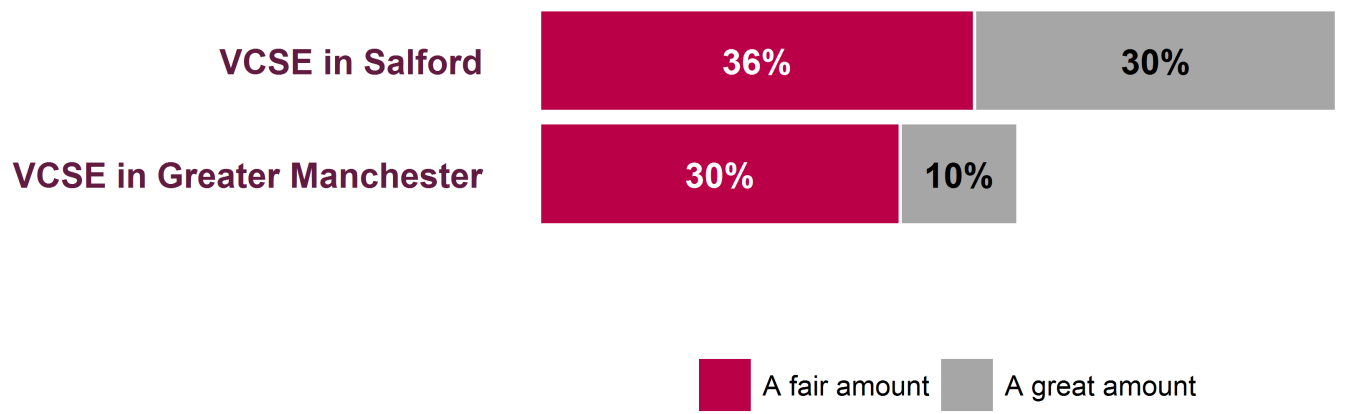

Source: Salford State of the VCSE sector survey 2016/17

Base: 181 (Salford) / 162 (Greater Manchester)

Respondents were asked to reflect on the opportunities they had to work with other VCSE sector organisations in terms of influencing local decisions, delivering local services and networking. Figure 10.2 summarises the responses.

Figure 10.2: Satisfaction with opportunities to work with VCSE organisations

Satisfied with opportunities
to work together to influence
decisions

Satisfied with opportunities to work together to deliver local services

Satisfied with opportunities to network with other VCSE organisations
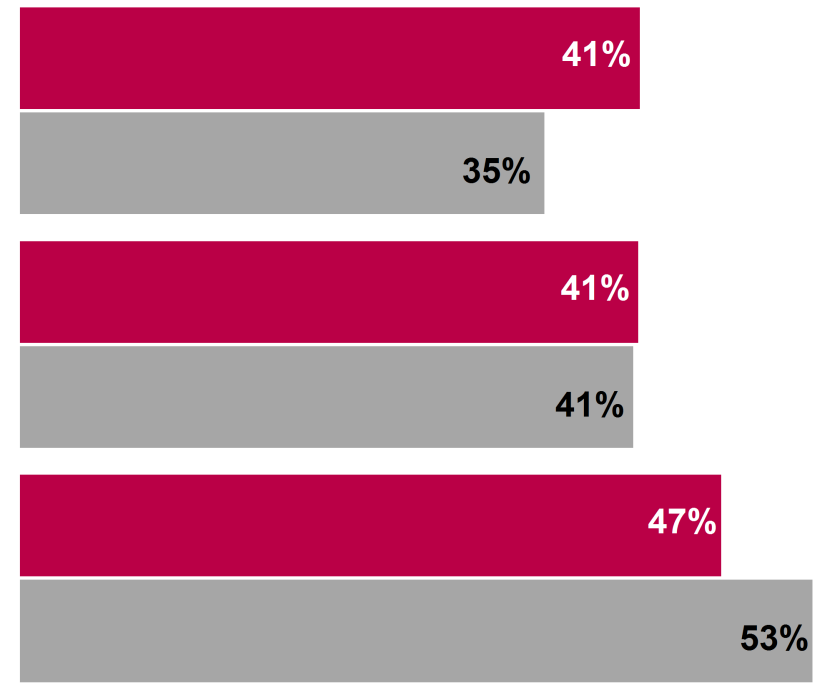

Salford GM Comparison

Source: Salford State of the VCSE sector survey 2016/17

Base: 180 (influence decisions) / 176 (delivering services) / 178 (networking)

This shows that 35 per cent of respondents were satisfied with the availability of opportunities to influence local decisions (47 per cent in 2012/13) and that 41 per cent were satisfied with the availability of opportunities to work together to deliver local services (46 per cent in 2012/13). A higher proportion of organisations across Greater Manchester were satisfied with opportunities to influence local decisions (41 per cent), however, the proportion satisfied with opportunities to work together to deliver local services was identical (41 per cent). Over half (53 per cent) of respondents were also satisfied with opportunities to network with other VCSE organisations (47 per cent across Greater Manchester as a whole). This was a new question added to the latest survey. 
Over one quarter (28 per cent) of respondents said their organisation is a member of a formal VCSE sector consortium, slightly higher than the 22 per cent of organisations across Greater Manchester. The 'Salford Third Sector Consortium' was the most common response received when respondents were asked to specify which consortium they were a member of.

Almost one fifth (19 per cent) also indicated their organisation is in another type of formal partnership with other VCSE organisations to deliver specific services (13 per cent across Greater Manchester). A wide range of responses were received when organisations were asked to specify which partnership they were members of, with a range of services covered by partnerships.

\subsection{Qualitative reflections on working with other VCSE organisations}

The focus groups discussed participants' views about and experiences of working in partnership with other VCSE organisations in Salford and more widely. Overall, participants were positive about working with other similar organisations. They felt that the VCSE sector in Salford was characterised by a strong cooperative culture and as such, respondents reported collaborating on various levels with other VCSEs, especially locally, with Salford CVS highlighted as an important facilitator of this activity.

"It has been a brilliant experience working with other organisations especially locally, co-organising events, it's been great. It's important to have strong, positive relationships with other VCSEs."

"I think one of the main things is that CVS is now the nucleus of the third sector in Salford and the ability to meet up regularly is so important; and if these relationships hadn't been facilitated by CVS, we wouldn't have these contacts. So I think it's absolutely imperative to try and cooperate."

Participants emphasised how effective partnerships depend very much on individual relationships, trust, and a 'good fit' between the involved organisations' identities, aims and abilities.

"We can't disrespect and disregard anyone and anything they are doing in the sector, but I genuinely feel that I will partner only with those with whom I have a professional connection and can do the work properly."

Larger charities in particular reported that they had been involved in a lot more partnership funding bids recently but that a downside of this had been fewer opportunities to work with small voluntary and community groups as they had in the past. This was mirrored by the views of smaller voluntary organisations who consistently reported they had insufficient resources to engage extensively in partnership working, and that they needed to focus on keeping their own services going.

"There are some really fantastic local partnerships but sometimes, because lots of groups have such limited capacity in what they are doing they are saying: 'we know you do that better than us, so we don't want to expose ourselves'. So for us, the partnerships that we have, are a result of painstaking financial, administrative and personal relationships development. It takes a lot of time and effort."

Participants made particular mention of the annual voluntary sector conference, which apart from bringing organisations together, helped build collective confidence, by highlighting how much had been accomplished in the VCSE sector and the area in the last 30 years. 
Generally, participants felt that there was much more that could be done in terms of partnership working so that VCSE organisations could pool their strengths together, sharing information and resources. The funding problems that the sector faced, however, had really 'stretched' organisations, forcing them to become more preoccupied with their survival, rather than partnerships, at least in the short term.

"For me it's funding and financial incentive. Sometimes bigger organisations are far more attractive to get funds, so they resist partners and I don't want to be the tick-box for them to get funding; I want to be an organic partner in a collaborative effort." 


\section{The Future}

This chapter details the responses received to questions about the future in the survey of organisations.

\subsection{Factors assisting or constraining delivery}

Respondents were asked to consider the factors they anticipated assisting or constraining their organisation over the next 12 months. Figure 11.1 illustrates that over half of respondents thought the following factors would assist their organisation over the next year:

- engagement with other VCSE organisations: 53 per cent anticipated this factor assisting their organisation; including 12 per cent who saw this as 'greatly assisting' and 42 per cent 'assisting'

- ability to retain staff/staff turnover: 53 per cent anticipated this factor assisting their organisation; including 28 per cent who saw this as 'greatly assisting' and 25 per cent 'assisting'

- ability to retain volunteers/volunteer turnover: 51 per cent anticipated this factor assisting their organisation; including 22 per cent who saw this as 'greatly assisting' and 29 per cent 'assisting'.

Engagement with other VCSE organisations was also the most common factor selected across Greater Manchester, with 50 per cent of organisations envisaging this factor assisting their organisation over the next 12 months.

In contrast almost one third or more saw the following factors as constraining their organisation over the next 12 months:

- the local economy: 40 per cent anticipated this factor constraining their organisation; including 14 per cent who saw this as 'seriously constraining' and 26 per cent 'constraining'

- ability to secure contracts from public sector bodies: 32 per cent anticipated this factor constraining their organisation; including 18 per cent who saw this as 'seriously constraining' and 14 per cent 'constraining'.

The local economy was also the most common factor selected across Greater Manchester as a whole, with 38 per cent anticipating this factor constraining their organisation over the following year. 
Figure 11.1: Factors anticipated as assisting or constraining organisations over the next 12 months

\section{Engagement with other VCSE organisations}

\section{Ability to retain staff / staff turnover}

Ability to retain volunteers / volunteer turnover

Ability to recruit volunteers with sufficient skills

Ability to secure other sources of incomes Engagement with public
sector bodies

Ability to employ staff with sufficient skills

Ability to secure grant funding from public sector bodies

Engagement with the private sector

Ability to secure contracts from public sector bodies

The local economy
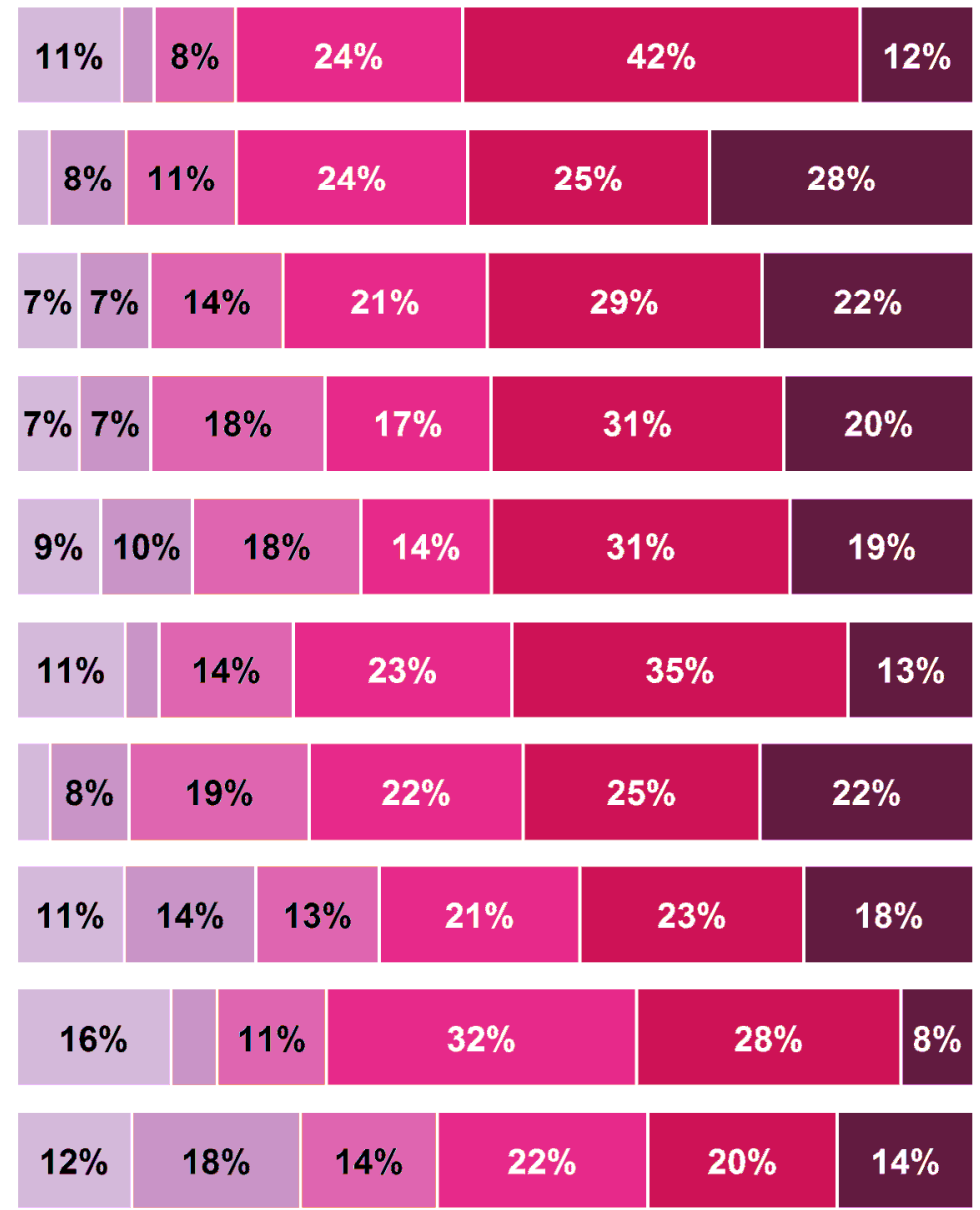

\begin{tabular}{|l|l|l|l|l|l|}
\hline $16 \%$ & $14 \%$ & $26 \%$ & $22 \%$ & $16 \%$ & $6 \%$ \\
\hline
\end{tabular}

$$
\text { Don't know } \quad \begin{aligned}
& \begin{array}{l}
\text { Seriously } \\
\text { constraining }
\end{array} \quad \text { Constraining } \quad \text { Neutral } \quad \text { Assisting } \\
& \text { assisting }
\end{aligned}
$$

Source: Salford State of the VCSE sector survey 2016/17

Base: $85-125$

Following on from quantitative questions regarding the factors organisations anticipated assisting or constraining their organisation over the next year, respondents were also asked to provide further qualitative (i.e. written) information about these factors.

Most comments focused on the factors constraining organisations, with issues accessing funding and recruiting and retaining volunteers and staff with appropriate skills common themes in the responses:

"A struggle to keep income at previous levels, we are restructuring our services around anticipated reduction in funding over next 3 years. Charging for services previously provided free of charge, difficult as we work with mostly unemployed adults with disabilities."

"It's getting more and more difficult to obtain funding as grants are usually given to new projects rather than existing ones. Larger organisations are taking over work 
done by charities like ours with higher costs so in effect the public sector are paying more for less."

"It's becoming more and more challenging to recruit and retain volunteers; more public health funding opportunities are required; there is an increased need to generate our own income/fundraise as there are less and less grant funding/ suitable contract opportunities available; alongside demand rising i.e.: more and more families needing our support due to the poor local economy / benefit reforms."

"We are struggling to get staff trained up to help grow the business and with no grants available and volunteers being few and far between these days it is getting harder to bring people into the business."

"Cuts in local authority and NHS funding, Increase in costs due to rise in living wage but not getting sufficient increase in cost from purchasers, difficulty in employing suitable staff due to low wage and high level of responsibility."

There were however some more positive comments received which focused on both existing arrangements and future strategies:

"Partnership work also plays a large part in our future and we are always looking to secure new and long lasting relationships with other VCSE organisations to build upon our skills and experience, share ideas and strengthen our future."

"Now we have our printer we can publicise more to hopefully increase our volunteers and we try to retain the one we have by providing travel expenses for them and generally keeping them happy by hopefully being a positive influence in their lives. We plan to ask for more help from public and private sector companies within the next year."

"Private groups and trusts continue to be a major source of funding along with the Salford CVS. We have excellent relationship with all of our supporters and have purposefully reduced our reserves to a more reasonable level."

"We anticipate that with the model and systems developed over the last 2 years we will be in a strong position to further develop our organisation"

\subsection{Current and future strategies}

Survey respondents were asked what strategies they are actively pursuing or planning to pursue. Figure 11.2 summarises the responses received and shows that over half of respondents were already doing or planning to do the following:

- working more closely with another voluntary/not-for-profit organisation: 59 per cent were already pursuing or planning to pursue this strategy; including 29 per cent who are doing this now and 30 per cent who are planning to do this in the future

- $\quad$ increasing earned income: 57 per cent were already pursuing or planning to pursue this strategy; including 25 per cent who are doing this now and 31 per cent who are planning to do this

- changing the way you run your services or activities: 52 per cent were already pursuing or planning to pursue this strategy; including 22 per cent who are doing this now and 30 per cent who are planning to do this. 
Results across Greater Manchester followed the same pattern, with the same ordering of strategies being pursued or planning to be pursued.

\section{Figure 11.2: Strategies being planned or pursued}

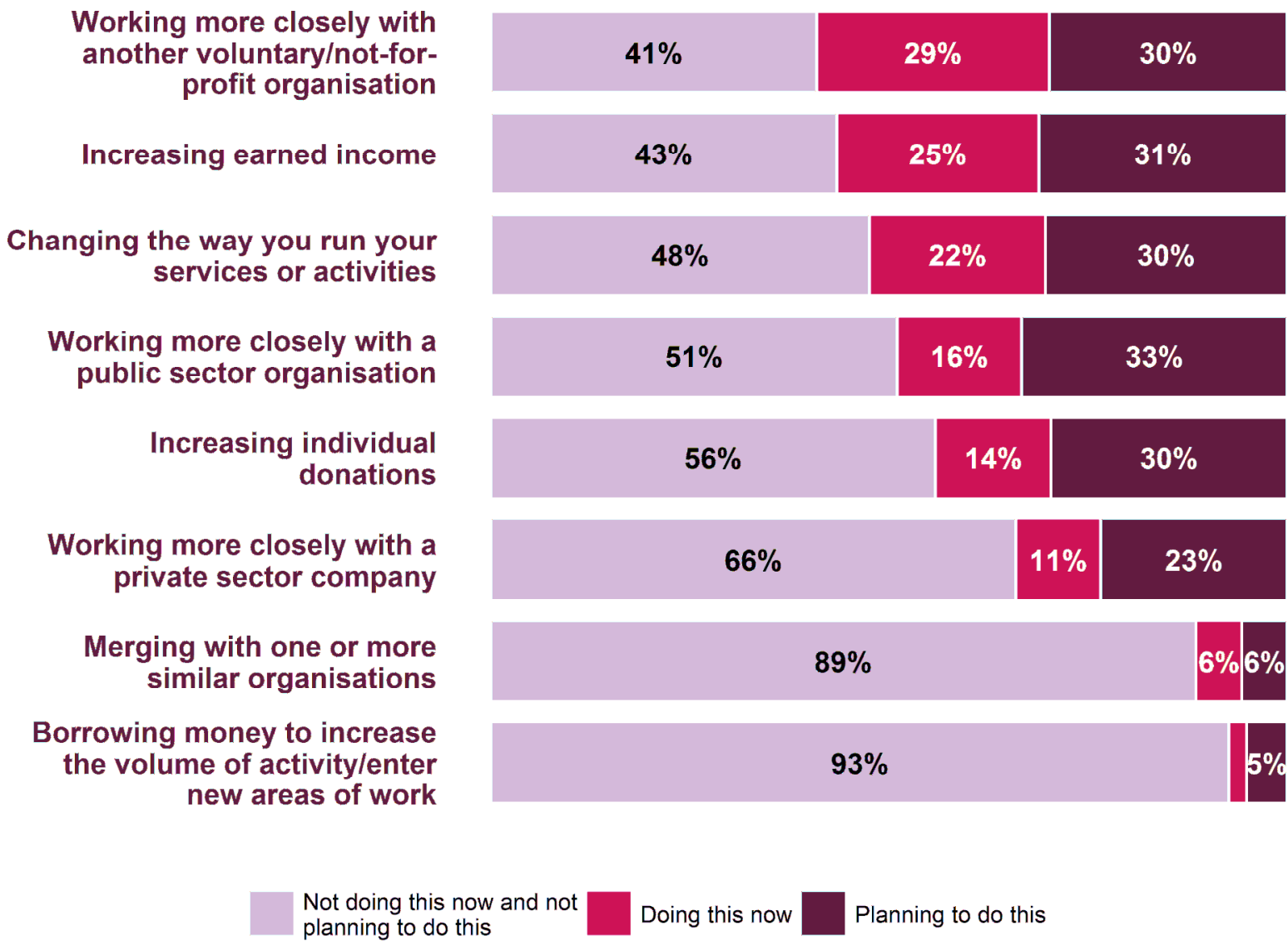

Source: Salford State of the VCSE sector survey 2016/17

Base: 137-145 


\section{Conclusions}

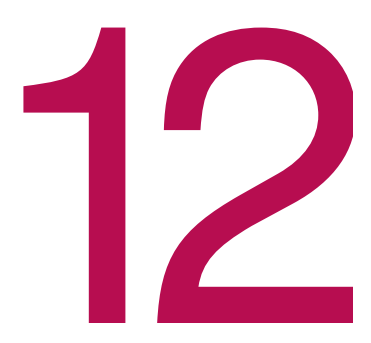

\section{The VCSE sector in Salford continues to occupy an important strategic position between policy development, service provision and everyday life.}

There are an estimated 1,513 organisations working in the VCSE sector in Salford who are involved in many areas of activity.

As in the 2013 study, the local area is a main focus for the majority of organisations; 35 per cent identified particular neighbourhoods or communities in Salford as their highest main geographic focus and a further 26 per cent identified the whole Salford local authority area as their highest main geographic focus.

The thematic areas with the greatest proportion of organisations working in them are: health and wellbeing; community development; sport and leisure; and education, training and research; the same four areas selected most frequently in 2012/13.

The VCSE sector plays a key role in fostering strong and cohesive communities within Salford and is an essential part of the social fabric of the City. Over threequarters (77 per cent) of organisations felt they are improving people's mental wellbeing while two-thirds (65 per cent) stated they are increasing people's skills.

2. The sector in Salford remains an important economic player, contributing significantly to GVA $^{27}$, but patterns in income, expenditure and the level of reserves suggest that, as in 2013, the sustainability of many organisations may be under threat.

Total income of the VCSE sector in $2014 / 15$ is estimated to be $£ 165$ million. This represents an increase of three per cent compared to 2013/14 when the total income of the VCSE sector was an estimated $£ 160$ million. The vast majority of organisations are micro or small although the majority of income is concentrated in large and medium-sized organisations.

The picture is more positive overall than in the previous 2013 study which identified year-on-year reductions in income. However analysis of income data across Greater Manchester by organisation size revealed micro and small organisations experienced year on year reductions in total income between 2012/13 and 2014/15. By contrast medium and large organisations saw a reduction in total income between 2012/13 and 2013/14 but then an increase between 2013/14 and 2014/15.

Over half (56 per cent) of respondents reported increasing their expenditure but only 48 per cent had experienced an increase in income and only 27 per cent report an increase in reserves.

\footnotetext{
${ }^{27}$ Gross Value Added (GVA), the value of goods and services produced, is a key measure of the economic contribution of organisations or sectors.
} 
In addition, 24 per cent of respondents reported a decrease in income but only 14 per cent reduced their expenditure.

21 per cent of respondents provided an expenditure figure for 2014/15 that was greater than their income.

These results indicate a sizeable number of organisations spent more money than they received in the last 12 months and that a considerable number of organisations are using their reserves to supplement their income, potentially leaving them in a fragile financial position.

\section{The VCSE sector in Salford continues to provide significant social value.}

It is estimated that the VCSE sector in Salford made 2.4 million interventions with clients, users or beneficiaries in the previous year.

VCSE organisations work with a range of different people, especially older people and children and young people, but also people from vulnerable groups (for example those with health problems).

\section{The VCSE sector continues to be a significant employer.}

In 2016/17 there were an estimated 3,500 FTE paid staff. In addition the sector was supported by 36,500 volunteers and 10,400 committee/board members who combined donated 115,400 hours per week.

Valuing the contribution of paid employees to Salford organisations by the expected value of the output that they produced gives an estimated annual contribution of $£ 111.2$ million. Doing the same for volunteers and committee/board members gives an estimated contribution of $£ 104.4$ million.

5. Volunteering is a core and unique feature of the work of the VCSE sector in Salford, however there are challenges associated with volunteering across the City.

Volunteering was regarded by focus group participants as a core and unique feature of their work with a range of benefits for the organisations. Volunteers brought new skill-sets and fresh ideas; enabled new services to be developed and existing services to be improved; improved organisational democracy; increased the visibility of the organisation in the community; and raised diversity and cultural awareness, ensuring accountability and staff responsibility.

Almost two-fifths (39 per cent) of organisations responding to the survey reported increased numbers of volunteers compared to the previous year, while 12 per cent of organisations reported a decrease in volunteer numbers.

The focus groups provided a mixed picture of whether or not levels of volunteering had changed in recent years.

Focus group participants also identified a number of challenges associated with volunteering. In particular they consistently argued that recruiting volunteers had become a costly and demanding process: the number of volunteers is not the issue most of the time, but the 'quality' and 'type' of volunteers and the resources to support them. 


\section{There is a mixed picture in Salford regarding relationships between the VCSE sector and public sector bodies.}

Overall, 78 per cent of respondents in Salford had some dealings with Salford City Council (77 per cent in 2012/13): 14 per cent had a great amount of dealings with the Council and 32 per cent had a fair amount of dealings.

Over one quarter (29 per cent) of respondents were satisfied with their ability to influence Salford City Council decisions of relevance to their organisation while 35 per cent said Salford City Council had a positive influence on their organisation's success, both more positive than the proportions reported in 2013.

All types of VCSE organisations who participated in focus groups highlighted that, after years of cuts, there was now very limited funding available from local public sector bodies. There was also widespread uncertainty amongst participants about what devolution would mean for VCSE organisations in the City. Overwhelmingly, participants did not view devolution as a positive development for their organisations or the community.

On a more positive note, a number of focus group participants expressed the view that Salford public sector bodies were much more accessible and open than other areas in Greater Manchester, and stressed the importance of trust-based personal relationships for determining the quality of their dealings with a public body at any given time.

\section{Perceptions of the private business sector appear to have improved, however engagement is still relatively low.}

55 per cent of organisations had some direct dealings with private businesses, with 20 per cent having a 'great' or 'fair' amount of contact. This is similar to 2012/13 when 55 per cent reported some direct dealings, including 23 per cent having a 'great' or 'fair' amount of contact.

One third felt that the private business community in Salford was a positive influence on their organisation's success. This is an increase since 2012/13 when just 21 per cent agreed private businesses were a positive influence.

Overall, focus group participants were positive about working with the private business sector and several mentioned that significant proportions of their income came from these collaborations and relationships. However, these relationships were often 'passive' - characterised by donations from local businesses - rather than 'active' support or engagement in their work.

\section{The VCSE sector in Salford continues to be well connected internally although most contact appears to be informal.}

As in the 2013 study, the vast majority of organisations had some direct dealings with other VCSE sector organisations in their local area, including 67 per cent who had a 'great' or 'fair amount' of contact.

28 per cent of respondents said their organisation is a member of a formal VCSE sector consortium.

Overall, focus group participants were positive about working with other similar organisations. They felt that the VCSE sector in Salford was characterised by a strong cooperative culture and as such, respondents reported collaborating on various levels with other VCSEs, especially locally, with Salford CVS highlighted as an important facilitator of this activity. 
Generally, participants felt that there was much more that could be done in terms of partnership working, but, the funding problems that the sector faced had really 'stretched' organisations, forcing them to become more preoccupied with their survival, rather than partnerships, at least in the short term.

\section{The sector still faces an uncertain future.}

With austerity measures set to continue for the foreseeable future and public sector funding for the sector continuing to be squeezed, there are still reasons for caution within the sector.

Respondents appear to recognise this uncertainty and are pursuing a range of strategies to ensure their sustainability, in particular: generating earned income from other sources, partnership working and organisational change. 


\section{Appendix 1}

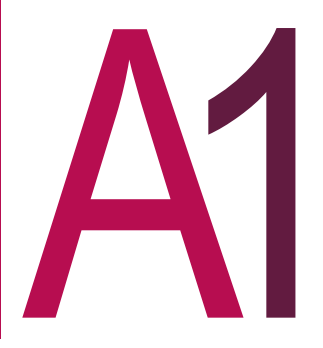

\section{Methodology}

\section{Survey of organisations}

At least partial responses were received from 54 of the 720 organisations that were sent a survey questionnaire: this represents a response rate of eight per cent. Another web-based version of the survey was also distributed by Salford CVS, reaching organisations also included in the original sample and beyond. Salford CVS played a key role in boosting the response rate to the survey by utilising their relationships within the sector to encourage organisations to complete a questionnaire. In addition GMCVO distributed a version of the survey via their networks. A further 144 responses were collected via this method, meaning a total of 198 responses were collected overall during September 2016 - January 2017, giving a higher overall response rate.

The survey was undertaken as part of a wider study in six other Greater Manchester boroughs: Bolton, the City of Manchester, Oldham, Rochdale, Stockport and Tameside.

The questionnaire was based on the one originally developed for the 'State of the Voluntary Sector Survey' undertaken in Salford in 2010. The questionnaire was revised for the 'Greater Manchester State of the Voluntary Sector' research undertaken in 2012/13 and again for this wave of the survey following input from the Research Steering Group. The Greater Manchester Chief Officers Group also provided additional oversight regarding the survey design and implementation.

The questionnaire provided data on various aspects of the VCSE sector including:

- the scale and scope of its activity, including the roles organisations undertake, the people they support, and the areas they benefit

- the economic impact of its work, including income and expenditure, sources of funding, the role of paid staff and volunteers, and financial sustainability

- relationships with the public sector, including Salford City Council, public sector health bodies, and a range of other local statutory bodies

- relationships with other local organisations, including VCSE organisations and private businesses.

Where possible the report compares results from the latest survey and the 2012/13 study. Revisions to the questionnaire mean that comparisons are not always possible or appropriate. It is also worth noting that in 2012/13 a large postal survey was the main method of data collection which was supplemented with a web based survey. This is different to the latest study when a web based survey was the primary method of data collection. 
When reading the report it is important to acknowledge two key points. First, the results reported are based on the survey responses received. Therefore it is possible that if a different sample of organisations had taken part in the survey different results may have emerged. It is estimated that the results reported are within $+/-6.5$ percentage points of the true value.

Secondly, on a number of occasions the analysis in this report has used extrapolations from the survey responses to provide estimates of totals for all organisations that work in the VCSE sector including:

- the number of clients, users and beneficiaries of the sector

- the total income of the sector

- $\quad$ and the number of FTE paid staff and the number of volunteers and committee/board members that are part of the sector's workforce; including the hours per week that volunteers contribute.

This was necessary to take account of noticeable differences in the response rates by organisation size. A failure to do this would lead to upwardly biased estimates: a small number of mainly 'large' organisations create a high mean value that is not representative of the majority of organisations. This is an important point given that we estimate that a large proportion of the sector is made up of 'micro' organisations which tend to have far lower values and not taking into account difference by size of organisations would produce estimates that are much higher.

Table A1: Extrapolations: a worked example (total annual income)

\begin{tabular}{lrrr}
\hline & $\begin{array}{c}\text { Average income by } \\
\text { size } \\
\text { (a) }\end{array}$ & $\begin{array}{c}\text { Estimated number } \\
\text { of organisations } \\
\text { (b) }\end{array}$ & $\begin{array}{c}\text { Total income } \\
\text { (thousands) } \\
\text { (c) }\end{array}$ \\
\hline Micro (under $£ 10 k$ ) & $£ 2,438$ & 1,038 & $£ 2,530,635$ \\
Small (£10k to $1100 k)$ & $£ 38,844$ & 271 & $£ 10,532,661$ \\
Medium (£100k to $11 \mathrm{~m})$ & $£ 320,581$ & 158 & $£ 50,607,463$ \\
Large (over $£ 1 \mathrm{~m})$ & $£ 2,201,023$ & 46 & $£ 101,078,278$ \\
\hline Total & & & (b) $£ 164,749,036$ \\
\hline
\end{tabular}

Please note it has been assumed here that the estimated averages for Greater Manchester organisations are representative for organisations within Salford. So for example it is has been assumed that the estimated average income of approximately $£ 320,600$ for medium sized organisations across Greater Manchester is representative of the income for medium sized organisations within Salford.

Using the Greater Manchester averages improves the reliability of the estimates.

\section{Focus groups}

To provide a further depth of understanding to some of the themes covered in the State of the Sector Survey, three focus groups were conducted. The focus groups were held midway through the survey administration and undertaken by Salford CVS who recruited local organisations to participate in the groups.

A topic guide was devised to help guide discussions and ensure a standardised approach across all local authority areas conducting focus groups. The topic guide was created in 
partnership between CRESR and the Research Steering Group with CRESR providing advice and guidance on best practice in undertaking this type of research.

The focus groups lasted approximately 1 hour - 1 hour 30 minutes and were digitally recorded where consent was obtained. The recordings were then provided to CRESR who analysed the discussions. Analysis of the discussions is included in the relevant chapters of this report.

The topics discussed in the focus groups concentrated on four key themes: volunteering, working with the public sector, working with other VCSE organisations and working with the private business sector.

Focus groups took place with organisations from across Salford, with participants selected by organisation type specifically registered charities, small VCSE groups and social enterprises.

\section{Legal status of responding organisations}

Respondents to the questionnaire were asked to identify the legal status of their organisation. For this question it was possible for organisations to select registered charity in addition to identifying their legal form. Figure A1 below shows that 40 per cent were a group with a constitution, but not registered charities and 21 per cent of organisations were a company limited by guarantee and that separate to identifying their legal status two fifths of respondents, 40 per cent, identified that their organisation was a registered charity.

These results are slightly different to the $2012 / 13$ survey when:

- 34 per cent of organisations were a group with a constitution, but not registered charities (25 per cent were an unincorporated associated group (constituted group in 2010))

- 29 per cent were companies limited by guarantee (26 per cent 2010)

- $\quad$ no organisations had no legally constituted form (also none in 2010)

- 55 per cent of respondents identified that their organisation was a registered charity (61 per cent in 2010).

Since the earlier study there has been a change in the legal forms available to VCSE organisations and this question was subsequently revised for the latest study. The changes above are likely to reflect these new opportunities. Charitable Incorporated Organisation and Community Benefit Society were additional options included in the latest version of the questionnaire.

Across Greater Manchester:

- 30 per cent of organisations were a group with a constitution, but not a registered charity

- 28 per cent were a company limited by guarantee

- four per cent of organisations had no legally constituted form

- 49 per cent of organisations were registered charities. 
Figure A1: The legal status of responding organisations

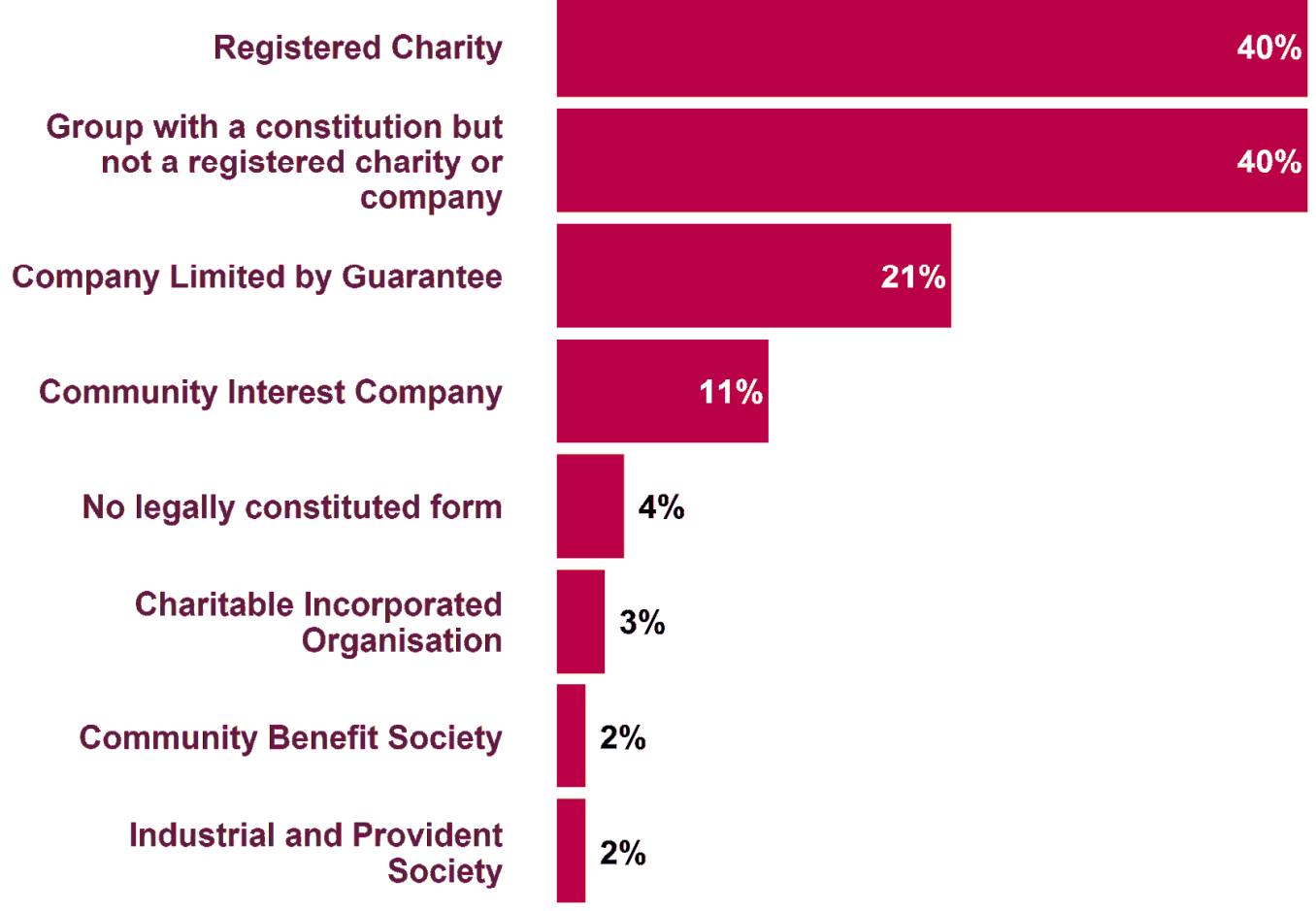

Source: Salford State of the VCSE sector survey 2016/17 Base: 195 


\section{Sheffield Hallam University}

Salford state of the voluntary, community and social enterprise sector 2017

DAMM, Christopher <http://orcid.org/0000-0002-7355-3496>, PRINOS, Ioannis and SANDERSON, Elizabeth <http://orcid.org/0000-0003-1423-1670>

Available from the Sheffield Hallam University Research Archive (SHURA) at:

http://shura.shu.ac.uk/16671/

\section{Copyright and re-use policy}

Please visit http://shura.shu.ac.uk/16671/ and http://shura.shu.ac.uk/information.html for further details about copyright and re-use permissions. 